\title{
Hypercontact structures and Floer homology
}

\author{
SONJA HOHLOCH \\ GREGOR NOETZEL \\ DIETMAR A SALAMON
}

\begin{abstract}
We introduce a new Floer theory associated to a pair consisting of a Cartan hypercontact 3-manifold $M$ and a hyperkähler manifold $X$. The theory is a based on the gradient flow of the hypersymplectic action functional on the space of maps from $M$ to $X$. The gradient flow lines satisfy a nonlinear analogue of the Dirac equation. We work out the details of the analysis and compute the Floer homology groups in the case where $X$ is flat. As a corollary we derive an existence theorem for the 3-dimensional perturbed nonlinear Dirac equation.
\end{abstract}

53D40, 32Q15

\section{Introduction}

In this paper we examine a hyperkähler analogue of symplectic Floer homology. We assume throughout that $X$ is a hyperkähler manifold with complex structures $I, J, K$ and symplectic forms $\omega_{1}, \omega_{2}, \omega_{3}$. We also assume that $M$ is a compact oriented 3manifold equipped with a volume form $\sigma \in \Omega^{3}(M)$ and a positive frame $v_{1}, v_{2}, v_{3} \in$ $\operatorname{Vect}(M)$ of the tangent bundle. Associated to these data is a natural $1-$ form on the space $\mathcal{F}:=C^{\infty}(M, X)$ of smooth functions $f: M \rightarrow X$ defined by

$$
\widehat{f} \mapsto \int_{M}\left(\omega_{1}\left(\partial_{v_{1}} f, \widehat{f}\right)+\omega_{2}\left(\partial_{v_{2}} f, \widehat{f}\right)+\omega_{3}\left(\partial_{v_{3}} f, \widehat{f}\right)\right) \sigma
$$

for $\hat{f} \in T_{f} \mathcal{F}=\Omega^{0}\left(M, f^{*} T X\right)$. This 1 -form is closed if and only if the vector fields $v_{i}$ are volume preserving, ie

$$
\mathcal{L}_{v_{1}} \sigma=\mathcal{L}_{v_{2}} \sigma=\mathcal{L}_{v_{3}} \sigma=0 .
$$

Since every closed oriented 3-manifold is parallelizable it admits a volume preserving frame (see Gromov [17, Section 2.4.3]). Our main examples are the 3-torus with the coordinate vector fields and the 3 -sphere with the standard hypercontact structure. 


\section{Hypercontact structures}

A hypercontact structure on a 3-manifold $M$ is a triple of contact forms $\alpha=$ $\left(\alpha_{1}, \alpha_{2}, \alpha_{3}\right) \in \Omega^{1}\left(M, \mathbb{R}^{3}\right)$ such that

$$
\alpha_{1} \wedge d \alpha_{1}=\alpha_{2} \wedge d \alpha_{2}=\alpha_{3} \wedge d \alpha_{3}=: \sigma
$$

and $\alpha_{i} \wedge d \alpha_{j}+\alpha_{j} \wedge d \alpha_{i}=0$ for $i \neq j$. The Reeb vector fields $v_{1}, v_{2}, v_{3}$ are pointwise linearly independent and preserve the volume form $\sigma$. The hypercontact structure is called positive if they form a positive frame of the tangent bundle. In this setting the 1 -form (1) is the differential of the action functional $\mathcal{A}: \mathcal{F} \rightarrow \mathbb{R}$ defined by

$$
\mathcal{A}(f):=-\int_{M}\left(\alpha_{1} \wedge f^{*} \omega_{1}+\alpha_{2} \wedge f^{*} \omega_{2}+\alpha_{3} \wedge f^{*} \omega_{3}\right) .
$$

A positive hypercontact structure is called a Cartan structure if the $\alpha_{i}$ form a dual frame of the cotangent bundle, ie $\alpha_{i}\left(v_{j}\right)=\delta_{i j}$. In the Cartan case $\kappa:=d \alpha_{1}\left(v_{2}, v_{3}\right)=$ $d \alpha_{2}\left(v_{3}, v_{1}\right)=d \alpha_{3}\left(v_{1}, v_{2}\right)$ is constant and $d \alpha_{i}=\kappa \alpha_{j} \wedge \alpha_{k}$ and $\left[v_{i}, v_{j}\right]=\kappa v_{k}$ for every cyclic permutation $i, j, k$ of $1,2,3$. (We use the sign convention of McDuff and Salamon [22] for the Lie bracket.)

The archetypal example is the 3-sphere $M=S^{3}$, understood as the unit quaternions, with $v_{1}(y)=\mathbf{i} y, v_{2}(y)=\mathbf{j} y, v_{3}(y)=\mathbf{k} y$. Hypercontact structures were introduced by Geiges-Gonzalo $[13 ; 14]$. They use the term taut contact sphere for what we call a hypercontact structure. They proved that every Cartan hypercontact 3-manifold is diffeomorphic to a quotient of the 3-sphere by the right action of a finite subgroup of $\operatorname{Sp}(1)$.

\section{Tori}

Let $M=\mathbb{T}^{3}=\mathbb{R}^{3} / \mathbb{Z}^{3}$ be the standard 3-torus equipped with the standard volume form $\sigma=d t_{1} \wedge d t_{2} \wedge d t_{3}$ and $v_{i}=\sum_{j=1}^{3} a_{i j} \partial_{j}$ where $A=\left(a_{i j}\right)_{i, j=1}^{3}$ is a nonsingular real $3 \times 3$ matrix. In this case the lift of the 1 -form (1) to the universal cover $\widetilde{\mathcal{F}}$ of $\mathcal{F}$ is the differential of the function

$$
\mathcal{A}=\sum_{i, j=1}^{3} a_{i j} \mathcal{A}_{i j}: \widetilde{\mathcal{F}} \rightarrow \mathbb{R}
$$

where $\mathcal{A}_{i j}(f)$ denotes the $\omega_{i}$-symplectic action of the loop $t_{j} \mapsto f(t)$, averaged over the remaining two variables $t_{k}, t_{\ell}$ with $k, \ell \neq j$. If $X$ is flat and $\mathcal{F}_{0} \subset \mathcal{F}$ denotes the space of contractible maps $f: \mathbb{T}^{3} \rightarrow X$ then $\mathcal{A}$ descends to $\mathcal{F}_{0}$. Explicitly, we have $\mathcal{A}_{i j}(f):=-\int_{0}^{1} \int_{0}^{1} \int_{\mathbb{D}} u_{t_{k}, t_{\ell}}^{*} \omega_{i} d t_{k} d t_{\ell}$ for $f \in \mathcal{F}_{0}$, where $u_{t_{k}, t_{\ell}}: \mathbb{D} \rightarrow X$ is a smooth family of maps satisfying $u_{t_{k}, t_{\ell}}\left(e^{2 \pi i t_{j}}\right)=f\left(t_{1}, t_{2}, t_{3}\right)$. 


\section{Hyperbolic spaces}

A third class of examples arises from unit tangent bundles of higher genus surfaces or equivalently from quotients of the group $\mathrm{G}:=\operatorname{PSL}(2 ; \mathbb{R})$. Let $\mathcal{H} \subset \mathbb{C}$ denote the upper half plane and $\mathcal{P}:=\left\{(z, \zeta) \in \mathbb{C}^{2}|\operatorname{Im}(z)=| \zeta \mid\right\}$ the unit tangent bundle of $\mathcal{H}$. The group $\mathrm{G}$ acts freely and transitively on $\mathcal{P}$ by

$$
g_{*}(z, \zeta):=\left(\frac{a z+b}{c z+d}, \frac{\zeta}{(c z+d)^{2}}\right), \quad g=:\left(\begin{array}{ll}
a & b \\
c & d
\end{array}\right) \in \operatorname{SL}(2 ; \mathbb{R}) .
$$

Now let $\Gamma \subset \operatorname{PSL}(2 ; \mathbb{R})$ be a discrete subgroup acting freely on $\mathcal{H}$ such that the quotient $\Sigma:=\Gamma \backslash \mathcal{H}$ is a closed Riemann surface. Then the 3 -manifold

$$
M:=\Gamma \backslash \mathrm{G}
$$

is diffeomorphic to the unit tangent bundle $T_{1} \Sigma=\Gamma \backslash \mathcal{P}$ via $[g] \mapsto\left[g_{*}(i, 1)\right]$. The group $\mathrm{G}$ carries a natural bi-invariant volume form $\sigma \in \Omega^{3}(\mathrm{G})$ given by

$$
\sigma(g \xi, g \eta, g \zeta):=\frac{1}{2} \operatorname{trace}([\xi, \eta] \zeta)
$$

for $\xi, \eta, \zeta \in \mathfrak{g}:=\operatorname{Lie}(\mathrm{G})=\mathfrak{s l}(2 ; \mathbb{R})$. This volume form descends to $M$ and is invariant under the right action of $\mathrm{G}$. Now consider the traceless matrices

$$
\xi_{1}:=\left(\begin{array}{rr}
1 & 0 \\
0 & -1
\end{array}\right), \quad \xi_{2}:=\left(\begin{array}{ll}
0 & 1 \\
1 & 0
\end{array}\right), \quad \xi_{3}:=\left(\begin{array}{rr}
0 & -1 \\
1 & 0
\end{array}\right) .
$$

The resulting vector fields $v_{i}(g):=g \xi_{i}$ on $\mathrm{G}$ are $\Gamma$-equivariant and preserve the volume form $\sigma$. Hence they descend to volume preserving vector fields on $M$ (still denoted by $v_{i}$ ) and so the 1 -form (1) is closed in this setting.

Note that $\sigma\left(v_{1}, v_{2}, v_{3}\right)=2$ and $d \pi\left(v_{3}\right)=0, d \pi\left(v_{1}\right)=i d \pi\left(v_{2}\right)$. The Lie brackets of the vector fields $v_{i}$ are given by

$$
\left[v_{2}, v_{3}\right]=-2 v_{1}, \quad\left[v_{3}, v_{1}\right]=-2 v_{2}, \quad\left[v_{1}, v_{2}\right]=2 v_{3}
$$

(because the $\xi_{i}$ act on $\mathrm{G}$ on the right). Hence, if $\alpha_{i} \in \Omega^{1}(M)$ denote the 1 -forms dual to the vector fields $v_{i}$, we have

$$
d \alpha_{1}=-2 \alpha_{2} \wedge \alpha_{3}, \quad d \alpha_{2}=-2 \alpha_{3} \wedge \alpha_{1}, \quad d \alpha_{3}=2 \alpha_{1} \wedge \alpha_{2} .
$$

This implies that the 1 -form (1) is the differential of the action functional

$$
\mathcal{A}(f):=\int_{M}\left(\alpha_{1} \wedge f^{*} \omega_{1}+\alpha_{2} \wedge f^{*} \omega_{2}-\alpha_{3} \wedge f^{*} \omega_{3}\right) .
$$


However, in this setting the energy identity (7) discussed below does not help in the compactness proof. This is the reason why we do not include the higher genus case in our discussion in the main part of this paper.

\section{Floer theory}

The zeros of the 1-form (1) are the solutions $f: M \rightarrow X$ of the nonlinear elliptic first order partial differential equation

$$
\partial(f):=I \partial_{v_{1}} f+J \partial_{v_{2}} f+K \partial_{v_{3}} f=0 .
$$

This is a nonlinear analogue of the Dirac equation first introduced by Taubes [29]. Obviously, the constant functions are solutions of (4). When $M=S^{3}$ other solutions arise from the composition of rational curves with suitable Hopf fibrations (see below). When $M=\mathbb{T}^{3}$ solutions can be obtained from elliptic curves. In the case $M=$ $\Gamma \backslash \mathrm{G}$ solutions arise from the composition of $K$-holomorphic curves $\Sigma \rightarrow X$ with $\pi: M \rightarrow \Sigma$. These examples are all homologically trivial, even though Hopf fibrations over holomorphic spheres in the K3 surface do represent nontrivial homotopy classes in $\pi_{3}$. A homologically nontrivial example with target manifold $X:=\mathbb{H} / \mathbb{Z}^{4}$ with its standard hyperkähler structure and domain $M:=\mathbb{T}^{3}=\mathbb{R}^{3} / \mathbb{Z}^{3}$ with vector fields $v_{i}=\partial / \partial t_{i}$ is given by $f(t):=t_{1}+\mathbf{i} t_{2}+(1+\mathbf{j}) t_{3}$.

In this paper we prove an existence result for the solutions of the perturbed nonlinear Dirac equation

$$
\partial_{H}(f):=I \partial_{v_{1}} f+J \partial_{v_{2}} f+K \partial_{v_{3}} f-\nabla H(f)=0 .
$$

Here $H: X \times M \rightarrow \mathbb{R}$ is a smooth function and we denote by $\nabla H(f)$ the gradient with respect to the first argument. The linearized operator for this equation is self adjoint and we call a solution $f: M \rightarrow X$ of (5) nondegenerate if the linearized operator is bijective. In the nondegenerate case, and when $X$ is flat, one can count the solutions with signs, however, it turns out that this count gives zero. Nevertheless we shall prove the following hyperkähler analogue of the Conley-Zehnder theorem confirming the Arnold conjecture for the torus [4]. In fact, in the torus case with $v_{1}=\partial / \partial t_{1}$ the solutions of (4) can be interpreted as the periodic orbits of a suitable infinite dimensional Hamiltonian system.

Theorem A Let $M$ be either a compact Cartan hypercontact 3-manifold (with Reeb vector fields $v_{i}$ ) or the 3-torus (with a constant frame $v_{i}$ ). Let $X$ be a compact flat hyperkähler manifold. Then the space of solutions of (5) is compact. Moreover, if the contractible solutions are all nondegenerate, then their number is bounded below by the sum of the $\mathbb{Z}_{2}-$ Betti numbers of $X$. In particular, Equation (5) has a contractible solution for every $H$. 
The proof of Theorem A is based on the observation that the solutions of (5) are the critical points of the perturbed hypersymplectic action functional $\mathcal{A}_{H}(f):=\mathcal{A}(f)-$ $\int_{M} H(f) \sigma$. As in symplectic Floer theory, this functional is unbounded above and below, and the Hessian has infinitely many positive and negative eigenvalues. Thus the standard techniques of Morse theory are not available for the study of the critical points. However, with appropriate modifications, the familiar techniques of Floer homology carry over to the present case, at least when $X$ is flat, and thus give rise to natural Floer homology groups for a pair $(M, X)$.

The Floer groups are determined by a chain complex that is generated by the solutions of (5). The boundary operator is determined by the finite energy solutions $u: \mathbb{R} \times M \rightarrow X$ of the negative gradient flow equation

$$
\partial_{s} u+I \partial_{v_{1}} u+J \partial_{v_{2}} u+K \partial_{v_{3}} u=\nabla H(u) .
$$

One of the key ingredients in the compactness proof is the energy identity

$$
\frac{1}{2} \int_{M}|d f|^{2}=\frac{1}{2} \int\left|I \partial_{v_{1}} f+J \partial_{v_{2}} f+K \partial_{v_{3}} f\right|^{2}-\int_{M} \sum_{i=1}^{3} \varepsilon_{i} \wedge f^{*} \omega_{i}
$$

for $f: M \rightarrow X$, where the $\varepsilon_{i} \in \Omega^{1}(M)$ are dual to the vector fields $v_{i}$. In the torus case these forms are closed and thus the last term in (7) is a topological invariant. In the Cartan hypercontact case this term is the hypersymplectic action $\mathcal{A}(f)$.

To compute the Floer homology groups we choose a Morse-Smale function $H: X \rightarrow \mathbb{R}$ and study the equation

$$
\partial_{s} u+\varepsilon^{-1}\left(I \partial_{v_{1}} u+J \partial_{v_{2}} u+K \partial_{v_{3}} u\right)=\nabla H(u)
$$

for small values of $\varepsilon$. The gradient lines of $H$ are solutions of this equation and we shall prove that, for $\varepsilon>0$ sufficiently small, there are no other contractible solutions. This implies that our Floer homology groups $\operatorname{HF}_{*}(M, X)$ are isomorphic to the singular homology $H_{*}\left(X ; \mathbb{Z}_{2}\right)$.

Theorem B Let $M$ be either a compact Cartan hypercontact 3-manifold (with Reeb vector fields $v_{i}$ ) or the 3 -torus (with a constant frame $v_{i}$ ). Let $X$ be a compact flat hyperkähler manifold and fix a class $\tau \in \pi_{0}(\mathcal{F})$. Then, for a generic perturbation $H: X \times M \rightarrow \mathbb{R}$, there is a natural Floer homology group $\mathrm{HF}_{*}(M, X, \tau ; H)$ associated to a chain complex generated by the solutions of (5) where the boundary operator is defined by counting the solutions of (6). The Floer homology groups associated to different choices of $H$ are naturally isomorphic. Moreover, for the component $\tau_{0}$ of the constant maps there is a natural isomorphism $\operatorname{HF}_{*}\left(M, X, \tau_{0} ; H\right) \cong H_{*}\left(X ; \mathbb{Z}_{2}\right)$. 
Remark The precise condition we need for extending the standard techniques of Floer theory to our setting is that $X$ has nonpositive sectional curvature. As every hyperkähler manifold has vanishing Ricci tensor, nonpositive sectional curvature implies that $X$ is flat and hence is a quotient of a hyperkähler torus by a finite group. An example is the quotient of the standard 12 -torus $\mathbb{H}^{3} / \mathbb{Z}^{12}$ by the $\mathbb{Z}_{2}$-action determined by $(x, y, z) \mapsto(y, x, z+1 / 2)$.

\section{A more general setting}

There is conjecturally a much richer theory which provides Floer homological invariants for all triples $(M, X, \tau)$, consisting of a Cartan hypercontact 3-manifold $M$, a compact hyperkähler manifold $X$, and a homotopy class $\tau$ of maps from $M$ to $X$. One basic observation is that every holomorphic sphere in a hyperkähler manifold gives rise to a solution of (4) on $M=S^{3}$. Another point is that $\pi_{3}(X)$ can be a very rich group. For example, the third homotopy group of the K3 surface has 253 generators (see Cochran-Habegger [3, Appendix]).

Example Think of the 3 -sphere as the unit sphere in the quaternions $\mathbb{H} \cong \mathbb{R}^{4}$ and of the 2 -sphere as the unit sphere in the imaginary quaternions $\operatorname{Im}(\mathbb{H}) \cong \mathbb{R}^{3}$. For $\lambda=$ $\lambda_{1} \mathbf{i}+\lambda_{2} \mathbf{j}+\lambda_{3} \mathbf{k} \in S^{2}$ denote $J_{\lambda}:=\lambda_{1} I+\lambda_{2} J+\lambda_{3} K$ and $\omega_{\lambda}=\lambda_{1} \omega_{1}+\lambda_{2} \omega_{2}+\lambda_{3} \omega_{3}$. Define $h_{\lambda}: S^{3} \rightarrow S^{2}$ by $h_{\lambda}(y):=-\bar{y} \lambda y$. If $u: S^{2} \rightarrow X$ is a $J_{\lambda}$-holomorphic sphere then

$$
f:=u \circ h_{\lambda}: S^{3} \rightarrow X
$$

is a critical point of $\mathcal{A}$ and

$$
E(u)=\frac{1}{2} \int_{S^{2}}|d u|^{2}=\int_{S^{2}} u^{*} \omega_{\lambda}=\frac{1}{2 \pi} \mathcal{A}\left(u \circ h_{\lambda}\right) .
$$

To see this, assume $\lambda=\mathbf{i}$ and write $h_{1}(y):=-\bar{y} \mathbf{i} y, h_{2}(y):=-\bar{y} \mathbf{j} y$, and $h_{3}(y):=$ $-\bar{y} \mathbf{k} y$. These functions satisfy $\partial_{v_{i}} h_{i}=0$ and $\partial_{v_{j}} h_{i}=-\partial_{v_{i}} h_{j}=2 h_{k}$ for every cyclic permutation $i, j, k$ of $1,2,3$. Hence $h_{1} \wedge \partial_{v_{3}} h_{1}=\partial_{v_{2}} h_{1}$. If $u: S^{2} \rightarrow X$ is an $I-$ holomorphic sphere it follows that the function $f:=u \circ h_{1}$ satisfies $\partial_{v_{1}} f=0$ and $I \partial_{v_{3}} f=\partial_{v_{2}} f$ and hence is a solution of (4). Moreover, $2 \pi \int_{S^{2}} \sigma=-\int_{S^{3}} \alpha_{1} \wedge h_{1}^{*} \sigma$ for $\sigma \in \Omega^{2}\left(S^{2}\right)$. (When $\sigma$ is exact both sides are zero. Since $-\alpha_{1} \wedge h_{1}^{*} \mathrm{dvol}_{S^{2}}=$ $4 \mathrm{dvol}_{S^{3}}$ the value of the factor follows from $\operatorname{Vol}\left(S^{2}\right)=4 \pi$ and $\operatorname{Vol}\left(S^{3}\right)=2 \pi^{2}$.) With $\sigma=u^{*} \omega_{1}$ this implies $2 \pi \int_{S^{2}} u^{*} \omega_{1}=-\int_{S^{2}} \alpha_{1} \wedge h_{1}^{*} u^{*} \omega_{1}=\mathcal{A}\left(u \circ h_{1}\right)$. Here the last equation follows from the fact that $u^{*} \omega_{2}=u^{*} \omega_{3}=0$ for every $I$-holomorphic curve $u$.

The main technical difficulty in setting up the Floer theory for general hyperkähler manifolds is to establish a suitable compactness theorem. In contrast to the familiar 
theory the derivatives for a sequence of solutions of (5) or (6) will not just blow up at isolated points but along codimension-2 subsets. For example, if $u_{v}: S^{2} \rightarrow X$ is a sequence of $I$-holomorphic curves and $h: S^{3} \rightarrow S^{2}$ is a suitable Hopf fibration, then $f_{v}:=u_{v} \circ h$ is a sequence of solutions of (4) and its derivatives blow up along the Hopf circle $h^{-1}\left(z_{0}\right)$ whenever the derivatives of $u_{v}$ blow up near $z_{0}$. This phenomenon is analogous to the codimension 4 bubbling in Donaldson-Thomas theory [7].

\section{Floer-Donaldson theory}

Let $\Sigma$ be a hyperkähler 4-manifold with complex structures $\mathbf{i}, \mathbf{j}, \mathbf{k}$ and symplectic forms $\sigma_{1}, \sigma_{2}, \sigma_{3}$. Consider the elliptic partial differential equation

$$
d u-I d u \mathbf{i}-J d u \mathbf{j}-K d u \mathbf{k}=0
$$

for smooth maps $u: \Sigma \rightarrow X$. This is sometimes called the Cauchy-Riemann-Fueter equation and it has been widely studied (see Taubes [29], Haydys [18, Chapter 3; 19] and references). For $\Sigma=\mathbb{R} \times M$ with its standard hyperkähler structure (see below) Equation (9) is equivalent to (6) with $H=0$. The solutions of (9) satisfy the energy identity

$$
E(u)=\frac{1}{8} \int_{\Sigma}|d u-I d u \mathbf{i}-J d u \mathbf{j}-K d u \mathbf{k}|^{2} \operatorname{dvol}_{\Sigma}-\int_{\Sigma} \sum_{i=1}^{3} \sigma_{i} \wedge u^{*} \omega_{i},
$$

where $E(u):=\frac{1}{2} \int_{\Sigma}|d u|^{2} \mathrm{dvol}_{\Sigma}$. The linearized operator

$$
\mathcal{D}_{u}: \Omega^{0}\left(\Sigma, u^{*} T X\right) \rightarrow \Omega_{\mathbb{H}}^{1}\left(\Sigma, u^{*} T X\right)
$$

takes values in the space of 1 -forms on $\Sigma$ with values in $u^{*} T X$ that are complex linear with respect to $I, J$, and $K$. When $\Sigma$ is closed this operator is Fredholm between appropriate Sobolev completions and its index is

$$
\operatorname{ind}\left(\mathcal{D}_{u}\right)=-\left\langle c_{2}(T X), u_{*}[\Sigma]\right\rangle+\frac{\chi(\Sigma)}{24} \operatorname{dim}^{\mathbb{R}} X,
$$

where $\chi(\Sigma)$ is the Euler characteristic. Equation (11) continues to hold in the case $\Sigma=$ $S^{1} \times M$ with its natural quaternionic structure. We sketch a proof below. Conjecturally, there should be Gromov-Witten type invariants obtained from intersection theory on the moduli space of solutions of (9).

It is also possible to consider hyperkähler 4-manifolds $\Sigma$ with cylindrical ends $\iota^{ \pm}: \mathbb{R}^{ \pm} \times M^{ \pm} \rightarrow \Sigma$. Here we assume that $M^{ \pm}$is either a Cartan hypercontact 3-manifold or a 3-torus. Then $\mathbb{R}^{ \pm} \times M^{ \pm}$has a natural flat hyperkähler structure; see Donaldson [5] and Geiges-Gonzalo [14]. In the hypercontact case the symplectic 
forms are $\omega_{i}=\kappa^{-1} d\left(e^{-\kappa s} \alpha_{i}\right)=e^{-\kappa s}\left(-d s \wedge \alpha_{i}+\alpha_{j} \wedge \alpha_{k}\right)$ and in the torus case they are $\omega_{i}=-d s \wedge \alpha_{i}+\alpha_{j} \wedge \alpha_{k}$ for every cyclic permutation $i, j, k$ of $1,2,3$. In both cases the complex structure $\mathbf{i}$ is given by $\partial_{s} \mapsto-v_{1}, v_{1} \mapsto \partial_{s}, v_{2} \mapsto v_{3}$, $v_{3} \mapsto-v_{2}$ and similarly for $\mathbf{j}$ and $\mathbf{k}$. We assume that the embeddings $\iota^{ \pm}$are hyperkähler isomorphisms onto their images and that the complement $\Sigma \backslash\left(\mathrm{im} \iota^{+} \cup \operatorname{im} \iota^{-}\right)$has a compact closure. Alternatively, it might also be interesting to consider hyperkähler 4-manifolds with asymptotically cylindrical ends as in Kronheimer [20; 21]. One can then (conjecturally) use the solutions of Equation (9) with Hamiltonian perturbations on the cylindrical ends to obtain a homomorphism $\operatorname{HF}_{*}\left(M^{-}, X\right) \rightarrow \operatorname{HF}_{*}\left(M^{+}, X\right)$ respectively $\mathrm{HF}^{*}\left(M^{+}, X\right) \rightarrow \operatorname{HF}^{*}\left(M^{-}, X\right)$.

Proof of the index formula We relate $\mathcal{D}_{u}$ to a Dirac operator on $\Sigma$ associated to a $\operatorname{spin}^{c}$ structure. On $\Sigma$ we have a Hermitian vector bundle $W=W^{+} \oplus W^{-}$where

$$
W^{+}:=u^{*} T X \oplus u^{*} T X, \quad W^{-}:=\operatorname{Hom}_{\mathbb{H}}\left(T \Sigma, u^{*} T X\right) \oplus \operatorname{Hom}_{I}\left(T \Sigma, u^{*} T X\right) .
$$

Here $\operatorname{Hom}_{\mathbb{H}}\left(T \Sigma, u^{*} T X\right)$ denotes the bundle of quaternionic homomorphisms and $\operatorname{Hom}_{I}\left(T \Sigma, u^{*} T X\right)$ denotes the bundle of homomorphisms that are complex linear with respect to $I$ and complex antilinear with respect to $J$ and $K$. The complex structures on $W^{+}$and $W^{-}$are given by $\left(\xi_{1}, \xi_{2}\right) \mapsto\left(I \xi_{2}, I \xi_{1}\right)$. The $\operatorname{spin}^{c}$ structure $\Gamma: T \Sigma \rightarrow \operatorname{End}(W)$ has the form

$$
\Gamma(v):=\left(\begin{array}{cc}
0 & -\gamma(v)^{*} \\
\gamma(v) & 0
\end{array}\right)
$$

for $v \in T_{z} \Sigma$ where $\gamma(v): W_{z}^{+} \rightarrow \mathbb{W}_{z}^{-}$is given by

$$
\gamma(v)\left(\xi_{1}, \xi_{2}\right):=\left(\pi_{\mathbb{H}}\left(\langle v, \cdot\rangle \xi_{1}\right), \pi_{I}\left(\langle v, \cdot\rangle \xi_{2}\right)\right) .
$$

Here $\pi_{\mathbb{H}}, \pi_{I}: \operatorname{Hom}_{\mathbb{R}}\left(T \Sigma, u^{*} T X\right) \rightarrow \operatorname{Hom}_{\mathbb{R}}\left(T \Sigma . u^{*} T X\right)$ denote the projections

$$
\pi_{\mathbb{H}}(A):=A-I A \mathbf{i}-J A \mathbf{j}-K A \mathbf{k}, \quad \pi_{I}(A):=A-I A \mathbf{i}+J A \mathbf{j}+K A \mathbf{k} .
$$

The Dirac operator $D: \Omega^{0}\left(\Sigma, W^{+}\right) \rightarrow \Omega^{0}\left(\Sigma, W^{-}\right)$is the direct sum of $\mathcal{D}_{u}$ and $\widetilde{\mathcal{D}}_{u}: \Omega^{0}\left(\Sigma, u^{*} T X\right) \rightarrow \Omega_{I}^{1}\left(\Sigma, u^{*} T X\right)$ given by $\widetilde{\mathcal{D}}_{u} \xi:=\pi_{I}(\nabla \xi)$. These operators have the same index and hence

$$
\operatorname{ind}^{\mathbb{R}}\left(\mathcal{D}_{u}\right)=\operatorname{ind}^{\mathbb{R}}(D)=\frac{\operatorname{rank}^{\mathbb{R}}\left(W^{+}\right)}{24} \chi(\Sigma)+\frac{1}{2}\left\langle c_{1}\left(W^{+}\right)^{2}-2 c_{2}\left(W^{+}\right),[\Sigma]\right\rangle .
$$

The last equation follows from the Atiyah-Singer index theorem (see [23]). Alternatively, one can identify $\Omega^{0}\left(\Sigma, W^{+}\right)$with $\Omega^{0,0}\left(\Sigma, u^{*} T X\right) \oplus \Omega^{2,0}\left(u^{*} T X\right)$ via $\left(\xi_{1}, \xi_{2}\right) \mapsto\left(\xi_{1}+\xi_{2}, J\left(\xi_{2}-\xi_{1}\right) \omega_{\mathbf{j}}+K\left(\xi_{2}-\xi_{1}\right) \omega_{\mathbf{k}}\right)$ and the space $\Omega^{0}\left(\Sigma, W^{-}\right)$with 
$\Omega^{1,0}\left(\Sigma, u^{*} T X\right)$ via $\left(\alpha_{1}, \alpha_{2}\right) \rightarrow \alpha_{1}+\alpha_{2}$. Under these identifications the Dirac operator $D$ corresponds to the twisted Cauchy-Riemann operator

$$
\partial+\partial^{*}: \Omega^{\mathrm{ev}, 0}\left(\Sigma, u^{*} T X\right) \rightarrow \Omega^{\mathrm{odd}, 0}\left(\Sigma, u^{*} T X\right) .
$$

Since $I$ is homotopic to $-I$, the complex Fredholm index of $D$ is the holomorphic Euler characteristic of the bundle $u^{*} T X \rightarrow \Sigma$ and, by the Hirzebruch-Riemann-Roch formula,

$$
\operatorname{ind}^{\mathbb{R}}\left(\mathcal{D}_{u}\right)=\operatorname{index}^{\mathbb{C}}(D)=\int_{\Sigma} \operatorname{ch}\left(u^{*} T X\right) \operatorname{td}(T \Sigma) .
$$

With $\mathrm{ch}=\operatorname{rank}^{\mathbb{C}}+c_{1}+\frac{1}{2}\left(c_{1}^{2}-2 c_{2}\right)$ and $\mathrm{td}=1+\frac{1}{2} c_{1}+\frac{1}{12}\left(c_{1}^{2}+c_{2}\right)$ this gives again the above formula, and (11) follows because $c_{1}(T X)=c_{1}(T \Sigma)=0$.

\section{Ring structure}

As an example of this construction we obtain (conjecturally) a ring structure on $\operatorname{HF}^{*}\left(S^{3}, X\right)$. Take $\Sigma:=\mathbb{H} \backslash\left\{-\frac{1}{2}, \frac{1}{2}\right\}$ and define $\iota^{-}:(-\infty, 0] \times S^{3} \rightarrow \mathbb{H}$ by

$$
\iota^{-}(s, y):=e^{-s} y \text {. }
$$

The image of this map is the complement of the open unit ball in $\mathbb{H}$. The embed$\operatorname{ding} \iota^{+}:[0, \infty) \times\left(S^{3} \sqcup S^{3}\right) \rightarrow \mathbb{H}$ is the disjoint union of the embeddings $(s, y) \mapsto$ $e^{-1-s} y \pm \frac{1}{2}$. The resulting quaternionic pair of pants product

$$
\operatorname{HF}^{*}\left(S^{3}, X\right) \otimes \operatorname{HF}^{*}\left(S^{3}, X\right) \rightarrow \operatorname{HF}^{*}\left(S^{3}, X\right)
$$

should be independent of the choice of the embeddings and the Hamiltonian perturbations used to define it. Moreover, counting the solutions of (9) on the punctured cylinder $\mathbb{R} \times M \backslash\{\mathrm{pt}\}$, will lead to a module structure of $\operatorname{HF}^{*}(M, X)$ over $\operatorname{HF}^{*}\left(S^{3}, X\right)$ for every $M$.

The compactness and transversality results in the present paper show that this construction is perfectly rigorous and gives rise to an associative product on $\operatorname{HF}^{*}\left(S^{3}, X\right)$ whenever $X$ is flat. Moreover, in this case it agrees with the cup product under our isomorphism $\operatorname{HF}^{*}\left(S^{3}, X\right) \cong H^{*}\left(X ; \mathbb{Z}_{2}\right)$.

\section{Relations with Donaldson-Thomas theory}

In [7] Donaldson and Thomas outline the construction of Donaldson type invariants of 8 dimensional Spin(7)-manifolds $Z$ and Floer homological invariants of 7-dimensional $\mathrm{G}_{2}$-manifolds $Y$. In the case $Z=\Sigma \times S$, where $\Sigma$ and $S$ are hyperkähler surfaces, they explain that solutions of their equation on $\Sigma \times S$ correspond, in the adiabatic limit where the metric on $S$ degenerates to zero, to solutions $u: \Sigma \rightarrow \mathcal{M}(S)$ of (9) with 
values in a suitable moduli space $X=\mathcal{M}(S)$ of bundles over $S$. In a similar vein there is a conjectural correspondence between the Donaldson-Thomas-Floer theory of

$$
Y=M \times S
$$

with the Floer homology groups $\operatorname{HF}_{*}(M, \mathcal{M}(S))$ discussed above whenever $M$ is either a Cartan hypercontact 3-manifold or a flat 3-torus. Namely, the solutions of the Floer equation in Donaldson-Thomas theory on $\mathbb{R} \times Y$ with $Y=M \times S$ correspond, in the adiabatic limit, formally to the solutions of (6) on $\mathbb{R} \times M$ with values in $\mathcal{M}(S)$.

\section{Boundary value problems}

If $M$ is Cartan hypercontact 3-manifold with boundary $\partial M$ and Reeb vector fields $v_{1}, v_{2}, v_{3}$ then there is a unique map $\lambda: \partial M \rightarrow S^{2}$ such that

$$
v:=\sum_{i} \lambda_{i} v_{i}: \partial M \rightarrow T M
$$

is the outward pointing unit normal vector field. In this case the 1 -form (1) is not closed. Its differential is given by the formula

$$
T_{f} \mathcal{F} \times T_{f} \mathcal{F} \rightarrow \mathbb{R}:\left(\widehat{f}_{1}, \widehat{f}_{2}\right) \mapsto \int_{\partial M} \omega_{\lambda}\left(\widehat{f}_{1}, \widehat{f}_{2}\right) \operatorname{dvol}_{\partial M}
$$

This is a symplectic form on the space of maps $\partial M \rightarrow X$. Thus it seems natural to impose the Lagrangian boundary condition

$$
f(y) \in L_{y}, \quad y \in \partial M,
$$

where $\bigsqcup_{y \in \partial M} L_{y}$ is a smooth submanifold of $\partial M \times X$ such that $L_{y}$ is Lagrangian with respect to $\omega_{\lambda(y)}$ for every $y \in \partial M$. In this paper we do not carry out the analysis for this boundary value problem.

In the technical parts of this paper we shall restrict the discussion to the case where $M$ is a (Cartan) hypercontact 3-manifold. The analysis for the case $M=\mathbb{T}^{3}$ is almost verbatim the same and in some places easier because the metric is flat. In Section 2 we introduce the hypersymplectic action functional and its critical points, discuss the Floer equation, and restate Theorem A. In Section 3 we prove the main compactness and exponential decay theorems for the solutions of (5) and (6). These results are only valid for flat target manifolds $X$. The details of the transversality theory are worked out in Section 4 (for general target manifolds $X$ ). With compactness and transversality established, the construction of Floer homology is completely standard and we restrict ourselves to restating the result in Section 5. However, the computation of Floer homology still requires some serious analysis which is carried out in Section 5. 
Three appendices discuss basic properties of hypercontact 3-manifolds, the relevant a priori estimates, and a removable singularity theorem.

Acknowledgements Thanks to Ron Stern for pointing out to us the discussion of $\pi_{3}(X)$ for a simply connected 4-manifold $X$ in Cochran-Habegger [3]. Thanks to Oliver Baues, Kenji Fukaya, Hansjoerg Geiges and Katrin Wehrheim for helpful comments. Sonja Hohloch and Gregor Noetzel would like to thank the Forschungsinstitut für Mathematik at ETH Zürich for its hospitality. We thank the referee for helpful suggestions.

\section{The hypersymplectic action functional}

Let $X$ be a hyperkähler manifold with complex structures $I, J, K$ and associated symplectic forms $\omega_{1}, \omega_{2}, \omega_{3}$. Let $\left(M, \alpha_{1}, \alpha_{2}, \alpha_{3}\right)$ be a positive hypercontact 3-manifold with Reeb vector fields $v_{1}, v_{2}, v_{3}$ (see Appendix A). Then the space $\mathcal{F}:=\operatorname{Map}(M, X)$ of smooth maps $f: M \rightarrow X$ carries a natural hypersymplectic action functional $\mathcal{A}: \mathcal{F} \rightarrow \mathbb{R}$ given by

$$
\mathcal{A}(f):=-\int_{M}\left(\alpha_{1} \wedge f^{*} \omega_{1}+\alpha_{2} \wedge f^{*} \omega_{2}+\alpha_{3} \wedge f^{*} \omega_{3}\right)
$$

The next lemma shows that the critical points of $\mathcal{A}$ are the solutions of the partial differential equation

$$
\not(f):=I d f\left(v_{1}\right)+J d f\left(v_{2}\right)+K d f\left(v_{3}\right)=0 .
$$

This is a Dirac type elliptic equation because the vector fields $v_{i}$ are everywhere linearly independent (see Lemma A.1) and the complex structures $I, J, K$ satisfy the quaternionic relations. (The square of $\not$ in local coordinates is a standard second order elliptic operator.)

Lemma 2.1 The differential of $\mathcal{A}$ along a path $\mathbb{R} \rightarrow \mathcal{F}: t \mapsto f_{t}$ is

$$
\frac{d}{d t} \mathcal{A}\left(f_{t}\right)=\int_{M}\left\langle\partial_{t} f_{t}, \not \partial\left(f_{t}\right)\right\rangle \kappa \operatorname{dvol}_{M},
$$

where $\kappa$ and the metric on $M$ are as in Remark A.2.

Proof By Cartan's formula, we have

$$
\frac{d}{d t} f_{t}^{*} \omega_{i}=d \beta_{i}, \quad \beta_{i}:=\omega_{i}\left(\partial_{t} f, d f_{t} \cdot\right),
$$


for $i=1,2,3$. Hence

$$
\begin{aligned}
\frac{d}{d t} \mathcal{A}\left(f_{t}\right) & =-\int_{M} \sum_{i} \alpha_{i} \wedge d \beta_{i}=-\int_{M} \sum_{i} d \alpha_{i} \wedge \beta_{i} \\
& =-\int_{M}\left(\sum_{i} d \alpha_{i} \wedge \beta_{i}\right)\left(v_{1}, v_{2}, v_{3}\right) \mathrm{dvol}_{M} \\
& =-\int_{M} \kappa \sum_{i} \beta_{i}\left(v_{i}\right) \operatorname{dvol}_{M} \\
& =\int_{M} \kappa\left\langle\partial_{t} f_{t}, I d f_{t}\left(v_{1}\right)+J d f_{t}\left(v_{2}\right)+K d f_{t}\left(v_{3}\right)\right\rangle \operatorname{dvol}_{M} .
\end{aligned}
$$

Here the second equation follows from integration by parts, the third equation follows from the fact that $v_{1}, v_{2}, v_{3}$ form an orthonormal basis, the fourth follows from the definition of $\kappa$ in Remark A.2, and the last equation uses the definition of $\beta_{i}$ and the hyperkähler structure of $X$. This proves the lemma.

\section{The energy identity}

The energy of a smooth function $f: M \rightarrow X$ is defined by

$$
\mathcal{E}(f):=\frac{1}{2} \int_{M}|d f|^{2} \operatorname{dvol}_{M}=\frac{1}{2} \int_{M} \sum_{i=1}^{3}\left|d f\left(v_{i}\right)\right|^{2} \operatorname{dvol}_{M} .
$$

Lemma 2.2 The energy of a smooth function $f: M \rightarrow X$ is related to the hypersymplectic action via

$$
\mathcal{E}(f)=\mathcal{A}(f)+\frac{1}{2} \int_{M}|\partial(f)|^{2} \operatorname{dvol}_{M}-\int_{M}\left\langle\partial(f), d f\left(v_{0}\right)\right\rangle \mathrm{dvol}_{M},
$$

where

$$
v_{0}:=\alpha_{2}\left(v_{3}\right) v_{1}+\alpha_{3}\left(v_{1}\right) v_{2}+\alpha_{1}\left(v_{2}\right) v_{3} .
$$

In particular $\mathcal{E}(f)=\mathcal{A}(f)$ for every solution of (13).

Remark 2.3 The vector field $v_{0}$ vanishes if and only if $\alpha_{i}\left(v_{j}\right)=\delta_{i j}$. If this holds then, for every $f \in \mathcal{F}$, we have

$$
\mathcal{E}(f)=\mathcal{A}(f)+\frac{1}{2} \int_{M}|\not(f)|^{2} \mathrm{dvol}_{M} .
$$

Hence the energy of $f$ is controlled by the $L^{2}$ norm of $\not(f)=\operatorname{grad} \mathcal{A}(f)$ and the action. 
Proof of Lemma 2.2 By direct calculation (dropping the term dvol $_{M}$ ) we obtain

$$
\begin{aligned}
\frac{1}{2} \int_{M}|\partial(f)|^{2} & -\mathcal{E}(f) \\
& =\frac{1}{2} \int_{M}\left(\left|I d f\left(v_{1}\right)+J d f\left(v_{2}\right)+K d f\left(v_{3}\right)\right|^{2}-\sum_{i}\left|d f\left(v_{i}\right)\right|^{2}\right) \\
& =\int_{M}\left(\left\langle K d f\left(v_{1}\right), d f\left(v_{2}\right)\right\rangle+\left\langle I d f\left(v_{2}\right), d f\left(v_{3}\right)\right\rangle+\left\langle J d f\left(v_{3}\right), d f\left(v_{1}\right)\right\rangle\right) \\
& =\int_{M}\left(f^{*} \omega_{1}\left(v_{2}, v_{3}\right)+f^{*} \omega_{2}\left(v_{3}, v_{1}\right)+f^{*} \omega_{3}\left(v_{1}, v_{2}\right)\right) .
\end{aligned}
$$

On the other hand

$$
\begin{aligned}
& \int_{M}\left\langle\not \partial(f), d f\left(v_{0}\right)\right\rangle-\mathcal{A}(f) \\
&=\int_{M}\left\langle\partial(f), \alpha_{2}\left(v_{3}\right) d f\left(v_{1}\right)+\alpha_{3}\left(v_{1}\right) d f\left(v_{2}\right)+\alpha_{1}\left(v_{2}\right) d f\left(v_{3}\right)\right\rangle-\mathcal{A}(f) \\
&=\int_{M} \alpha_{2}\left(v_{3}\right)\left(f^{*} \omega_{2}\left(v_{2}, v_{1}\right)+f^{*} \omega_{3}\left(v_{3}, v_{1}\right)\right) \\
&+\int_{M} \alpha_{3}\left(v_{1}\right)\left(f^{*} \omega_{1}\left(v_{1}, v_{2}\right)+f^{*} \omega_{3}\left(v_{3}, v_{2}\right)\right) \\
&+\int_{M} \alpha_{1}\left(v_{2}\right)\left(f^{*} \omega_{1}\left(v_{1}, v_{3}\right)+f^{*} \omega_{2}\left(v_{2}, v_{3}\right)\right) \\
&+\int_{M}\left(\alpha_{1} \wedge f^{*} \omega_{1}+\alpha_{2} \wedge f^{*} \omega_{2}+\alpha_{3} \wedge f^{*} \omega_{3}\right) \\
&= \int_{M}\left(f^{*} \omega_{1}\left(v_{2}, v_{3}\right)+f^{*} \omega_{2}\left(v_{3}, v_{1}\right)+f^{*} \omega_{3}\left(v_{1}, v_{2}\right)\right) .
\end{aligned}
$$

The last equation follows by inserting the vector fields $v_{1}, v_{2}, v_{3}$ into the 3 -forms $\alpha_{i} \wedge f^{*} \omega_{i}$. This proves the lemma.

\section{The Hessian}

The tangent space of $\mathcal{F}$ at $f$ is the space of vector fields along $f$ :

$$
T_{f} \mathcal{F}=\operatorname{Vect}(f)=\Omega^{0}\left(M, f^{*} T X\right) .
$$

It is convenient to use the inner product

$$
\langle\xi, \eta\rangle_{L^{2}}:=\int_{M}\langle\xi, \eta\rangle \kappa \operatorname{dvol}_{M}
$$


on this space. One reason for this choice is the formula in Lemma 2.1. Another is the following observation.

Lemma 2.4 For every smooth function $f: M \rightarrow \mathbb{R}$ we have

$$
\int_{M} d f\left(v_{i}\right) \kappa \mathrm{dvol}_{M}=0
$$

Thus the covariant divergence of the vector field $v_{i}$ is given by

$$
\operatorname{div}\left(v_{i}\right)=-\kappa^{-1} d \kappa\left(v_{i}\right)
$$

and the operator $\nabla_{v_{i}}: \Omega^{0}(M, E) \rightarrow \Omega^{0}(M, E)$ is skew adjoint with respect to the $L^{2}$ inner product (17) (for every Riemannian vector bundle $E \rightarrow M$ with any Riemannian connection).

Proof The covariant divergence of a vector field $v \in \operatorname{Vect}(M)$ is the function $\operatorname{div}(v): M \rightarrow \mathbb{R}$ defined by $\operatorname{div}(v):=\sum_{j}\left\langle\nabla_{e_{j}} v, e_{j}\right\rangle$ for any orthonormal frame $e_{j}$ of $T M$. It is characterized by the property

$$
\int_{M} d f(v) \operatorname{dvol}_{M}+\int_{M} f \operatorname{div}(v) \operatorname{dvol}_{M}=0
$$

for every function $f: M \rightarrow \mathbb{R}$. Now, for every 1 -form $\beta \in \Omega^{1}(M)$, we have $\left(\beta \wedge d \alpha_{i}\right)\left(v_{1}, v_{2}, v_{3}\right)=\beta\left(v_{i}\right) \kappa$ and hence $\beta \wedge d \alpha_{i}=\beta\left(v_{i}\right) \kappa \mathrm{dvol}_{M}$. With $\beta=d f$ this gives (18). The formula for the covariant divergence of $v_{i}$ follows by replacing $f$ with $\kappa^{-1} f$. This proves the lemma.

Lemma 2.5 The covariant Hessian of $\mathcal{A}$ at $f \in \mathcal{F}$ is the operator

$$
\not D=D_{f}: \Omega^{0}\left(M, f^{*} T X\right) \rightarrow \Omega^{0}\left(M, f^{*} T X\right)
$$

given by

$$
\not{D} \xi:=I \nabla_{v_{1}} \xi+J \nabla_{v_{2}} \xi+K \nabla_{v_{3}} \xi
$$

for $\xi \in \Omega^{0}\left(M, f^{*} T X\right)$. Here $\nabla$ is the Levi-Civita connection of the hyperkähler metric on $X$. The operator $\mathbb{D}: W^{1,2}\left(M, f^{*} T X\right) \rightarrow L^{2}\left(M, f^{*} T X\right)$ is self-adjoint with respect to the $L^{2}$ inner product (17).

Proof The covariant Hessian of $\mathcal{A}$ at $f \in \mathcal{F}$ is defined by the formula $\left.\xi \mapsto \nabla_{t}\left(\not \partial f_{t}\right)\right|_{t=0}$ where $t \mapsto f_{t}$ is a smooth curve in $\mathcal{F}$ with $f_{0}=f$ and $\left.\partial_{t} f_{t}\right|_{t=0}=\xi$. Hence (19) follows from the fact that the complex structures $I, J, K$ are covariant constant and $\nabla$ 
is torsion free. That $\mathbb{D}$ is symmetric with respect to the $L^{2}$ inner product (17) follows from Lemma 2.4. To prove that $\mathbb{D}$ is self-adjoint we observe that its square is given by

$$
\begin{aligned}
\mathcal{D D D} \xi= & -\nabla_{v_{1}} \nabla_{v_{1}} \xi-\nabla_{v_{2}} \nabla_{v_{2}} \xi-\nabla_{v_{3}} \nabla_{v_{3}} \xi \\
& +I\left(R\left(d f\left(v_{2}\right), d f\left(v_{3}\right)\right) \xi-\nabla_{\left[v_{2}, v_{3}\right]} \xi\right) \\
& +J\left(R\left(d f\left(v_{3}\right), d f\left(v_{1}\right)\right) \xi-\nabla_{\left[v_{3}, v_{1}\right]} \xi\right) \\
& +K\left(R\left(d f\left(v_{1}\right), d f\left(v_{2}\right)\right) \xi-\nabla_{\left[v_{1}, v_{2}\right]} \xi\right) .
\end{aligned}
$$

Here $R$ denotes the Riemann curvature tensor on $X$. Since $v_{1}, v_{2}, v_{3}$ are linearly independent $\mathbb{D}^{2}$ is a standard second order elliptic operator in local coordinates (with leading term in diagonal form) and hence has the usual elliptic regularity properties. In particular, if $\xi \in L^{2}$ and $\mathbb{D} \xi \in L^{2}$, then $\mathbb{D}^{2} \xi \in W^{-1,2}$ and elliptic regularity gives $\xi \in W^{1,2}$. This implies that $\mathbb{D}$ is self-adjoint as an operator on $L^{2}$ with domain $W^{1,2}$, as claimed.

As in symplectic and instanton Floer theories it is a fundamental observation that the action functional is unbounded above and below and that the operator $\mathcal{D}$ has infinitely positive and negative eigenvalues.

Remark 2.6 If the symplectic forms $\omega_{i}=d \lambda_{i}$ on $X$ are exact then the hypersymplectic action functional can be written in the form

$$
\mathcal{A}(f)=\int_{M} \sum_{i=1}^{3} \lambda_{i}\left(\partial_{v_{i}} f\right) \kappa \mathrm{dvol}_{M} .
$$

The archetypal example is the space $X=\mathbb{H}$ of quaternions with the standard hyperkähler structure. In this case the operator $f \mapsto \partial(f)=\mathscr{D} f$ is linear and the hypersymplectic action is the associated quadratic form

$$
\mathcal{A}(f)=\frac{1}{2} \int_{M}\langle f, \not D f\rangle \kappa \mathrm{dvol}_{M}
$$

Since $\mathcal{A}(f)=0$ for every real valued function $f: M \rightarrow \mathbb{R} \subset \mathbb{H}$ it follows that the negative and positive eigenspaces of $\mathcal{D}$ are both infinite dimensional. In the case $M=S^{3}$ with the standard hypercontact structure, specific eigenfunctions are $f(y)=y$ with eigenvalue $-3, f(y)=y+2 \bar{y}$ with eigenvalue 1 , and $f(y)=\iota \circ h(y)$ where $h: S^{3} \rightarrow S^{2}$ is a suitable Hopf fibration and $\iota: S^{2} \rightarrow \mathbb{H}$ is the inclusion of the 2 -sphere into the imaginary quaternions; in the last example the eigenvalue is -4 . 


\section{Perturbations}

Let $H: X \times M \rightarrow \mathbb{R}$ be a smooth function and define the perturbed hypersymplectic action functional $\mathcal{A}_{H}: \mathcal{F} \rightarrow \mathbb{R}$ by

$$
\mathcal{A}_{H}(f):=-\int_{M} \sum_{i=1}^{3} \alpha_{i} \wedge f^{*} \omega_{i}-\int_{M} H(f) \kappa \mathrm{dvol}_{M} .
$$

Here we write $H(f)$ for the function $M \rightarrow \mathbb{R}: y \mapsto H(f(y), y)$. For $y \in M$ let $H_{y}:=H(\cdot, y)$ and denote by $\nabla H(\cdot, y):=\nabla H_{y}$ the gradient of $H$ with respect to the first argument. Then, by Lemma 2.1, the critical points of $\mathcal{A}_{H}$ are the solutions of the perturbed equation

$$
I d f\left(v_{1}\right)+J d f\left(v_{2}\right)+K d f\left(v_{3}\right)=\nabla H(f) .
$$

Here we denote by $\nabla H(f)$ the vector field $y \mapsto \nabla H_{y}(f(y))$ along $f$. By Lemma 2.2 , every solution of (21) satisfies the inequality

$$
\mathcal{A}_{H}(f) \geq-\int_{M}\left(\kappa H(f)+\frac{1}{2}|\nabla H(f)|^{2}\right) \mathrm{dvol}_{M} .
$$

\section{Gradient flow lines}

By Lemma 2.1, the gradient of $\mathcal{A}_{H}$ with respect to the $L^{2}$ inner product (17) is given by

$$
\operatorname{grad} \mathcal{A}_{H}(f)=\operatorname{Idf}\left(v_{1}\right)+J d f\left(v_{2}\right)+K d f\left(v_{3}\right)-\nabla H(f)=: \partial_{H}(f) .
$$

Hence the negative gradient flow lines of $\mathcal{A}_{H}$ are the solutions $u$ : $\mathbb{R} \times M \rightarrow X$ of the partial differential equation

$$
\partial_{s} u+I \partial_{v_{1}} u+J \partial_{v_{2}} u+K \partial_{v_{3}} u=\nabla H(u) .
$$

The energy of a smooth map $u: \mathbb{R} \times M \rightarrow X$ is defined by

$$
\mathcal{E}_{H}(u):=\frac{1}{2} \int_{\mathbb{R} \times M}\left(\left|\partial_{s} u\right|^{2}+\left|\partial_{H}(u)\right|^{2}\right) \kappa \operatorname{dvol}_{M} d s .
$$

As in finite dimensional Morse theory and Floer homology, the finite energy solutions of (22) are the ones that converge to critical points of the perturbed hypersymplectic action functional as $s$ tends to $\pm \infty$ (see Theorem 3.13 below). Thus, in the case $\mathcal{E}_{H}(u)<\infty$, there are solutions $f^{ \pm}: M \rightarrow X$ of Equation (21) such that $\lim _{s \rightarrow \pm \infty} \partial_{s} u(s, y)=0$, uniformly in $y$, and

$$
\lim _{s \rightarrow \pm \infty} u(s, y)=f^{ \pm}(y), \quad \lim _{s \rightarrow \pm \infty} \mathcal{A}_{H}(u(s, \cdot))=\mathcal{A}_{H}\left(f^{ \pm}\right) .
$$


Moreover the solutions of (22) minimize the energy $\mathcal{E}_{H}(u)$ subject to (23) and their energy is $\mathcal{E}_{H}(u)=\mathcal{A}_{H}\left(f^{-}\right)-\mathcal{A}_{H}\left(f^{+}\right)$.

\section{Moduli spaces}

A solution $f$ of $\partial_{H}(f)=0$ is called nondegenerate if the perturbed Hessian

$$
\mathbb{D}_{f, H} \xi:=I \nabla_{v_{1}} \xi+J \nabla_{v_{2}} \xi+K \nabla_{v_{3}} \xi-\nabla_{\xi} \nabla H_{y}(f)
$$

is bijective. We shall prove that nondegeneracy can be achieved by a generic choice of the Hamiltonian $H: X \times M \rightarrow \mathbb{R}$ (see Theorem 4.1 below). Assuming this we fix two critical points $f^{ \pm}$of the perturbed hypersymplectic action functional $\mathcal{A}_{H}$ and denote the space of Floer trajectories by

$$
\mathcal{M}\left(f^{-}, f^{+} ; H\right):=\left\{u: \mathbb{R} \times M \rightarrow X \mid u \text { satisfies (22), (23), } \sup _{\mathbb{R} \times M}|d u|<\infty\right\} .
$$

We shall prove, again for a generic choice of the perturbation, that these spaces are smooth finite dimensional manifolds. The proof will involve the linearized operator

$$
\mathcal{D}_{u, H} \xi:=\nabla_{s} \xi+I \nabla_{v_{1}} \xi+J \nabla_{v_{2}} \xi+K \nabla_{v_{3}} \xi-\nabla_{\xi} \nabla H(u) .
$$

As in all other versions of Floer homology the Fredholm index of this operator is the spectral flow of the Hessians along $u$. We shall prove that, when $M$ is a Cartan hypercontact 3-manifold and $X$ is flat all the known analysis of symplectic Floer theory carries over to the present setting and gives rise to Floer homology groups that are isomorphic to the singular homology of $X$. This leads to the following existence theorem for solutions of $\partial_{H}(f)=0$. We emphasize that the algebraic count of the solutions gives zero and thus does not provide an existence result.

Theorem 2.7 Let $M$ be a compact Cartan hypercontact 3-manifold and $X$ be a compact flat hyperkähler manifold. If every solution $f$ of $\partial_{H}(f)=0$ is nondegenerate then their number is bounded below by the sum of the Betti numbers of $X$ (with coefficient ring $\mathbb{Z}_{2}$ ). In particular, $\partial_{H}(f)=0$ has a solution for every smooth function $H: X \times M \rightarrow \mathbb{R}$.

Proof See Section 5.

\section{Regularity and compactness}

We assume throughout that $X$ is a compact hyperkähler manifold and $M$ is a compact 3 -manifold equipped with a positive hypercontact structure $\alpha$. Then the Reeb vector 
fields $v_{1}, v_{2}, v_{3}$ form a (positive) orthonormal frame of $T M$ and hence determine a second order elliptic operator

$$
L:=\sum_{i=1}^{3} \mathcal{L}_{v_{i}} \mathcal{L}_{v_{i}}=-d^{*} d-\sum_{i=1}^{3} \operatorname{div}\left(v_{i}\right) \mathcal{L}_{v_{i}}
$$

If $\alpha$ is a Cartan structure then $\operatorname{div}\left(v_{i}\right)=d^{*} \alpha_{i}=0$ (by Lemma 2.4) and so $L$ is the Laplace-Beltrami operator on $M$. In local coordinates $y^{1}, y^{2}, y^{3}$ on $M$ the operator $L$ has the form

$$
L=\sum_{\mu, \nu=1}^{3} a^{\mu \nu} \frac{\partial^{2}}{\partial y^{\mu} \partial y^{\nu}}+\sum_{\nu=1}^{3} b^{\nu} \frac{\partial}{\partial y^{\nu}}
$$

where

$$
a^{\mu \nu}:=\sum_{i=1}^{3} v_{i}^{\mu} v_{i}^{v}, \quad b^{v}:=\sum_{i, \mu=1}^{3} \frac{\partial v_{i}^{v}}{\partial y^{\mu}} v_{i}^{\mu} .
$$

Since the vector fields $v_{i}$ form an orthonormal frame of $T M$, the coefficients $a^{\mu \nu}$ define the Riemannian metric on the cotangent bundle in our local coordinates. The operators $L$ on $M$ and

$$
\mathcal{L}:=\partial_{s} \partial_{s}+L
$$

on $\mathbb{R} \times M$ will play a central role in our study of the solutions of Equations (21) and (22).

Theorem 3.1 (Regularity) If $p>3$ then every $W^{1, p}$ solution $f$ of $\partial_{H}(f)=0$ is smooth. If $p>4$ then every $W^{1, p}$ solution $u$ of (22) is smooth.

Proof For every vector field $v \in \operatorname{Vect}(M)$ we write $\partial_{v} f=\mathcal{L}_{v} f=d f(v)$. Then, for every smooth map $f: M \rightarrow X$ and any two vector fields $v, w$ on $M$, we have $\nabla_{v} \partial_{w} f-\nabla_{w} \partial_{v} f=-\partial_{[v, w]} f$. Hence

$$
\not{D} \not(f)=-\sum_{i=1}^{3} \nabla_{v_{i}} \partial_{v_{i}} f-I \partial_{\left[v_{2}, v_{3}\right]} f-J \partial_{\left[v_{3}, v_{1}\right]} f-K \partial_{\left[v_{1}, v_{2}\right]} f .
$$

In local coordinates $\left(x^{1}, \ldots, x^{m}\right)$ on $X$ and $\left(y^{1}, y^{2}, y^{3}\right)$ on $M$ we have

$$
\left(\nabla_{v} \partial_{w} f\right)^{k}=\sum_{\nu, \mu=1}^{3}\left(\frac{\partial^{2} f^{k}}{\partial y^{\nu} \partial y^{\mu}} v^{\mu} w^{\nu}+\frac{\partial f^{k}}{\partial y^{\nu}} \frac{\partial w^{\nu}}{\partial y^{\mu}} v^{\mu}+\sum_{i, j=1}^{m} \Gamma_{i j}^{k} \frac{\partial f^{i}}{\partial y^{\mu}} \frac{\partial f^{j}}{\partial y^{\nu}} v^{\mu} w^{\nu}\right) .
$$


With $L$ as in (27) this gives

$$
(\mathbb{D} \partial(f))^{k}=-L f^{k}-\sum_{\mu, \nu=1}^{3} \sum_{i, j=1}^{m} \Gamma_{i j}^{k} a^{\mu \nu} \frac{\partial f^{i}}{\partial y^{\mu}} \frac{\partial f^{j}}{\partial y^{\nu}}-g_{f}^{k},
$$

where

$$
g_{f}^{k}:=\sum_{\ell=1}^{m} \sum_{\nu=1}^{3}\left(I_{\ell}^{k}\left[v_{2}, v_{3}\right]^{\nu}+J_{\ell}^{k}\left[v_{3}, v_{1}\right]^{\nu}+K_{\ell}^{k}\left[v_{1}, v_{2}\right]^{\nu}\right) \frac{\partial f^{\ell}}{\partial y^{v}} .
$$

Moreover, the function $h_{f}^{k}:=(\mathcal{D} \partial(f))^{k}=(\mathcal{D} \nabla H(f))^{k}$ is given by

$$
h_{f}^{k}=\sum_{j, \ell=1}^{m} \sum_{\nu=1}^{3} c_{\ell}^{k v}\left(\frac{\partial(\nabla H)^{\ell}}{\partial x^{j}} \frac{\partial f^{j}}{\partial y^{\nu}}+\frac{\partial(\nabla H)^{\ell}}{\partial y^{\nu}}+\sum_{i=1}^{m} \Gamma_{i j}^{\ell}(\nabla H)^{i} \frac{\partial f^{j}}{\partial y^{\nu}}\right),
$$

where

$$
c_{\ell}^{k v}:=I_{\ell}^{k} v_{1}^{v}+J_{\ell}^{k} v_{2}^{v}+K_{\ell}^{k} v_{3}^{v} .
$$

Hence every solution of (21) (of class $W^{1, p}$ ) satisfies the elliptic pde

$$
L f^{k}=-\sum_{\mu, \nu=1}^{3} \sum_{i, j=1}^{m} \Gamma_{i j}^{k} a^{\mu \nu} \frac{\partial f^{i}}{\partial y^{\mu}} \frac{\partial f^{j}}{\partial y^{\nu}}-g_{f}^{k}-h_{f}^{k}
$$

If $f \in W^{1, p}$ for some $p>3$ then the right hand side is in $L^{p / 2}$ and hence, by elliptic regularity, $f$ is of class $W^{2, p / 2}$. By the Sobolev embedding theorem, we then obtain $f \in W^{1, q}$ where $q:=3 p /(6-p)>p$. Continuing by induction, we obtain eventually that $f \in W^{1, q}$ for some $q>6$, hence $f \in W^{2, p}$ and, again by induction, $f \in W^{k, p}$ for every integer $k$.

To prove regularity for the solutions of (22) we introduce the operators

$$
\mathcal{D}:=\nabla_{s}+I \nabla_{v_{1}}+J \nabla_{v_{2}}+K \nabla_{v_{3}}, \quad \mathcal{D}^{*}:=-\nabla_{s}+I \nabla_{v_{1}}+J \nabla_{v_{2}}+K \nabla_{v_{3}} .
$$

Then we have

$$
\begin{aligned}
\mathcal{D}^{*}\left(\partial_{s} u+\partial(u)\right)=-\nabla_{s} \partial_{s} u & -\sum_{i=1}^{3} \nabla_{v_{i}} \partial_{v_{i}} u \\
& -I \partial_{\left[v_{2}, v_{3}\right]} u-J \partial_{\left[v_{3}, v_{1}\right]} u-K \partial_{\left[v_{1}, v_{2}\right]} u .
\end{aligned}
$$

Here we have used (29) and the fact that $\nabla_{s} \partial_{v_{i}} u=\nabla_{v_{i}} \partial_{s} u$ for $i=1,2,3$. If $u$ is a solution of (22) then $\partial_{s} u+\not(u)=\nabla H(u)$. Hence in this case we obtain the equation

$$
\mathcal{L} u^{k}=-\sum_{\mu, \nu=1}^{3} \sum_{i, j=1}^{m} \Gamma_{i j}^{k}(u)\left(\frac{\partial u^{i}}{\partial s} \frac{\partial u^{j}}{\partial s}+a^{\mu \nu} \frac{\partial u^{i}}{\partial y^{\mu}} \frac{\partial u^{j}}{\partial y^{v}}\right)-g_{u}^{k}-h_{u}^{k},
$$


where

$$
\begin{aligned}
\mathcal{L}:= & \frac{\partial^{2}}{\partial s^{2}}+\sum_{\mu, \nu=1}^{3} a^{\mu \nu} \frac{\partial^{2}}{\partial y^{\mu} \partial y^{\nu}}+\sum_{\nu=1}^{3} b^{\nu} \frac{\partial}{\partial y^{\nu}}, \\
g_{u}^{k}:= & \sum_{\ell=1}^{m} \sum_{\nu=1}^{3}\left(I_{\ell}^{k}\left[v_{2}, v_{3}\right]^{\nu}+J_{\ell}^{k}\left[v_{3}, v_{1}\right]^{\nu}+K_{\ell}^{k}\left[v_{1}, v_{2}\right]^{\nu}\right) \frac{\partial u^{\ell}}{\partial y^{\nu}}, \\
h_{u}^{k}:= & \sum_{j, \ell=1}^{m} \sum_{\nu=1}^{3} c_{\ell}^{k v}\left(\frac{\partial(\nabla H)^{\ell}}{\partial x^{j}} \frac{\partial u^{j}}{\partial y^{\nu}}+\frac{\partial(\nabla H)^{\ell}}{\partial y^{\nu}}+\sum_{i=1}^{m} \Gamma_{i j}^{\ell}(\nabla H)^{i} \frac{\partial u^{j}}{\partial y^{\nu}}\right) \\
& -\sum_{j=1}^{m} \frac{\partial(\nabla H)^{k}}{\partial x^{j}} \frac{\partial u^{j}}{\partial s}-\sum_{i, j=1}^{m} \Gamma_{i j}^{k}(u)(\nabla H)^{i} \frac{\partial u^{j}}{\partial s} .
\end{aligned}
$$

If $u \in W^{1, p}$ with $p>4$ then the right hand side in (31) is in $L^{p / 2}$ and so $u \in W^{2, p / 2}$. Thus the Sobolev embedding theorem gives $u \in W^{1, q}$ with $q:=4 p /(8-p)>p$. Continuing by induction we obtain that $u$ is smooth. This proves the theorem.

The bootstrapping argument in the proof of Theorem 3.1 gives rise to uniform estimates for sequences that are bounded in $W^{1, p}$. Hence the Arzéla-Ascoli theorem gives the following compactness result.

\section{Theorem 3.2 Assume $X$ is compact.}

(i) Let $p>3$ and $\Omega \subset M$ be an open set. Then every sequence of solutions $f^{v}: \Omega \rightarrow X$ of Equation (21) that satisfies $\sup _{v}\left\|d f^{v}\right\|_{L^{p}(C)}<\infty$ for every compact set $C \subset \Omega$ has a subsequence that converges in the $C^{\infty}$ topology on every compact subset of $\Omega$.

(ii) Let $p>4$ and $\Omega \subset \mathbb{R} \times M$ be an open set. Then every sequence of solutions $u^{v}: \Omega \rightarrow X$ of Equation (22) that satisfies $\sup _{v}\left\|d u^{v}\right\|_{L^{p}(C)}<\infty$ for every compact set $C \subset \Omega$ has a subsequence that converges in the $C^{\infty}$ topology on every compact subset of $\Omega$.

\section{A priori estimates}

To remove the bounded derivative assumption in Theorem 3.2, at least in the case where $X$ is flat, we establish mean value inequalities for the energy density of the solutions of (22). The solutions of (21) then correspond to the special case $\partial_{s} u \equiv 0$. The mean value inequalities will be based on Theorem B.1 in Appendix B.

Throughout we denote by $L$ and $\mathcal{L}$ the operators (26) and (28) on $M$ and $\mathbb{R} \times M$, respectively, and by $R$ the Riemann curvature tensor on $X$. For a map $u$ : $\mathbb{R} \times M \rightarrow X$ 
we define the energy density $e_{u}: \mathbb{R} \times M \rightarrow \mathbb{R}$ by

$$
e_{u}:=\frac{1}{2}\left|\partial_{s} u\right|^{2}+\frac{1}{2} \sum_{i=1}^{3}\left|\partial_{v_{i}} u\right|^{2}
$$

and we denote by $r_{u}: \mathbb{R} \times M \rightarrow \mathbb{R}$ the sum of the sectional curvatures of suitable coordinate planes tangent to $u$ :

$$
r_{u}:=2 \sum_{j=1}^{3}\left\langle R\left(\partial_{s} u, \partial_{v_{j}} u\right) \partial_{v_{j}} u, \partial_{s} u\right\rangle+\sum_{i, j=1}^{3}\left\langle R\left(\partial_{v_{i}} u, \partial_{v_{j}} u\right) \partial_{v_{j}} u, \partial_{v_{i}} u\right\rangle .
$$

Throughout we fix a Hamiltonian perturbation $H: X \times M \rightarrow \mathbb{R}$. We explicitly do not assume that the hypercontact structure on $M$ is a Cartan structure (unless otherwise mentioned).

Lemma 3.3 There are positive constants $A$ and $B$, depending only on the vector fields $v_{i}$, the metric on $X$, and the Hamiltonian perturbation $H$, such that every solution $u: \mathbb{R} \times M \rightarrow X$ of (22) satisfies the estimate

$$
\mathcal{L} e_{u}+r_{u} \geq-A-B\left(e_{u}\right)^{3 / 2} .
$$

If $H=0$ we obtain an estimate of the form $\mathcal{L} e_{u}+r_{u} \geq-C e_{u}$.

Proof It is convenient to denote the vector field $\partial_{s}$ on $\mathbb{R} \times M$ by $v_{0}$. Then the Lie brackets $\left[v_{0}, v_{j}\right]$ vanish for all $j$, but we shall not use this fact. Abbreviate

$$
w_{1}:=\left[v_{2}, v_{3}\right], \quad w_{2}:=\left[v_{3}, v_{1}\right], \quad w_{3}:=\left[v_{1}, v_{2}\right]
$$

and define the operators

$$
\mathcal{L}^{X}:=\sum_{i=0}^{3} \nabla_{v_{i}} \partial_{v_{i}}, \quad \mathcal{L}^{\nabla}:=\sum_{i=0}^{3} \nabla_{v_{i}} \nabla_{v_{i}} .
$$

Thus $\mathcal{L}^{X}$ acts on maps $u$ : $\mathbb{R} \times M \rightarrow X$ and $\mathcal{L}^{\nabla}$ acts on vector fields along such maps. With this notation every solution $u$ of (22) satisfies the equation

$$
\mathcal{L}^{X} u=-\mathcal{D}^{*} \nabla H(u)-I \partial_{w_{1}} u-J \partial_{w_{2}} u-K \partial_{w_{3}} u,
$$

where $\mathcal{D}^{*}=-\nabla_{s}+I \nabla_{v_{1}}+J \nabla_{v_{2}}+K \nabla_{v_{3}}$. Moreover,

$$
\mathcal{L} e_{u}=\sum_{j=0}^{3}\left\langle\mathcal{L}^{\nabla} \partial_{v_{j}} u, \partial_{v_{j}} u\right\rangle+p_{u}, \quad p_{u}:=\sum_{i, j=0}^{3}\left|\nabla_{v_{i}} \partial_{v_{j}} u\right|^{2} .
$$


We compute

$$
\begin{aligned}
\mathcal{L}^{\nabla} \partial_{v_{j}} u & =\sum_{i} \nabla_{v_{i}} \nabla_{v_{j}} \partial_{v_{i}} u-\sum_{i} \nabla_{v_{i}} \partial_{\left[v_{i}, v_{j}\right]} u \\
& =\sum_{i} R\left(\partial_{v_{i}} u, \partial_{v_{j}} u\right) \partial_{v_{i}} u+\nabla_{v_{j}} \mathcal{L}^{X} u-\sum_{i}\left(\nabla_{v_{i}} \partial_{\left[v_{i}, v_{j}\right]} u+\nabla_{\left[v_{i}, v_{j}\right]} \partial_{v_{i}} u\right) \\
& =\sum_{i} R\left(\partial_{v_{i}} u, \partial_{v_{j}} u\right) \partial_{v_{i}} u+h_{j}(u)+\xi_{j}(u),
\end{aligned}
$$

where the sums are over $i=0,1,2,3$ and

$$
\begin{aligned}
& h_{j}(u):=\left(\nabla_{v_{j}} \nabla_{S}-I \nabla_{v_{j}} \nabla_{v_{1}}-J \nabla_{v_{j}} \nabla_{v_{2}}-K \nabla_{v_{j}} \nabla_{v_{3}}\right) \nabla H(u), \\
& \xi_{j}(u):=-I \nabla_{v_{j}} \partial_{w_{1}} u-J \nabla_{v_{j}} \partial_{w_{2}} u-K \nabla_{v_{j}} \partial_{w_{3}} u-\sum_{i}\left(\nabla_{v_{i}} \partial_{\left[v_{i}, v_{j}\right]} u+\nabla_{\left[v_{i}, v_{j}\right]} \partial_{v_{i}} u\right) .
\end{aligned}
$$

Since the vector fields $v_{1}, v_{2}, v_{3}$ form an orthonormal frame of $T M$ there is a constant $c \geq 1$ such that, for every smooth map $u: \mathbb{R} \times M \rightarrow X$ and every smooth perturbation $H: X \times M \rightarrow \mathbb{R}$, we have

$$
\sum_{j=0}^{3}\left|\xi_{j}(u)\right|^{2} \leq c\left(e_{u}+p_{u}\right), \quad \sqrt{\sum_{j=0}^{3}\left|h_{j}(u)\right|^{2}} \leq c\|H\|_{C^{3}}\left(1+e_{u}+\sqrt{p_{u}}\right) .
$$

Here $p_{u}$ is as in (36). This gives

$$
\begin{aligned}
\left|\sum_{j=0}^{3}\left\langle\xi_{j}(u), \partial_{v_{j}} u\right\rangle\right| & \leq \frac{1}{2 c} \sum_{j=0}^{3}\left|\xi_{j}(u)\right|^{2}+\frac{c}{2} \sum_{j=0}^{3}\left|\partial_{v_{j}} u\right|^{2} \\
& \leq \frac{p_{u}}{2}+\left(c+\frac{1}{2}\right) e_{u}, \\
\left|\sum_{j=0}^{3}\left\langle h_{j}(u), \partial_{v_{j}} u\right\rangle\right| & \leq c\|H\|_{C^{3}} \sqrt{2 e_{u}}\left(1+e_{u}+\sqrt{p_{u}}\right) \\
& \leq \frac{p_{u}}{2}+c^{2}\|H\|_{C^{3}}^{2} e_{u}+\sqrt{2} c\|H\|_{C^{3}} \sqrt{e_{u}}\left(1+e_{u}\right) .
\end{aligned}
$$


Hence it follows from (36) that

$$
\begin{aligned}
\mathcal{L} e_{u}+r_{u} & =p_{u}+\sum_{j=0}^{3}\left\langle h_{j}(u)+\xi_{j}(u), \partial_{v_{j}} u\right\rangle \\
& \geq-\left(\frac{1}{2}+c+c^{2}\|H\|_{C^{3}}^{2}\right) e_{u}-\sqrt{2} c\|H\|_{C^{3}}\left(e_{u}\right)^{1 / 2}\left(1+e_{u}\right) \\
& \geq-c^{2}\|H\|_{C^{3}}^{2}-\left(1+c+c^{2}\|H\|_{C^{3}}^{2}\right) e_{u}-\sqrt{2} c\|H\|_{C^{3}}\left(e_{u}\right)^{3 / 2}
\end{aligned}
$$

and thus

$$
\mathcal{L} e_{u}+r_{u} \geq-C\left(\|H\|_{C^{3}}^{2}+\left(1+\|H\|_{C^{3}}^{2}\right) e_{u}+\|H\|_{C^{3}}\left(e_{u}\right)^{3 / 2}\right),
$$

where $C:=1+c^{2}$. Using the inequality $a b \leq \frac{1}{3} a^{3}+\frac{2}{3} b^{3 / 2}$ for $a, b \geq 0$ we obtain (34) with

$$
A:=C\left(\|H\|_{C^{3}}^{2}+\frac{1}{3}\left(1+\|H\|_{C^{3}}^{2}\right)^{3}\right), \quad B:=C\left(\frac{2}{3}+\|H\|_{C^{3}}\right) .
$$

This proves the lemma.

Remark 3.4 For general hyperkähler manifolds Lemma 3.3 gives an estimate of the form

$$
\mathcal{L} e \geq-c\left(1+e^{2}\right)
$$

for the energy density of solutions of (21) and (22). In dimensions $n=3,4$ the exponent 2 is larger than the critical exponent $(n+2) / n$ in Theorem B.1. For the critical points $f: M \rightarrow X$ of $\mathcal{A}_{H}$ this means that the energy

$$
\mathcal{E}(f)=\frac{1}{2} \int_{M}|d f|^{2} \operatorname{dvol}_{M}
$$

does not control the sup norm of $|d f|$ even if we assume that there is no energy concentration near points. This is related to noncompactness phenomena that can be easily observed in examples. Namely, composing a holomorphic sphere in $X$ (for one of the complex structures $J_{\lambda}=\lambda_{1} I+\lambda_{2} J+\lambda_{3} K$ ) with a suitable Hopf fibration gives rise to a solution of $\partial(f)=0$. Now the bubbling phenomenon for holomorphic spheres leads to sequences $f^{\nu}: S^{3} \rightarrow X$ of solutions of (21) where the derivative $d f^{v}$ blows up along a Hopf circle, while the energy remains bounded.

Lemma 3.5 There is a constant $C>0$, depending only on the vector fields $v_{i}$, the metric on $X$, and the Hamiltonian perturbation $H$, such that every solution $u$ : $\mathbb{R} \times M \rightarrow$ $X$ of (22) satisfies the estimate

$$
\mathcal{L}\left|\partial_{s} u\right|^{2} \geq-C\left(1+|d u|^{2}\right)\left|\partial_{s} u\right|^{2} .
$$


Proof Abbreviate $v_{0}:=\partial_{s}$ and $w_{1}:=\left[v_{2}, v_{3}\right], w_{2}:=\left[v_{3}, v_{1}\right], w_{3}:=\left[v_{1}, v_{2}\right]$ as in the proof of Lemma 3.3. Define the functions $e_{0}, r_{0}: \mathbb{R} \times M \rightarrow \mathbb{R}$ by

$$
e_{0}:=\frac{1}{2}\left|\partial_{s} u\right|^{2}, \quad r_{0}:=\sum_{i=1}^{3}\left\langle R\left(\partial_{s} u, \partial_{v_{i}} u\right) \partial_{v_{i}} u, \partial_{s} u\right\rangle
$$

Then

$$
\mathcal{L} e_{0}=\sum_{i=0}^{3}\left|\nabla_{v_{i}} \partial_{s} u\right|^{2}+\left\langle\mathcal{L}^{\nabla} \partial_{s} u, \partial_{s} u\right\rangle
$$

As in the proof of Lemma 3.3 we have

$$
\mathcal{L}^{\nabla} \partial_{s} u=\sum_{i} R\left(\partial_{v_{i}} u, \partial_{s} u\right) \partial_{v_{i}} u+h_{0}(u)+\xi_{0}(u)
$$

where the sum is over $i=1,2,3$ and

$$
\begin{aligned}
h_{0}(u) & :=\left(\nabla_{s} \nabla_{s}-I \nabla_{s} \nabla_{v_{1}}-J \nabla_{s} \nabla_{v_{2}}-K \nabla_{s} \nabla_{v_{3}}\right) \nabla H(u), \\
\xi_{0}(u): & :=-I \nabla_{s} \partial_{w_{1}} u-J \nabla_{s} \partial_{w_{2}} u-K \nabla_{s} \partial_{w_{3}} u .
\end{aligned}
$$

Now

$$
\left|h_{0}(u)\right|+\left|\xi_{0}(u)\right| \leq c\left(1+|d u|+\sqrt{\sum_{i}\left|\nabla_{v_{i}} \partial_{s} u\right|^{2}}\right)\left|\partial_{s} u\right|
$$

and hence it follows from (39) and (40) that

$$
\begin{aligned}
\mathcal{L} e_{0}+r_{0} & =\sum_{i}\left|\nabla_{v_{i}} \partial_{s} u\right|^{2}+\left\langle h_{0}(u)+\xi_{0}(u), \partial_{s} u\right\rangle \\
& \geq \sum_{i}\left|\nabla_{v_{i}} \partial_{s} u\right|^{2}-2 c\left(1+|d u|+\sqrt{\sum_{i}\left|\nabla_{v_{i}} \partial_{s} u\right|^{2}}\right) e_{0} \\
& \geq \frac{1}{2} \sum_{i}\left|\nabla_{v_{i}} \partial_{s} u\right|^{2}-2 c\left(1+|d u|+c e_{0}\right) e_{0} .
\end{aligned}
$$

Since $e_{0} \leq|d u|^{2}$ and $r_{0} \leq c|d u|^{2} e_{0}$ this proves (38).

\section{Compactness for critical points}

Theorem 3.6 Let $M$ be a Cartan hypercontact 3-manifold and $X$ be a compact flat hyperkähler manifold. Let $H: X \times M \rightarrow \mathbb{R}$ be any smooth function. Then the set of solutions of (21) is compact in the $C^{\infty}$ topology. 
Lemma 3.7 Let $M$ be a Cartan hypercontact 3-manifold and $X$ be a compact flat hyperkähler manifold. Then there is a constant $c>0$ such that

$$
\mathcal{A}(f) \leq c \int_{M}|\partial(f)|^{2} \mathrm{dvol}_{M}
$$

for every $f \in \mathcal{F}$. In particular, every solution of (13) is constant.

Proof Throughout we abbreviate

$$
\|f\|:=\sqrt{\int_{M}|f|^{2} \operatorname{dvol}_{M}}, \quad\|d f\|:=\sqrt{\int_{M}|d f|^{2} \operatorname{dvol}_{M} .}
$$

The Poincaré inequality asserts that there is a constant $C>0$ such that every smooth function $f: M \rightarrow \mathbb{H}^{n}$ satisfies

$$
\int_{M} f \mathrm{dvol}_{M}=0 \quad \Longrightarrow \quad\|f\| \leq C\|d f\| .
$$

Since $\alpha$ is a Cartan structure Equation (29) takes the form

$$
\not D D D f=d^{*} d f-\kappa \not D D f
$$

for $f: M \rightarrow \mathbb{H}^{n}$. Here we write $\not(f)=\not D D$ because $X=\mathbb{H}^{n}$ is equipped with the standard flat metric and $f \mapsto \partial(f)$ is a linear operator. Taking the inner product with $f$ we obtain

$$
\begin{aligned}
& \|d f\|^{2}=\int_{M}\langle f, \mathcal{D D D} f+\kappa \not D D f\rangle \operatorname{dvol}_{M} \\
& \leq\|\not D f\|^{2}+\kappa\|f\|\|\not D f\| \\
& \leq\|\not D f\|^{2}+\kappa C\|d f\|\|\mathbb{D} f\| \\
& \leq\|\not D f\|^{2}+\frac{1}{2}\|d f\|^{2}+\frac{\kappa^{2} C^{2}}{2}\|\not D f\|^{2}
\end{aligned}
$$

whenever $f$ has mean value zero. By Lemma 2.2, this implies

$$
\mathcal{A}(f)=\frac{1}{2}\left(\|d f\|^{2}-\|\not D f\|^{2}\right) \leq\left(1+\kappa^{2} C^{2}\right) \int_{M}|\not D f|^{2} \operatorname{dvol}_{M}
$$

for every smooth map $f: M \rightarrow \mathbb{H}^{n}$. (We can drop the mean value zero condition by adding a constant to $f$.) Now the theorem of Geiges-Gonzalo [13] shows that $M$ is a quotient of the 3-sphere by a finite subgroup of SU(2). If $M=S^{3}$ every smooth map $f: M \rightarrow X$ factors through a map to the universal cover $\mathbb{H}^{n}$ of $X$ and the assertion follows. The general case follows from the special case for the induced map on the universal cover of $M$. 
Proof of Theorem 3.6 By Lemma 3.7 the critical points of $\mathcal{A}_{H}$ satisfy a uniform action bound. The action bound and the energy identity of Lemma 2.2 give a uniform $L^{1}$ bound on the functions $e_{v}:=\left|d f_{v}\right|^{2}$. Since the exponent $\frac{3}{2}$ in the estimate (34) of Lemma 3.3 is less than the critical exponent $\frac{5}{3}$ we obtain from the Heinz trick (Theorem B.1) a uniform $L^{\infty}$ bound on the sequence $e_{v}$. Hence the result follows from Theorem 3.2.

Remark 3.8 If $M$ is the 3-torus then the assertion of Lemma 3.7 continues to hold for the contractible maps $f: M \rightarrow X$. In the noncontractible case we may have nonconstant solutions of (21) and the estimate of Lemma 3.7 only holds with an additional constant on the right.

Remark 3.9 Let $X$ be a $\mathrm{K} 3$ surface. Then compactness fails for the critical points of $\mathcal{A}_{H}$ even in the case $H=0$ and for sequences with bounded energy (see Remark 3.4).

\section{Compactness and exponential decay for Floer trajectories}

Lemma 3.10 Let $M$ be a Cartan hypercontact 3-manifold and $X$ be a compact hyperkähler manifold. Let $H: X \times M \rightarrow \mathbb{R}$ be any smooth function and $u: \mathbb{R} \times M \rightarrow X$ be a solution of (22). Then the following holds.

(i) For every $s \in \mathbb{R}$ we have

$$
\frac{1}{2} \int_{M}|d u|^{2} \leq \mathcal{A}(u(s, \cdot))+\operatorname{Vol}(M) \sup _{X \times M}|\nabla H|^{2}+\frac{3}{2} \int_{M}\left|\partial_{s} u\right|^{2} .
$$

(ii) If $u$ has finite energy

$$
\mathcal{E}_{H}(u)=\int_{-\infty}^{\infty} \int_{M}\left|\partial_{s} u\right|^{2} \kappa \operatorname{dvol}_{M} d s<\infty
$$

and $\sup |d u|<\infty$ then all the derivatives of $u$ are bounded on $\mathbb{R} \times M$ and $\partial_{s} u$ converges to zero in the $C^{\infty}$ topology as $s$ tends to $\pm \infty$.

(iii) If $X$ is flat then

$$
\mathcal{E}_{H}(u)<\infty \quad \Longrightarrow \quad \sup |d u|<\infty .
$$

Proof We prove (i). By Lemma 2.2, every solution $u$ of (22) satisfies

$$
\mathcal{E}(u(s, \cdot))=\mathcal{A}(u(s, \cdot))+\frac{1}{2} \int_{M}\left|\nabla H(u)-\partial_{s} u\right|^{2}
$$


and hence

$$
\begin{aligned}
\frac{1}{2} \int_{M}|d u|^{2} & =\mathcal{E}(u(s, \cdot))+\frac{1}{2} \int_{M}\left|\partial_{s} u\right|^{2} \\
& \leq \mathcal{A}(u(s, \cdot))+\operatorname{Vol}(M) \sup _{X \times M}|\nabla H|^{2}+\frac{3}{2} \int_{M}\left|\partial_{s} u\right|^{2}
\end{aligned}
$$

Here we have used the fact that the hypercontact structure on $M$ is a Cartan structure. This proves (i).

We prove (ii). Since $u$ satisfies (35) and $|d u|$ is bounded the standard elliptic bootstrapping arguments as in the proof of Theorem 3.1 give uniform bounds on the higher derivatives of $u$. Since $|d u|$ is bounded it follows from Lemma 3.5 that the function $\left|\partial_{s} u\right|^{2}$ satisfies an estimate of the form

$$
\mathcal{L}\left|\partial_{s} u\right|^{2} \geq-C\left|\partial_{S} u\right|^{2} .
$$

This in turn implies that $u$ satisfies the mean value inequality

$$
\left|\partial_{s} u\left(s_{0}, y\right)\right|^{2} \leq c \int_{s_{0}-1}^{s_{0}+1} \int_{M}\left|\partial_{s} u\right|^{2} \operatorname{dvol}_{M} d s
$$

for a suitable constant $c>0$ (see Theorem B.1 with $A=0$ and $\mu=\alpha=1$ ). Using the finite energy condition again we find that $\partial_{s} u$ converges to zero uniformly as $|s|$ tends to infinity. Convergence of the higher derivatives of $\partial_{s} u$ follows from an elliptic bootstrapping argument using Equation (40). This proves (ii).

We prove (iii). Assume $X$ is flat. Then it follows from Lemma 3.3 that there are positive constants $A$ and $B$ such that

$$
\mathcal{L}|d u|^{2} \geq-A-B|d u|^{3}
$$

for every solution $u: \mathbb{R} \times M \rightarrow X$ of (22). Hence, by Theorem B.1, there are positive constants $\hbar$ and $c$ such that every solution of (22) satisfies

$$
B^{2} \int_{B_{r}(z)}|d u|^{2}<\hbar \quad \Longrightarrow \quad|d u(z)|^{2} \leq c\left(A r^{2}+\frac{1}{r^{4}} \int_{B_{r}(z)}|d u|^{2}\right)
$$

for $z \in \mathbb{R} \times M$ and $0<r \leq 1$. Now suppose $u$ : $\mathbb{R} \times M \rightarrow X$ is a solution of (22) with finite energy $\mathcal{E}_{H}(u)<\infty$. Then the formula

$$
\int_{s_{0}}^{s_{1}} \int_{M}\left|\partial_{s} u\right|^{2} \kappa \operatorname{dvol}_{M} d s=\mathcal{A}_{H}\left(u\left(s_{0}, \cdot\right)\right)-\mathcal{A}_{H}\left(u\left(s_{1}, \cdot\right)\right)
$$

shows that there is a constant $C>0$ such that $\mathcal{A}_{H}(u(s, \cdot)) \leq C$ for all $s$. Explicitly we can choose $C:=\mathcal{A}(u(0, \cdot))+\mathcal{E}_{H}(u)$. Combining this with (43) we obtain an 
inequality

$$
\int_{M}|d u|^{2} \leq c+3 \int_{M}\left|\partial_{S} u\right|^{2}
$$

for every $s \in \mathbb{R}$, where $c:=2 C+2 \operatorname{Vol}(M) \sup |\nabla H|$. Next we choose $T>0$ so large that

$$
\int_{T}^{\infty} \int_{M}\left|\partial_{S} u\right|^{2} \operatorname{dvol}_{M}<\frac{\hbar}{4 B^{2}} .
$$

Then, for $z_{0}=\left(s_{0}, y_{0}\right) \in[T+1, \infty) \times M$ and $r<\hbar /\left(8 c B^{2}\right)$, we have

$$
\begin{aligned}
\int_{B_{r}\left(z_{0}\right)}|d u|^{2} & \leq \int_{s_{0}-r}^{s_{0}+r} \int_{M}|d u|^{2} \operatorname{dvol}_{M} d s \\
& \leq \int_{s_{0}-r}^{s_{0}+r}\left(c+3 \int_{M}\left|\partial_{s} u\right|^{2} \operatorname{dvol}_{M}\right) d s \\
& \leq 2 c r+3 \int_{T}^{\infty} \int_{M}\left|\partial_{s} u\right|^{2} \operatorname{dvol}_{M} d s \\
& \leq 2 c r+\frac{3 \hbar}{4 B^{2}}<\frac{\hbar}{B^{2}} .
\end{aligned}
$$

Here the second inequality follows from (45) and the third from the fact that $s_{0}-r>T$. The same estimate holds for $s_{0} \leq-T-1$. Hence it follows from (44) that $|d u|$ is bounded. This proves the lemma.

Remark 3.11 It is an open question if part (iii) of Lemma 3.10 continues to hold without the hypothesis that $X$ is flat.

Lemma 3.12 Let $M$ be a Cartan hypercontact 3-manifold and $X$ be a compact flat hyperkähler manifold. Let $H: X \times M \rightarrow \mathbb{R}$ be any smooth function. Then there is a constant $c>0$ such that

$$
-c \leq \mathcal{A}_{H}(u(s, \cdot)) \leq c
$$

for every finite energy solution $u$ : $\mathbb{R} \times M \rightarrow X$ of (22) and every $s \in \mathbb{R}$.

Proof By Theorem 3.6, there is a constant $c>0$ such that

$$
-c \leq \mathcal{A}_{H}(f) \leq c
$$

for every critical point of $\mathcal{A}_{H}$. Now let $u$ : $\mathbb{R} \times M \rightarrow X$ be a finite energy solution of (22) and choose a sequence of real numbers $s^{\nu} \rightarrow-\infty$. Passing to a subsequence we may assume that $u\left(s^{\nu}+\cdot, \cdot\right)$ converges, uniformly with all derivatives, to a solution 
of (22) on the domain $[-1,1] \times M$. By (i), this solution is a critical point of $\mathcal{A}_{H}$. Hence

$$
\lim _{\nu \rightarrow \infty} \mathcal{A}_{H}\left(u\left(s^{\nu}, \cdot\right)\right) \leq c
$$

Since the action is nonincreasing along negative gradient flow lines this shows that $\mathcal{A}(u(s, \cdot)) \leq c$ for all $s \in \mathbb{R}$. The lower bound is obtained by the same argument for a sequence $s^{v} \rightarrow+\infty$. This proves the lemma.

Theorem 3.13 (Exponential decay) Let $M$ be a Cartan hypercontact 3-manifold and $X$ be a compact hyperkähler manifold. Let $H: X \times M \rightarrow \mathbb{R}$ be a smooth function such that every solution of (21) is nondegenerate. Let $u: \mathbb{R} \times M \rightarrow X$ be a solution of (22). Then the following are equivalent.

(a) The energy $\mathcal{E}_{H}(u)$ is finite and $|d u|$ is bounded.

(b) There are solutions $f^{ \pm}: M \rightarrow X$ of Equation (21) such that

$$
\lim _{s \rightarrow \pm \infty} u(s, y)=f^{ \pm}(y), \quad \lim _{s \rightarrow \pm \infty} \mathcal{A}_{H}(u(s, \cdot))=\mathcal{A}_{H}\left(f^{ \pm}\right),
$$

and $\lim _{s \rightarrow \pm \infty} \partial_{s} u(s, y)=0$, Moreover, the convergence is uniform in $y$ and $|d u|$ is bounded.

(c) There are positive constants $\rho$ and $c_{1}, c_{2}, c_{3}, \ldots$ such that

$$
\left\|\partial_{s} u\right\|_{C} \ell((\mathbb{R} \backslash[-T, T]) \times M) \leq c_{\ell} e^{-\rho T}
$$

for every $T>0$ and every integer $\ell \geq 0$. Moreover, $|d u|$ is bounded.

Proof That (c) implies (a) is obvious. We prove that (a) implies (b). By Lemma 3.10 it follows from (a) that $\left|\partial_{s} u\right|$ converges to zero uniformly as $|s|$ tends to infinity and that $d u$ is uniformly bounded with all its derivatives. Hence every sequence $s_{v} \rightarrow \pm \infty$ has a subsequence, still denoted by $s_{v}$, such that $u\left(s_{v}, \cdot\right)$ converges in the $C^{\infty}$ topology to a solution of (21). Now it follows from the nondegeneracy of the critical points of $\mathcal{A}_{H}$ that they are isolated. Hence the limit is independent of the sequence $s_{v}$. This proves (b).

We prove that (b) implies (c). Consider the function $\phi: \mathbb{R} \rightarrow[0, \infty)$ defined by

$$
\phi(s):=\frac{1}{2} \int_{M} \kappa\left|\partial_{s} u\right|^{2} \operatorname{dvol}_{M} .
$$

By assumption, this function converges to zero as $s$ tends to $\pm \infty$. Moreover, its second derivative is given by

$$
\phi^{\prime \prime}(s)=\int_{M} \kappa\left|\nabla_{s} \partial_{s} u\right|^{2}+\int_{M} \kappa\left\langle\nabla_{s} \nabla_{s} \partial_{s} u, \partial_{s} u\right\rangle
$$


Denote by

$$
\mathbb{D}_{H}:=I \nabla_{v_{1}}+J \nabla_{v_{2}}+K \nabla_{v_{3}}-\nabla \nabla H(u)
$$

the covariant Hessian as in (24). Since the vector fields $v_{i}$ are independent of $s$ we have

$$
\nabla_{s} \partial_{s} u=-\nabla_{s} \partial_{H}(u)=-\mathbb{D}_{H} \partial_{s} u=\not_{H} \partial_{H}(u) .
$$

Differentiating this equation covariantly with respect to $s$ we obtain

$$
\nabla_{s} \nabla_{s} \partial_{s} u=\mathbb{D}_{H} \nabla_{s} \partial_{H}(u)+\left[\nabla_{s}, \mathbb{D}_{H}\right] \partial_{H}(u)=\mathbb{D}_{H} \mathcal{D}_{H} \partial_{s} u-\left[\nabla_{s}, \mathbb{D}_{H}\right] \partial_{s} u .
$$

Since $\mathbb{D}_{H}$ is self-adjoint with respect to the $L^{2}$ inner product with weight $\kappa$ this gives

$$
\phi^{\prime \prime}(s)=\int_{M} \kappa\left|\nabla_{s} \partial_{s} u\right|^{2}+\int_{M} \kappa\left|D_{D_{H}} \partial_{s} u\right|^{2}-\int_{M} \kappa\left\langle\left[\nabla_{s}, D_{H}\right] \partial_{s} u, \partial_{s} u\right\rangle .
$$

Since $|d u|$ is bounded we have an inequality

$$
\int_{M} \kappa\left\langle\left[\nabla_{s}, D_{H}\right] \partial_{s} u, \partial_{s} u\right\rangle \leq c\left\|\partial_{s} u\right\|_{L^{\infty}(M)} \int_{M}\left|\partial_{s} u\right|^{2} .
$$

Moreover, by Lemma 3.10, the bound on $|d u|$ guarantees that $u(s, \cdot)$ converges in the $C^{\infty}$ topology to $f^{ \pm}$as $s$ tends to $\pm \infty$. Since $f^{ \pm}$are nondegenerate critical points of $\mathcal{A}_{H}$ we deduce that there is a constant $\rho>0$ such that, for $|s|$ sufficiently large, we have

$$
\int_{M} \kappa\left|D_{H} \partial_{s} u\right|^{2} \geq 2 \rho^{2} \int_{M}\left|\partial_{s} u\right|^{2}
$$

Choosing $|s|$ so large that $c\left\|\partial_{s} u\right\|_{L^{\infty}(M)}<\rho^{2}$ we then obtain

$$
\phi^{\prime \prime}(s) \geq \rho^{2} \phi(s) \text {. }
$$

Hence $\quad \frac{d}{d s} e^{-\rho s}\left(\phi^{\prime}(s)+\rho \phi(s)\right)=e^{-\rho s}\left(\phi^{\prime \prime}(s)-\rho^{2} \phi(s)\right) \geq 0$.

Since $\phi(s) \rightarrow 0$ as $s \rightarrow \infty$ we must have

$$
\rho \phi(s)+\phi^{\prime}(s) \leq 0
$$

for all sufficiently large $s$ and hence $e^{\rho s} \phi(s)$ is nonincreasing. This proves the exponential decay for $\phi$. To establish exponential decay for the higher derivatives one can use an elliptic bootstrapping argument based on Equation (40) to show that the $L^{\infty}$ norm of $\partial_{s} u$ controls the higher derivatives. This proves the theorem.

Remark 3.14 If $X$ is flat then the condition sup $|d u|<\infty$ in (a)-(c) in Theorem 3.13 can be dropped. This follows from Lemma 3.10 (iii) and the fact that each of the conditions (46) and (47) guarantees finite energy. Similarly, the next theorem continues to hold for general compact hyperkähler manifolds if we impose the additional condition $\sup _{v} \sup _{\mathbb{R} \times M}\left|d u^{v}\right|<\infty$. 
Theorem 3.15 (Compactness) Let $M$ be a Cartan hypercontact 3-manifold and $X$ be a compact flat hyperkähler manifold. Let $H: X \times M \rightarrow \mathbb{R}$ be a smooth function such that every solution $f$ of $\partial_{H}(f)=0$ is nondegenerate. Let $f^{ \pm}$be two distinct critical points of $\mathcal{A}_{H}$ and $u^{v}$ be a sequence in $\mathcal{M}\left(f^{-}, f^{+} ; H\right)$. Then there is a subsequence (still denoted by $u^{v}$ ), a catenation

$$
u_{1} \in \mathcal{M}\left(f_{0}, f_{1} ; H\right), u_{2} \in \mathcal{M}\left(f_{1}, f_{2} ; H\right), \ldots, u_{N} \in \mathcal{M}\left(f_{N-1}, f_{N} ; H\right)
$$

of Floer trajectories, and there are sequences $s_{1}^{\nu}<s_{2}^{\nu}<\cdots<s_{N}^{v}$ such that

$$
f_{0}=f^{-}, \quad f_{N}=f^{+}, \quad \mathcal{A}_{H}\left(f_{j-1}\right)>\mathcal{A}_{H}\left(f_{j}\right),
$$

and, for $j=1, \ldots, N$, the shifted sequence $u^{v}\left(s_{j}^{v}+\cdot, \cdot\right)$ converges to $u_{j}$ uniformly with all derivatives on every compact subset of $\mathbb{R} \times M$.

Proof By Lemma 3.10 the functions $u^{v}$ satisfy (44) for suitable constants $A, B, c, \hbar$. This implies the following.

Energy quantization I Let $x_{0} \in \mathbb{R} \times M$ and suppose that there is a sequence $x^{v} \rightarrow x_{0}$ such that $\left|d u^{v}\left(x^{v}\right)\right|$ diverges to infinity. Then

$$
\liminf _{\nu \rightarrow \infty} \int_{B_{\varepsilon}\left(x_{0}\right)}\left|d u^{\nu}\right|^{2} \geq \frac{\hbar}{B^{2}}
$$

for every $\varepsilon>0$.

The proof uses the Wehrheim trick. Suppose, by contradiction, that there is a constant $\varepsilon>0$ and a sequence $v_{i} \rightarrow \infty$ such that $B^{2} \int_{B_{\varepsilon}\left(x_{0}\right)}\left|d u^{v_{i}}\right|^{2}<\hbar$ for every $i$. Then we can use (44) with $x \in B_{\varepsilon / 2}\left(x_{0}\right)$ and $r=\varepsilon / 2$ to obtain

$$
\left|d u^{v_{i}}(x)\right|^{2} \leq c\left(\frac{A \varepsilon^{2}}{4}+\frac{16}{\varepsilon^{4}} \int_{B_{\varepsilon}\left(x_{0}\right)}\left|d u^{v_{i}}\right|^{2}\right) \leq \frac{A c \varepsilon^{2}}{4}+\frac{16 c \hbar}{B^{2} \varepsilon^{4}}
$$

for all $x \in B_{\varepsilon / 2}\left(x_{0}\right)$ and $v \geq v_{0}$. With $x=x^{v_{i}}$ it follows that the sequence $\left|d u^{v_{i}}\left(x^{\nu_{i}}\right)\right|$ is bounded, a contradiction.

Energy quantization II Let $x_{0}=\left(s_{0}, y_{0}\right) \in \mathbb{R} \times M$ and suppose that there is a sequence $x^{v}=\left(s^{\nu}, y^{\nu}\right) \rightarrow\left(s_{0}, x_{0}\right)$ such that $\left|d u^{v}\left(x^{\nu}\right)\right|$ diverges to infinity. Then

$$
\liminf _{\nu \rightarrow \infty} \int_{s_{0}-\varepsilon}^{s_{0}+\varepsilon} \int_{M}\left|\partial_{s} u^{v}\right|^{2} \geq \frac{\hbar}{3 B^{2}}
$$

for every $\varepsilon>0$. 
By Lemma 3.10 (i) we have

$$
\int_{M}\left|\partial_{s} u^{v}\right|^{2} \geq \frac{1}{3} \int_{M}\left|d u^{v}\right|^{2}-c
$$

for some constant $c>0$ independent of $v$ and $s$. The assertion follows by integrating this inequality from $s_{0}-\varepsilon$ to $s_{0}+\varepsilon$ and taking the limit $\varepsilon \rightarrow 0$.

With this understood it follows that, after passing to a subsequence, we obtain divergence of the energy density at most near finitely many points. On the complement of these finitely many points, a further subsequence converges to a solution $u^{\infty}$ of $\partial_{s} u^{\infty}+\partial_{H}\left(u^{\infty}\right)=0$ in the $C^{\infty}$ topology, by Theorem 3.2. Now it follows from the inequality (48) that the $L^{2}$ norm of $d u^{\infty}$ is finite on every compact subset of $\mathbb{R} \times M$ and in particular in a neighborhood of each bubbling point. Hence we can use the removable singularity Theorem C.1 to deduce that the limit solution can be extended into the finitely many missing points. The upshot is that, by standard arguments, we obtain a convergent subsequence as in the statement of the theorem, except that $u^{v}\left(s_{j}^{v}+\cdot, \cdot\right)$ need only converge to $u_{j}$ in the complement of finitely many points. If these bubbling points do exist we have

$$
\mathcal{A}_{H}\left(f_{j-1}\right)-\mathcal{A}_{H}\left(f_{j}\right)=\mathcal{E}_{H}\left(u_{j}\right) \leq \lim _{T \rightarrow \infty} \int_{s_{j}^{\nu}-T}^{s_{j}^{\nu}+T} \int_{M}\left|\partial_{s} u^{\nu}\right|^{2}-\frac{\hbar}{B^{2}}
$$

for some $j$. However, this would imply that the sum of the energies $\mathcal{E}_{H}\left(u_{j}\right)$ is strictly smaller than $\mathcal{E}_{H}\left(u^{v}\right)=\mathcal{A}_{H}\left(f^{-}\right)-\mathcal{A}_{H}\left(f^{+}\right)$which is clearly impossible. Thus bubbling cannot occur and the sequence $\left|d u^{v}\right|$ must remain uniformly bounded. This proves the theorem.

Remark 3.16 A key issue in developing the Floer theory of the action functional $\mathcal{A}_{H}$ for general (compact) hyperkähler manifolds is to extend Theorems 3.6 and 3.15 to the nonflat case. One then has to address the codimension- 2 bubbling phenomenon for finite energy sequences of solutions $f$ of $\partial_{H}(f)=0$ and $u$ of $\partial_{s} u+\partial_{H}(u)=0$.

\section{Moduli spaces and transversality}

\section{Transversality for critical points}

Let $\mathcal{H}:=C^{\infty}(X \times M)$ and, for $H \in \mathcal{H}$, denote by

$$
\mathcal{C}(H):=\left\{f: M \rightarrow X \mid f \text { satisfies } \partial_{H}(f)=0\right\}
$$


the set of critical points of $\mathcal{A}_{H}$. Recall that a critical point $f \in \mathcal{C}(H)$ is called nondegenerate if the Hessian

$$
\mathcal{D}_{f, H}:=I \nabla_{v_{1}}+J \nabla_{v_{2}}+K \nabla_{v_{3}}-\nabla \nabla H(f)
$$

is bijective as an operator from $T_{f} \mathcal{F}=\Omega^{0}\left(M, f^{*} T X\right)$ to itself (respectively as an operator from $W^{k+1, p}\left(M, f^{*} T X\right)$ to $\left.W^{k, p}\left(M, f^{*} T X\right)\right)$. Denote by

$$
\mathcal{H}^{\text {morse }}:=\{H \in \mathcal{H} \mid \text { every critical point } f \in \mathcal{C}(H) \text { is nondegenerate }\}
$$

of all $H \in \mathcal{H}$ such that $\mathcal{A}_{H}: \mathcal{F} \rightarrow \mathbb{R}$ is a Morse function.

Theorem 4.1 For every compact 3-manifold $M$ with a positive hypercontact structure and every hyperkähler manifold $X$ the set $\mathcal{H}^{\text {morse }}$ is of the second category in $\mathcal{H}$.

Proof Fix an integer $\ell \geq 2$ and abbreviate $\mathcal{H}^{\ell}:=C^{\ell}(X \times M)$. Then the regularity argument in the proof of Theorem 3.1 shows that $f$ with $\partial_{H}(f)=0$ is of class $W^{\ell, p}$ for any $p<\infty$. Fix a constant $p>3$ and denote by

$$
\mathcal{C}^{\ell}:=\left\{(f, H) \in W^{1, p}(M, X) \times \mathcal{H}^{\ell} \mid f \text { satisfies } \partial_{H}(f)=0\right\}
$$

the universal moduli space of critical points. We prove that $\mathcal{C}^{\ell}$ is a $C^{\ell-1}$ Banach manifold. It is the zero set of a $C^{\ell-1}$ section of a Banach space bundle

$$
\mathcal{E} \rightarrow W^{1, p}(M, X) \times \mathcal{H}^{\ell}
$$

with fibers $\mathcal{E}_{f, H}:=L^{p}\left(M, f^{*} T x\right)$. The section is given by

$$
(f, H) \mapsto \partial_{H}(f)
$$

and we must prove that it is transverse to the zero section. Equivalently, the operator

$$
W^{1, p}\left(M, f^{*} T X\right) \times \mathcal{H}^{\ell} \rightarrow L^{p}\left(M, f^{*} T X\right),(\xi, h) \mapsto \mathbb{D}_{f, H} \xi-\nabla h(f)
$$

is surjective for every $H \in \mathcal{H}^{\ell}$ and every $f \in \mathcal{C}(H)$.

Let $1 / p+1 / q=1$ and choose an element $\eta \in L^{q}\left(M, f^{*} T X\right)$ that annihilates the image of (49) in the sense that

$$
\int_{M}\left\langle\eta, D_{f, H} \xi-\nabla h(f)\right\rangle \kappa \operatorname{dvol}_{M}=0
$$

for all $h \in \mathcal{H}_{\ell}$ and $\xi \in W^{1, p}\left(M, f^{*} T X\right)$. Then, by elliptic regularity, we have $\eta \in W^{\ell, p}\left(M, f^{*} T X\right)$ and

$$
\mathbb{D}_{f, H} \eta=0, \quad \int_{M}\langle\eta, \nabla h(f)\rangle \kappa \operatorname{dvol}_{M}=0 \quad \forall h \in \mathcal{H}^{\ell} .
$$


In particular $\eta$ is continuous. If $\eta \not \equiv 0$ then it is easy to find a smooth function $h: X \times M \rightarrow \mathbb{R}$ such that $\langle\eta, \nabla h(f)\rangle \geq 0$ everywhere on $M$ and $\langle\eta, \nabla h(f)\rangle>0$ somewhere. Namely, choose a point $y_{0} \in M$ with $\eta\left(y_{0}\right) \neq 0$ and a function $h_{0}: X \rightarrow \mathbb{R}$ such that

$$
h_{0}\left(f\left(y_{0}\right)\right)=0, \quad \nabla h_{0}\left(f\left(y_{0}\right)\right)=\eta\left(y_{0}\right) .
$$

Then there is a neighborhood $U_{0} \subset M$ of $y_{0}$ such that

$$
\left\langle\eta(y), \nabla h_{0}(f(y))\right\rangle>0
$$

for all $y \in U_{0}$. Now choose a smooth cutoff function $\beta: M \rightarrow[0,1]$ with support in $U_{0}$ such that $\beta\left(y_{0}\right)=1$. Then the function $h(y, x):=\beta(y) h_{0}(x)$ has the required properties. Thus we have proved that the operator (49) is always surjective and hence $\mathcal{C}^{\ell}$ is a $C^{\ell-1}$ Banach manifold as claimed.

Now the obvious projection

$$
\pi^{\ell}: \mathcal{C}^{\ell} \rightarrow \mathcal{H}^{\ell}
$$

is a $C^{\ell-1}$ Fredholm map of index zero. Since $\ell \geq 2$, it follows from the Sard-Smale theorem that the set $\mathcal{H}^{\text {morse, } \ell} \subset \mathcal{H}^{\ell}$ of regular values of $\pi^{\ell}$ is dense in $\mathcal{H}^{\ell}$. Now the result follows by the usual Taubes trick as explained, for example, in [22, Chapter 3]. Namely, for a constant $c>0$ we may introduce the set $\mathcal{H}_{c}^{\text {morse, } \ell}$ of all $H \in \mathcal{H}^{\ell}$ such that the critical points $f \in \mathcal{C}(H)$ with $\sup |d f| \leq c$ are nondegenerate. By Theorem 3.2, this set is open in $\mathcal{H}^{\ell}$. (In fact Theorem 3.2 can be extended to obtain a $W^{\ell-1, p}$ convergent subsequence whenever $H$ is of class $C^{\ell}$ respectively converges in $C^{\ell}$.) Since $\mathcal{H}^{\text {morse }, \ell}=\bigcap_{c>0} \mathcal{H}_{c}^{\text {morse, } \ell}$, we obtain with $\ell=\infty$ that each corresponding set $\mathcal{H}_{c}^{\text {morse }}$ is open and dense in $\mathcal{H}$ and so $\mathcal{H}^{\text {morse }}=\bigcap_{c>0} \mathcal{H}_{c}^{\text {morse }}$ is a countable intersection of open and dense sets in $\mathcal{H}$. This proves the theorem.

\section{Fredholm theory}

The study of the spaces of solutions of (22) is based on the linearized operators $\mathcal{D}_{u, H}: W^{1, p}\left(\mathbb{R} \times M, u^{*} T X\right) \rightarrow L^{p}\left(\mathbb{R} \times M, u^{*} T X\right)$ defined by

$$
\mathcal{D}_{u, H}:=\nabla_{s}+I \nabla_{v_{1}}+J \nabla_{v_{2}}+K \nabla_{v_{3}}-\nabla \nabla H(u) .
$$

It follows from the familiar arguments in Floer homology that $\mathcal{D}_{u, H}$ is a Fredholm operator whenever $f^{ \pm}$are nondegenerate critical points of $\mathcal{A}_{H}$ and $u$ satisfies the exponential decay conditions of Theorem 3.13. It is also a standard result that the Fredholm index of $\mathcal{D}_{u, H}$ is given by the spectral flow of Atiyah-Patodi-Singer [2]. More precisely, given a contractible critical point $f \in \mathcal{C}(H)$ choose a smooth path $[0,1] \rightarrow \mathcal{F}: t \mapsto f_{t}$ such that

$$
f_{0} \equiv \text { constant }, \quad f_{1}=f
$$


and choose $\varepsilon>0$ such that the negative eigenvalues of $\mathcal{D}_{f_{0}}$ are all less than $-\varepsilon$. Now define the integer $\mu_{H}(f)$ by the formula

$$
\mu_{H}(f):=-\operatorname{specflow}\left(\left\{\mathbb{D}_{f_{t}, t H}+\varepsilon(1-t) \mathbb{1}\right\}_{0 \leq t \leq 1}\right) .
$$

It follows from Equation (11) (with $\Sigma=S^{1} \times M$ ) that this integer is independent of homotopy $t \mapsto f_{t}$ whenever $X$ is flat. If $f: M \rightarrow X$ is not contractible then the definition of the index $\mu_{H}(f)$ depends on the choice of a fixed reference map $f_{0}$.

Proposition 4.2 Assume $H \in \mathcal{H}^{\text {morse }}$ and $f^{ \pm} \in \mathcal{C}(H)$.

(i) For every smooth map $u: \mathbb{R} \times M \rightarrow X$ satisfying (23) the operator

$$
\mathcal{D}_{u, H}: W^{1, p}\left(\mathbb{R} \times M, u^{*} T M\right) \rightarrow L^{p}\left(\mathbb{R} \times M, u^{*} T M\right)
$$

is Fredholm and its Fredholm index is

$$
\operatorname{index}\left(\mathcal{D}_{u, H}\right)=\mu_{H}\left(f^{-}\right)-\mu_{H}\left(f^{+}\right) .
$$

(ii) If $H: X \rightarrow \mathbb{R}$ is a Morse function with sufficiently small $C^{2}$ norm and $f(y) \equiv$ $x_{0}$ is a critical point of $H$ then $\mu_{H}(f)=\operatorname{dim} X-\operatorname{ind}_{H}\left(x_{0}\right)$ is equal to the Morse index of $x_{0}$ as a critical point of $-H$ (ie the number of positive eigenvalues of the Hessian of $H$ at $x_{0}$ ).

Proof The Fredholm property in (i) follows from standard arguments in Floer theory as in Donaldson [6] and Floer [9] in the instanton setting and in Floer [10] and Salamon [27] in the symplectic setting. The index identity is a well known result about the correspondence between the spectral flow and the Fredholm index (see Atiyah-Patodi-Singer [2] and Robbin-Salamon [25]). The second assertion follows immediately from the definition of $\mu_{H}$.

\section{Transversality for Floer trajectories}

For $f^{ \pm} \in \mathcal{C}(H)$ we denote by $\mathcal{M}\left(f^{-}, f^{+} ; H\right)$ the moduli space of all solutions $u: \mathbb{R} \times M \rightarrow X$ of (22) and (23) for which $|d u|$ is bounded. To prove that these spaces are smooth manifolds we must show that the linearized operator $\mathcal{D}_{u, H}$ is surjective for every solution $u$ of Equation (22) and (23). Let

$$
\mathcal{H}^{\text {reg }} \subset \mathcal{H}
$$

denote the set of all Hamiltonian perturbations $H \in \mathcal{H}$ such that $\mathbb{D}_{f, H}$ is bijective for every critical point $f \in \mathcal{C}(H)$ of $\mathcal{A}_{H}$ and $\mathcal{D}_{u, H}$ is surjective for every $u \in \mathcal{M}\left(f^{-}, f^{+} ; H\right)$ and all $f^{ \pm} \in \mathcal{C}(H)$. 
Theorem 4.3 For every compact 3-manifold $M$ with a positive hypercontact structure and every hyperkähler manifold $X$ the set $\mathcal{H}^{\text {reg }}$ is of the second category in $\mathcal{H}$. If $H \in \mathcal{H}^{\text {reg }}$ then the moduli space $\mathcal{M}\left(f^{-}, f^{+} ; H\right)$ is a smooth manifold of dimension

$$
\operatorname{dim} \mathcal{M}\left(f^{-}, f^{+} ; H\right)=\mu_{H}\left(f^{-}\right)-\mu_{H}\left(f^{+}\right)
$$

for every pair $f^{ \pm} \in \mathcal{C}(H)$.

To prove this result we follow essentially the discussion in [12]. The first step is a unique continuation result.

Proposition 4.4 Let $\ell \geq 3, H \in \mathcal{H}^{\ell}$, and $u_{0}, u_{1}: \mathbb{R} \times M \rightarrow X$ be two $C^{\ell-1}$ solution of (22). If $u_{0}$ and $u_{1}$ agree to infinite order at a point $\left(s_{0}, y_{0}\right) \in \mathbb{R} \times M$ then they agree everywhere.

Proof In local coordinates $x^{1}, \ldots, x^{m}$ on $X$ and $y^{1}, y^{2}, y^{3}$ on $M$ both functions satisfy Equation (32). For the difference

$$
\widehat{u}^{k}:=\left(u_{1}-u_{0}\right)^{k}
$$

in local coordinates this gives an estimate

$$
\left|\mathcal{L} \widehat{u}^{k}\right| \leq c \sum_{j=1}^{m}\left(\left|\hat{u}^{j}\right|+\left|\frac{\partial \widehat{u}^{j}}{\partial s}\right|+\sum_{\nu=1}^{3}\left|\frac{\partial \widehat{u}^{j}}{\partial y^{v}}\right|\right), \quad k=1, \ldots, m .
$$

This is precisely the hypothesis of Aronszajn's theorem [1]. Hence, if $\hat{u}$ vanishes to infinite order at a point it must vanish identically in a neighborhood of that point. This implies that the set of all points $(s, y)$ where $u_{0}$ and $u_{1}$ agree to infinite order is open and closed. This proves the proposition.

Proposition 4.5 Let $H \in \mathcal{H}$ and $u: \mathbb{R} \times M \rightarrow X$ be a smooth map. Let $\xi \in$ $\Omega^{0}\left(\mathbb{R} \times M, u^{*} T X\right)$ be a vector field along $u$ such that

$$
\mathcal{D}_{u, H} \xi=\nabla_{s} \xi+I \nabla_{v_{1}} \xi+J \nabla_{v_{2}} \xi+K \nabla_{v_{3}} \xi-\nabla_{\xi} \nabla H(u)=0 .
$$

If $\xi \not \equiv 0$ then the set

$$
\mathcal{Z}:=\{(s, y) \in \mathbb{R} \times M \mid \xi(s, y)=0\}
$$

can be covered by countably many codimension 2 submanifolds of $\mathbb{R} \times M$. In particular, the set $(\mathbb{R} \times M) \backslash \mathcal{Z}$ is open, connected, and dense in $\mathbb{R} \times M$. 
Proof The proof has three steps.

Step 1 If $\xi$ vanishes to infinite order at a point $\left(s_{0}, y_{0}\right) \in \mathbb{R} \times M$ then $\xi$ vanishes identically.

We use the identity

$$
\begin{aligned}
\mathcal{D}^{*} \mathcal{D} \xi+\mathcal{L}^{\nabla} \xi= & -I\left(R\left(\partial_{s} u, \partial_{v_{1}} u\right) \xi-R\left(\partial_{v_{2}} u, \partial_{v_{3}} u\right) \xi+\nabla_{\left[v_{2}, v_{3}\right]} \xi\right) \\
& -J\left(R\left(\partial_{s} u, \partial_{v_{2}} u\right) \xi-R\left(\partial_{v_{3}} u, \partial_{v_{1}} u\right) \xi+\nabla_{\left[v_{3}, v_{1}\right]} \xi\right) \\
& -K\left(R\left(\partial_{s} u, \partial_{v_{3}} u\right) \xi-R\left(\partial_{v_{1}} u, \partial_{v_{2}} u\right) \xi+\nabla_{\left[v_{1}, v_{2}\right]} \xi\right) .
\end{aligned}
$$

If $\mathcal{D} \xi=\nabla_{\xi} \nabla H(u)$ we obtain an inequality of the form

$$
\left|\mathcal{L}^{\nabla} \xi\right| \leq c\left(|\xi|+\left|\nabla_{s} \xi\right|+\sum_{j=1}^{3}\left|\nabla_{v_{j}} \xi\right|\right) .
$$

In local coordinates the leading term of $\mathcal{L}^{\nabla}$ has diagonal form. Hence the assertion of Step 1 follows from Aronszajn's theorem [1].

Step 2 Let $\mathcal{Z}_{k} \subset \mathcal{Z}$ denote the set where $\xi$ and its derivatives vanish up to order $k$. Then, for every $z_{0}=\left(s_{0}, y_{0}\right) \in \mathcal{Z}_{k} \backslash \mathcal{Z}_{k+1}$, there is an open neighborhood $U_{0} \subset \mathbb{R} \times M$ and a codimension 2 submanifold $V \subset \mathbb{R} \times M$ such that

$$
\left(\mathcal{Z}_{k} \backslash \mathcal{Z}_{k+1}\right) \cap U_{0} \subset V .
$$

Fix an element $z_{0} \in \mathcal{Z}_{k} \backslash \mathcal{Z}_{k+1}$. For $v=\left(v_{0}, v_{1}, v_{2}, v_{3}\right) \in \mathbb{N}^{4}$ denote

$$
\nabla^{v} \xi:=\nabla_{v_{0}} \cdots \nabla_{v_{0}} \nabla_{v_{1}} \cdots \nabla_{v_{1}} \nabla_{v_{2}} \cdots \nabla_{v_{2}} \nabla_{v_{3}} \cdots \nabla_{v_{3}} \xi
$$

where $v_{0}:=\partial_{s}$ and each term $\nabla_{v_{i}}$ occurs $v_{i}$ times. Since all derivatives of $\xi$ vanish up to order $k$ at the point $z_{0}$ we have

$$
\nabla_{v_{i}} \nabla^{v} \xi\left(z_{0}\right)=\nabla^{v} \nabla_{v_{i}} \xi\left(z_{0}\right)
$$

for $|v|:=v_{0}+v_{1}+v_{2}+v_{3}=k$ and $i=0,1,2,3$. Since $z_{0} \notin \mathcal{Z}_{k+1}$ there is a multi-index $v \in \mathbb{N}^{4}$ with $|v|=k$ and an $i \in\{0,1,2,3\}$ such that $\nabla_{v_{i}} \nabla^{v} \xi\left(z_{0}\right) \neq 0$. Consider the vector field

$$
\eta:=\nabla^{v} \xi
$$

along $u$. Again using the fact that all derivatives of $\xi$ up to order $k$ vanish at $z_{0}$ we obtain

$$
\nabla_{v_{0}} \eta\left(z_{0}\right)+I \nabla_{v_{1}} \eta\left(z_{0}\right)+J \nabla_{v_{2}} \eta\left(z_{0}\right)+K \nabla_{v_{3}} \eta\left(z_{0}\right)=0 .
$$


Since one of the vectors $\nabla_{v_{i}} \eta\left(z_{0}\right)$ is nonzero it follows that the four vectors $\nabla_{v_{i}} \eta\left(z_{0}\right)$ cannot all be linearly dependent. Hence, in local coordinates $x^{1}, \ldots, x^{m}$ on $X$ there exist indices $i, j \in\{1, \ldots, m\}$ such that the differentials of the functions $\eta^{i}, \eta^{j}$ (on an open neighborhood of $z_{0}$ in $\left.\mathbb{R} \times M\right)$ are linearly independent. Hence, by the implicit function theorem, there is a neighborhood $U_{0} \subset \mathbb{R} \times M$ of $z_{0}$ such that the set

$$
V:=\left\{x \in U_{0} \mid \eta^{i}(x)=\eta^{j}(x)=0\right\}
$$

is a codimension 2 submanifold of $\mathbb{R} \times M$. Since

$$
\left(\mathcal{Z}_{k} \backslash \mathcal{Z}_{k+1}\right) \cap U_{0} \subset V
$$

this proves Step 2.

Step 3 We prove the proposition.

By Step 1 we have

$$
\mathcal{Z}=\bigcup_{k=0}^{\infty}\left(\mathcal{Z}_{k} \backslash \mathcal{Z}_{k+1}\right) .
$$

By Step 2 each of the sets $\mathcal{Z}_{k} \backslash \mathcal{Z}_{k+1}$ can be covered by finitely many submanifolds of codimension 2. This proves the proposition.

Let $H \in \mathcal{H}^{\ell}$ and $u: \mathbb{R} \times M \rightarrow X$ be a $C^{\ell-1}$ solution of (22) and (23). Call a point $(s, y) \in \mathbb{R} \times M$ regular if

$$
\partial_{s} u(s, y) \neq 0, \quad u(s, y) \neq f^{ \pm}(y), \quad u(s, y) \notin u(\mathbb{R} \backslash\{s\}, y) .
$$

Let $\mathcal{R}(u) \subset \mathbb{R} \times M$ denote the set of regular points of $u$.

Proposition 4.6 Fix an integer $\ell \geq 4$. Let $H \in \mathcal{H}^{\ell}$ and $u: \mathbb{R} \times M \rightarrow X$ be a $C^{\ell-1}$ solution of (22) and (23) with $f^{-} \neq f^{+}$. Then the set $\mathcal{R}(u)$ of regular points of $u$ is open and dense in $\mathbb{R} \times M$.

Proof That the set $\mathcal{R}(u)$ is open follows by the same argument as in the proof of [12, Theorem 4.3]. We prove in four steps that $\mathcal{R}(u)$ is dense.

Step 1 The set

$$
\mathcal{R}_{0}(u):=\left\{(s, y) \in \mathbb{R} \times M \mid \partial_{s} u(s, y) \neq 0\right\}
$$

is open and dense in $\mathbb{R} \times M$.

The vector field $\partial_{s} u$ is in the kernel of the linearized operator $\mathcal{D}_{u, H}$ and is a vector field of class $C^{\ell-2}$ and hence of class $C^{2}$. Now Step 1 in the proof of Proposition 4.5 
continues to hold for $C^{2}$ vector fields and hence the set $\mathcal{R}_{0}(u)$ is dense in $\mathbb{R} \times M$. That it is open is obvious. This proves Step 1.

\section{Step 2 The set}

$$
\mathcal{R}_{1}(u):=\left\{(s, y) \in \mathcal{R}_{0}(u) \mid u(s, y) \neq f^{ \pm}(y)\right\}
$$

is open and dense in $\mathbb{R} \times M$.

That the set is open is obvious. We prove it is dense. By Step 1 it suffices to prove that every point $(s, y) \in \mathcal{R}_{0}(u)$ can be approximated by a sequence in $\mathcal{R}_{1}(u)$. Because $\partial_{s} u(s, y) \neq 0$, every sequence $\left(s_{v}, y\right)$ with $s_{v} \rightarrow s$ and $s_{v} \neq s$ belongs to the set $\mathcal{R}_{1}(u)$ for $v$ sufficiently large. This proves Step 2.

Step 3 The set

$$
\mathcal{R}_{2}(u):=\left\{(s, y) \in \mathcal{R}_{1}(u) \mid u(s, y) \notin u\left(\mathbb{R} \times\{y\} \backslash \mathcal{R}_{0}(u)\right)\right\}
$$

is open and dense in $\mathbb{R} \times M$.

We prove that the set is open. Suppose, by contradiction, that there is an element $\left(s_{0}, y_{0}\right) \in \mathcal{R}_{2}(u)$ and a sequence $\left(s_{\nu}, y_{\nu}\right) \in \mathcal{R}_{1}(u) \backslash \mathcal{R}_{2}(u)$ converging to $\left(s_{0}, y_{0}\right)$. Since $\left(s_{v}, y_{v}\right) \notin \mathcal{R}_{2}(u)$ there is an $s_{v}^{\prime} \in \mathbb{R}$ such that

$$
\partial_{s} u\left(s_{v}^{\prime}, y_{v}\right)=0, \quad u\left(s_{v}^{\prime}, y_{v}\right)=u\left(s_{v}, y_{v}\right) .
$$

The sequence $s_{v}^{\prime}$ must be bounded; for if $s_{v}^{\prime} \rightarrow \pm \infty$ then $u\left(s_{v}^{\prime}, y_{v}\right)$ converges to $f^{ \pm}\left(y_{0}\right)$ and this implies $u\left(s_{0}, y_{0}\right)=f^{ \pm}\left(y_{0}\right)$, a contradiction. Thus, passing to a subsequence, we may assume that $s_{v}^{\prime}$ converges to a point $s_{0}^{\prime} \in \mathbb{R}$. It then follows that $u\left(s_{0}, y_{0}\right)=u\left(s_{0}^{\prime}, y_{0}\right)$ and $\partial_{s} u\left(s_{0}^{\prime}, y_{0}\right)=0$, contradicting the fact that $\left(s_{0}, y_{0}\right) \in \mathcal{R}_{2}(u)$.

We prove that the set $\mathcal{R}_{2}(u)$ is dense in $\mathbb{R} \times M$. It suffices to prove that every element $\left(s_{0}, y_{0}\right) \in \mathcal{R}_{1}(u)$ can be approximated by a sequence in $\mathcal{R}_{2}(u)$. If this is not the case for some element $\left(s_{0}, y_{0}\right) \in \mathcal{R}_{1}(u)$ then there is an $\varepsilon>0$ such that the following holds:

$$
\left|s-s_{0}\right|<\varepsilon \Longrightarrow \exists s^{\prime} \in \mathbb{R} \text { such that } u\left(s, y_{0}\right)=u\left(s^{\prime}, y_{0}\right), \partial_{s} u\left(s^{\prime}, y_{0}\right)=0 \text {. }
$$

However this contradicts Sard's theorem. Namely for $\varepsilon$ small the curve

$$
\Gamma:=\left\{u\left(s, y_{0}\right)|| s-s_{0} \mid<\varepsilon\right\}
$$

is a one dimensional submanifold of $X$ and we can choose a projection $\pi: U \rightarrow \Gamma$ on a suitable tubular neighborhood. Consider the open set $S:=\left\{s \in \mathbb{R} \mid u\left(s, y_{0}\right) \in U\right\}$. The assertion would then mean that every element of $\Gamma$ is a singular value of the map 
$S \rightarrow \Gamma: s \mapsto \pi\left(u\left(s, y_{0}\right)\right)$. By Sard's theorem, this is impossible whenever $u$ is $C^{1}$. This proves Step 3.

Step 4 The set $\mathcal{R}(u)$ is open and dense in $\mathbb{R} \times M$.

We have already observed that the set is open. We prove it is dense. By Step 3, it suffices to prove that every element of $\mathcal{R}_{2}(u)$ can be approximated by a sequence in $\mathcal{R}(u)$. Suppose, by contradiction, that this is not the case for some element $\left(s_{0}, y_{0}\right) \in \mathcal{R}_{2}(u)$. Then there is an open neighborhood $U \subset M$ of $y_{0}$ and two positive real number $\varepsilon, T$ such that the following holds. We abbreviate $I:=\left(s_{0}-\varepsilon, s_{0}+\varepsilon\right)$.

(a) $I \times U \subset \mathcal{R}_{2}(u) \backslash \mathcal{R}(u)$.

(b) $u(s, y) \notin u(I \times U)$ for $|s| \geq T$ and $y \in U$.

(c) The map $\bar{I} \rightarrow X: s \mapsto u(s, y)$ is an embedding for every $y \in U$.

Since $I \times U \subset \mathcal{R}_{1}(u)$, the condition $I \times U \cap \mathcal{R}(u)=\varnothing$ means that for every $(s, y) \in$ $I \times U$ there is an $s^{\prime} \in \mathbb{R} \backslash\{s\}$ such that $u\left(s^{\prime}, y\right)=u(s, y)$. Since $(s, y) \in \mathcal{R}_{2}(u)$ we must have $\partial_{s} u\left(s^{\prime}, y\right) \neq 0$ and, by (b), we have $\left|s^{\prime}\right| \leq T$. Hence there can only be finitely many such points $s^{\prime}$. For $s=s_{0}$ let $s_{1}<\cdots<s_{N}$ be the points in $[-T, T] \backslash\left\{s_{0}\right\}$ with

$$
u\left(s_{0}, y_{0}\right)=u\left(s_{1}, y_{0}\right)=\cdots=u\left(s_{N}, y_{0}\right) .
$$

Choose $r>0$ so small that the map $\left[s_{j}-r, s_{j}+r\right] \rightarrow X: s \mapsto u\left(s, y_{0}\right)$ is an embedding for every $j$. Shrinking $U$ if necessary, we may assume that this continues to hold for every $y \in U$.

Next we claim that there is a $\delta>0$ and a compact neighborhood $V \subset U$ of $y_{0}$ such that

$$
y \in V \quad \Longrightarrow \quad u\left(\left[s_{0}-\delta, s_{0}+\delta\right], y\right) \subset \bigcup_{j=1}^{N} u\left(\left[s_{j}-r, s_{j}+r\right], y\right) .
$$

If this were not the case, we could find sequences $\left(s_{\nu}, y_{v}\right) \rightarrow\left(s_{0}, y_{0}\right)$ and $s_{v}^{\prime} \in \mathbb{R} \backslash\left\{s_{v}\right\}$ such that $u\left(s_{v}, y_{v}\right)=u\left(s_{v}^{\prime}, y_{v}\right)$ and $\left|s_{v}^{\prime}-s_{j}\right|>r$ for all $j$ and $v$. By taking the limit $s_{v}^{\prime} \rightarrow s^{\prime}$ we would then obtain another element $s^{\prime} \notin\left\{s_{0}, \ldots, s_{N}\right\}$ with $u\left(s, y_{0}\right)=$ $u\left(s_{0}, y_{0}\right)$, a contradiction.

Now define the set

$$
\Sigma_{j}:=\left\{(s, y) \in\left[s_{0}-\delta, s_{0}+\delta\right] \times V \mid u(s, y) \in u\left(\left[s_{j}-r, s_{j}+r\right], y\right)\right\}
$$

for $j=1, \ldots, N$. These sets are closed and their union is the entire set $\left[s_{0}-\delta, s_{0}+\delta\right] \times V$. Hence, by Baire's category theorem, at least one of the sets $\Sigma_{j}$ must have nonempty 
interior. Assume without loss of generality that $\Sigma_{1}$ has nonempty interior and that $\left(s_{0}, y_{0}\right) \in \operatorname{int}\left(\Sigma_{1}\right)$. Choose a neighborhood $W \subset V$ of $y_{0}$ and a constant $\rho>0$ such that

$$
\left(s_{0}-\rho, s_{0}+\rho\right) \times W \subset \Sigma_{1} .
$$

Then for every pair $(s, y) \in\left(s_{0}-\rho, s_{0}+\rho\right) \times W$ there is a unique element $s^{\prime}=$ : $\sigma(s, y) \in\left[s_{1}-r, s_{1}+r\right]$ such that

$$
u(s, y)=u(\sigma(s, y), y) .
$$

This map $\sigma$ is evidently $C^{\ell-1}$ and satisfies $\sigma\left(s_{0}, y_{0}\right)=s_{1}$. Moreover,

$$
\begin{aligned}
0= & \partial_{s} u(s, y)-\nabla H(u(s, y))+I \partial_{v_{1}} u(s, y)+J \partial_{v_{2}} u(s, y)+K \partial_{v_{3}} u(s, y) \\
= & \left(\partial_{s} \sigma\right) \partial_{s} u(\sigma, y)-\nabla H(u(\sigma, y))+I\left(\partial_{v_{1}} u(\sigma, y)+\left(\partial_{v_{1}} \sigma\right) \partial_{s} u(\sigma, y)\right) \\
& +J\left(\partial_{v_{2}} u(\sigma, y)+\left(\partial_{v_{2}} \sigma\right) \partial_{s} u(\sigma, y)\right)+K\left(\partial_{v_{3}} u(\sigma, y)+\left(\partial_{v_{3}} \sigma\right) \partial_{s} u(\sigma, y)\right) \\
= & \left(\left(\partial_{s} \sigma-1\right) \mathbb{1}+\partial_{v_{1}} \sigma I+\partial_{v_{2}} \sigma J+\partial_{v_{3}} \sigma K\right) \partial_{s} u(\sigma, y) .
\end{aligned}
$$

Since $\partial_{s} u \neq 0$ the four vectors $\partial_{s} u, I \partial_{s} u, J \partial_{s} u, K \partial_{s} u$ are linearly independent and thus we obtain $\partial_{v_{i}} \sigma \equiv 0$ for $i=1,2,3$ and $\partial_{s} \sigma \equiv 1$. This means that

$$
\sigma(s, y)=s+s_{1}-s_{0}
$$

In other words, the solution $(s, y) \mapsto u\left(s+s_{1}-s_{0}, y\right)$ of (22) agrees with $u$ on an open set. By Proposition 4.4, this implies $u(s, y)=u\left(s+s_{1}-s_{0}, y\right)$ for all $s$ and $y$. Hence $f^{+}=f^{-}$, a contradiction. This proves the proposition.

Proof of Theorem 4.3 Fix a constant $p>4$. There is a Banach manifold $\mathcal{B}=$ $\mathcal{B}\left(f^{-}, f^{+}\right)$of all continuous maps $u: \mathbb{R} \times M \rightarrow X$ that are locally of class $W^{1, p}$ and, near infinity, can be written as

$$
u(s, y)=\exp _{f}(y)\left(\xi^{ \pm}(s, y)\right)
$$

with $\xi^{+} \in W^{1, p}\left([T, \infty) \times M,\left(f^{+}\right)^{*} T X\right)$ and similarly for $\xi^{-}$. Fix an element $H_{0} \in \mathcal{H}^{\text {morse }}$. Following Floer [10] we choose a separable Banach space $\mathcal{H}_{0} \subset \mathcal{H}$ of smooth functions $h: X \times M \rightarrow \mathbb{R}$ satisfying the following axioms.

(I) If $f \in \mathcal{C}\left(H_{0}\right)$ and $h \in \mathcal{H}_{0}$ then $h$ vanishes to infinite order at the point $(f(y), y)$ for every $y \in M$.

(II) Let $(x, y) \in X \times M$ such that $y \neq f(x)$ for every $f \in \mathcal{C}\left(H_{0}\right)$. Let $A: T_{x} X \rightarrow \mathbb{R}$ be a linear map. Then there are smooth functions $h: X \rightarrow \mathbb{R}, \alpha_{x}: X \rightarrow[0,1]$, and $\beta_{x}: M \rightarrow[0,1]$ such that the following holds. 
(a) $h(x)=0$ and $d h(x)=A$. Moreover, $\alpha$ and $\beta$ are supported in the balls of radius 1 about $x$ and $y$, respectively, and $\alpha(x)=\beta(y)=1$.

(b) For $\delta, \varepsilon>0$ define $\alpha_{x}^{\delta}: X \rightarrow[0,1]$ and $\beta_{y}^{\varepsilon}: M \rightarrow[0,1]$ by

$$
\alpha_{x}^{\delta}\left(\exp _{x}(\xi)\right):=\alpha_{x}\left(\exp _{x}\left(\delta^{-1} \xi\right)\right), \quad \beta_{y}^{\varepsilon}\left(\exp _{y}(\eta)\right):=\beta_{y}\left(\exp _{y}\left(\varepsilon^{-1} \eta\right)\right)
$$

Then the function $h^{\delta, \varepsilon}: M \times X \rightarrow \mathbb{R}$ given by

$$
h^{\delta, \varepsilon}\left(x^{\prime}, y^{\prime}\right):=\alpha_{x}^{\delta}\left(x^{\prime}\right) \beta_{y}^{\varepsilon}\left(y^{\prime}\right) h\left(x^{\prime}\right)
$$

belongs to $\mathcal{H}_{0}$ for $\delta, \varepsilon$ positive and sufficiently small.

To define the space $\mathcal{H}_{0}$ we choose a smooth cutoff function $\rho:[0, \infty) \rightarrow[0,1]$ such that $\rho(r)=1$ for $r$ sufficiently small and $\rho(r)=0$ for $r \geq r_{0}$, where $r_{0}$ is smaller than the injectivity radii of $X$ and $M$. For $x \in X$ and $y \in M$ define

$$
\alpha_{x}\left(\exp _{x}(\xi)\right):=\rho(|\xi|), \quad \beta_{y}\left(\exp _{y}(\eta)\right):=\rho(|\eta|)
$$

Then define $\mathcal{H}_{0}$ to be the set of all smooth functions $h: X \times M \rightarrow \mathbb{R}$ that vanish to infinite order along the graph of any element $f \in \mathcal{C}\left(H_{0}\right)$ and such that

$$
\|h\|_{c}:=\sum_{\ell=0}^{\infty} c_{\ell}^{-1}\|h\|_{C^{\ell}}<\infty, \quad c_{\ell}:=2^{2^{\ell}}\left(\sup _{x}\left\|\alpha_{x}\right\|_{C^{\ell}}+\sup _{y}\left\|\beta_{y}\right\|_{C^{\ell}}\right) .
$$

This space satisfies (I) and (II).

Consider the universal moduli space

$$
\mathcal{M}_{0}\left(f^{-}, f^{+}\right):=\left\{\left(u, H_{0}+h\right) \in \mathcal{B} \times \mathcal{H} \mid h \in \mathcal{H}_{0}, u \in \mathcal{M}\left(f^{-}, f^{+} ; H\right)\right\} .
$$

This space is the zero set of a smooth section of the Banach space bundle

$$
\mathcal{E} \rightarrow \mathcal{B} \times\left(H_{0}+\mathcal{H}_{0}\right)
$$

with fibers $\mathcal{E}_{u, H}=L^{p}\left(\mathbb{R} \times M, u^{*} T X\right)$. The section is $(u, H) \mapsto \partial_{s} u+\partial_{H}(u)$ and the claim below asserts that it is transverse to the zero section. Hence $\mathcal{M}_{0}\left(f^{-}, f^{+}\right)$ is a smooth Banach manifold. Now the obvious projection

$$
\pi_{0}: \mathcal{M}_{0}\left(f^{-}, f^{+}\right) \rightarrow H_{0}+\mathcal{H}_{0}
$$

is a Fredholm map. Hence, by the Sard-Smale theorem, the set of regular values of $\pi_{0}$ is of the second category in the sense of Baire in $H_{0}+\mathcal{H}_{0}$. Thus the set $\mathcal{H}^{\text {reg }}$ is dense in $\mathcal{H}$. Now we may introduce sets $\mathcal{H}_{c}^{\text {reg }} \supset \mathcal{H}^{\text {reg }}$ for $c>0$, as in the proof of Theorem 4.1, where the requirement of transversality is restricted to a compact set of Floer trajectories. These sets are all open and, by what we have just proved, they are also dense in $\mathcal{H}$. It then follows that $\mathcal{H}^{\text {reg }}$ is the intersection of countably many 
open and dense sets $\mathcal{H}_{c}^{\text {reg }}$ for $c=1,2,3, \ldots$ and hence is of the second category in the sense of Baire.

\section{Claim The operator}

$$
W^{1, p}\left(\mathbb{R} \times M, u^{*} T X\right) \times \mathcal{H}_{0} \rightarrow L^{p}\left(\mathbb{R} \times M, u^{*} T X\right),(\xi, h) \mapsto \mathcal{D}_{u, H}-\nabla h(u)
$$

is surjective for every $H \in \mathcal{H}$ and every $u \in \mathcal{M}\left(f^{-}, f^{+} ; H\right)$.

Let $1 / p+1 / q=1$ and suppose $\eta \in L^{q}\left(\mathbb{R} \times M, u^{*} T M\right)$ annihilates the image of the operator in the sense that

$$
\int_{-\infty}^{\infty} \int_{M}\left\langle\eta, \mathcal{D}_{u, H} \xi-\nabla h(u)\right\rangle \kappa \operatorname{dvol}_{M} d s=0
$$

for all $h \in \mathcal{H}_{0}$ and $\xi \in W^{1, p}\left(\mathbb{R} \times M, u^{*} T X\right)$. Then $\eta$ is smooth and

$$
\mathcal{D}_{u, H}^{*} \eta=0, \quad \int_{\mathbb{R} \times M}\langle\eta, \nabla h(u)\rangle \kappa \operatorname{dvol}_{M} d s=0
$$

for all $h \in \mathcal{H}_{0}$. We prove in three steps that $\eta$ vanishes identically.

Step 1 For every $s \in \mathbb{R}$ we have $\int_{M}\left\langle\eta, \partial_{s} u\right\rangle \kappa \operatorname{dvol}_{M}=0$.

Since $\mathcal{D}_{u, H} \partial_{s} u=0$ and $\mathcal{D}_{u, H}^{*} \eta=0$ we have

$$
\begin{aligned}
\frac{d}{d s} \int_{M}\left\langle\eta, \partial_{s} u\right\rangle \kappa \mathrm{dvol}_{M} & =\int_{M}\left(\left\langle\eta, \nabla_{s} \partial_{s} u\right\rangle+\int_{M}\left\langle\nabla_{s} \eta, \partial_{s} u\right\rangle\right) \kappa \operatorname{dvol}_{M} \\
& =\int_{M}\left(\left\langle\eta, \mathcal{D}_{u, H} \partial_{s} u\right\rangle-\int_{M}\left\langle\mathcal{D}_{u, H}^{*} \eta, \partial_{s} u\right\rangle\right) \kappa \operatorname{dvol}_{M} \\
& =0 .
\end{aligned}
$$

Here we have used the formulas $\mathcal{D}_{u, H}=\nabla_{s}+\mathcal{D}_{u, H}, \mathcal{D}_{u, H}^{*}=-\nabla_{s}+\mathcal{D}_{u, H}$, and the fact that $\mathbb{D}_{u, H}$ is self-adjoint. Since $\eta \in L^{q}$ and $\partial_{s} u \in L^{p}$, their inner product over $\mathbb{R} \times M$ is finite and this proves Step 1 .

Step $2 \eta(s, y)$ and $\partial_{s} u(s, y)$ are linearly dependent for all $(s, y) \in \mathbb{R} \times M$.

Suppose otherwise that $\partial_{s} u\left(s_{0}, y_{0}\right)$ and $\eta\left(s_{0}, y_{0}\right)$ are linearly independent for some $\left(s_{0}, y_{0}\right) \in \mathbb{R} \times M$. By Proposition 4.6 we may assume $\left(s_{0}, y_{0}\right) \in \mathcal{R}(u)$. Choose a compact interval $I \subset \mathbb{R}$ containing $s_{0}$ in its interior such that $I \times\left\{y_{0}\right\} \subset \mathcal{R}(u)$ and $I \rightarrow X: s \mapsto u\left(s, y_{0}\right)$ is an embedding. Then there are open neighborhoods $U \subset X$ of $u\left(s_{0}, y_{0}\right)$ and $V \subset M$ of $y_{0}$ such that

(*) if $y \in V$ and $s \in \mathbb{R}$ such that $u(s, y) \in U$ then $s \in I$. 
Otherwise there are sequences $s_{v} \in \mathbb{R} \backslash I$ and $y_{v} \rightarrow y_{0}$ such that $u\left(s_{v}, y_{v}\right)$ converges to $u\left(s_{0}, y_{0}\right)$. If $s_{v}$ is unbounded then $u\left(s_{0}, y_{0}\right) \in\left\{f^{-}\left(y_{0}\right), f^{+}\left(y_{0}\right)\right\}$, which is impossible because $\left(s_{0}, y_{0}\right) \in \mathcal{R}(u)$. Thus the sequence $s_{v}$ is bounded and hence has a limit point $s \in \mathbb{R} \backslash \operatorname{int}(I)$ with $u\left(s, y_{0}\right)=u\left(s_{0}, y_{0}\right)$. Since $s \neq s_{0}$ and $\left(s_{0}, y_{0}\right) \in \mathcal{R}(u)$ this is a contradiction.

Since $\partial_{s} u\left(s_{0}, y_{0}\right)$ and $\eta\left(s_{0}, y_{0}\right)$ are linearly independent, hypothesis (II) on the space $\mathcal{H}_{0}$ asserts that there is a smooth function $h_{0}: X \rightarrow \mathbb{R}$ and smooth cutoff functions $\alpha: X \rightarrow[0,1]$ and $\beta: M \rightarrow[0,1]$, centered at $x_{0}:=u\left(s_{0}, y_{0}\right)$ and $y_{0}$, respectively, such that

$$
h_{0}\left(u\left(s_{0}, y_{0}\right)\right)=0,\left.\frac{\partial}{\partial s}\right|_{s=s_{0}} h_{0}\left(u\left(s, y_{0}\right)\right)=0, d h_{0}\left(u\left(s_{0}, y_{0}\right)\right) \eta\left(s_{0}, y_{0}\right)=1,
$$

and such that the function $h^{\delta, \varepsilon}$ defined by

$$
h^{\delta}(x, y):=\alpha^{\delta}(x) h_{0}(x), \quad h^{\delta, \varepsilon}(x, y):=\beta^{\varepsilon}(y) h^{\delta}(x, y),
$$

is an element of $\mathcal{H}_{0}$ for $\delta, \varepsilon$ sufficiently small. If $\delta, \varepsilon$ are so small that $B_{\delta}\left(u\left(s_{0}, y_{0}\right)\right) \subset$ $U$ and $B_{\varepsilon}\left(y_{0}\right) \subset V$ then the function $(s, y) \mapsto h^{\delta, \varepsilon}(u(s, y), y)$ is supported in $I \times V$. Namely, if $h^{\delta, \varepsilon}(u(s, y), y) \neq 0$ then $u(s, y) \in U$ and $y \in V$ and hence $s \in I$, by (*).

Next we prove that

$$
\int_{\mathbb{R}} d h_{y_{0}}^{\delta}\left(u\left(s, y_{0}\right)\right) \eta\left(s, y_{0}\right) d s>0
$$

for $\delta>0$ sufficiently small. To see this we observe that there is a constant $c>0$, independent of $\delta$, such that the following holds. First,

$$
\left|s-s_{0}\right| \leq \frac{\delta}{c} \quad \Longrightarrow \quad \alpha^{\delta}\left(u\left(s, y_{0}\right)\right) d h_{y_{0}}\left(u\left(s, y_{0}\right)\right) \eta\left(s, y_{0}\right) \geq \frac{1}{2},
$$

because

$$
\alpha\left(u\left(s_{0}, y_{0}\right)\right)=d h_{y_{0}}\left(u\left(s_{0}, y_{0}\right)\right) \eta\left(s_{0}, y_{0}\right)=1
$$

and so the condition $\left|s-s_{0}\right| \leq \delta / c$ with $c$ sufficiently large guarantees $\alpha^{\delta}\left(u\left(s, y_{0}\right)\right) \geq$ $3 / 4$ and $d h_{y_{0}}\left(u\left(s, y_{0}\right)\right) \eta\left(s, y_{0}\right) \geq 2 / 3$. Second,

$$
\left|h_{y_{0}}\left(u\left(s, y_{0}\right)\right) d \alpha^{\delta}\left(u\left(s, y_{0}\right)\right) \eta\left(s, y_{0}\right)\right| \leq \frac{c\left|s-s_{0}\right|^{2}}{\delta} \leq c^{3} \delta,
$$

because the function $s \mapsto h_{y_{0}}\left(u\left(s, y_{0}\right)\right)$ vanishes to first order at $s=s_{0}$ and the first derivative of $\alpha^{\delta}$ is bounded by a constant times $1 / \delta$. The last inequality follows from the fact that $d \alpha^{\delta}\left(u\left(s, y_{0}\right)\right)=0$ for $\left|s-s_{0}\right| \geq c \delta$. Both estimates taken together show 
that

$$
\int_{\mathbb{R}} d h_{y_{0}}^{\delta}\left(u\left(s, y_{0}\right)\right) \eta\left(s, y_{0}\right) d s \geq \int_{s_{0}-c \delta}^{s_{0}+c \delta}\left(\frac{1}{2}-c^{3} \delta\right) d s=2 c \delta\left(\frac{1}{2}-c^{3} \delta\right) .
$$

Thus (52) holds for $\delta<1 / 2 c^{3}$.

Now choose $\varepsilon$ so small that

$$
\int_{\mathbb{R}} d h_{y}^{\delta}(u(s, y)) \eta(s, y) d s>0
$$

for every $y \in M$ with $d\left(y_{0}, y\right)<\varepsilon$. Then the integral in (51) is positive for the function $h(x, y)=h^{\delta, \varepsilon}(x, y)=\beta^{\varepsilon}(y) h^{\delta}(x, y)$. This proves Step 2 .

Step $3 \eta$ vanishes identically.

Assume, by contradiction, that $\eta \not \equiv 0$. Then, by Proposition 4.5, the set

$$
\mathcal{U}:=\left\{(s, y) \in \mathbb{R} \times M \mid \partial_{s} u(s, y) \neq 0, \eta(s, y) \neq 0\right\}
$$

is nonempty, open, and connected. By Step 2 , there is a continuous function $\lambda: \mathcal{U} \rightarrow$ $\mathbb{R} \backslash\{0\}$ such that $\eta(s, y)=\lambda(s, y) \partial_{s} u(s, y)$ for all $(s, y) \in \mathcal{U}$. Since $\mathcal{U}$ is connected, by Proposition 4.5, the function $\lambda$ cannot change sign. Suppose $\lambda>0$ on $\mathcal{U}$. (Otherwise replace $\eta$ by $-\eta$.) Then

$$
\left\langle\eta, \partial_{s} u\right\rangle=\lambda\left|\partial_{s} u\right|^{2}>0
$$

on $\mathcal{U}$ and $\left\langle\eta, \partial_{s} u\right\rangle=0$ on $\mathbb{R} \times M \backslash \mathcal{U}$. This contradicts Step 1 and proves Step 3, the claim, and the first assertion of the theorem. The second assertion follows from Proposition 4.2 and the infinite dimensional implicit function theorem.

The above proof follows essentially the argument in [12, Theorem 5.1]. There are, however, a few subtle but important differences. In the present setting we cannot remove the Hamiltonian term $\nabla H$ from the equation by a change of coordinates. Second, in symplectic Floer theory the complement $\mathcal{Z}:=(\mathbb{R} \times M) \backslash \mathcal{U}$ of the set $\mathcal{U}$ in Step 3 is discrete. This is replaced in the present context by the codimension 2 property of Proposition 4.5. In [12] the proof argues that $\partial_{s} \lambda \equiv 0$ and, because $\mathcal{Z}$ is discrete, that $\lambda$ can therefore be defined globally on $\mathbb{R} \times M$ (and not just on $\mathcal{U}$ ). The condition $\partial_{s} \lambda \equiv 0$ can also be obtained in the present case by the same argument, but we do not need it to obtain the contradiction.

The idea for the proof of the codimension 2 result was communicated to the third author, several years ago, by Kim Froyshov (in the context of Seiberg-Witten theory). This requires smooth perturbations and therefore we cannot work with the $C^{\ell}$ argument 
as in the proof of Theorem 4.1 but must instead use Floer's Banach spaces of smooth functions. As a result the construction of the function $h$ in Step 2 above is somewhat less explicit than in the proof of [12, Theorem 5.1].

\section{Floer homology}

We assume throughout that $M$ is a compact Cartan hypercontact 3-manifold and $X$ is a compact flat hyperkähler manifold. For $H \in \mathcal{H}^{\text {reg }}$ we introduce the chain complex

$$
\mathrm{CF}_{k}(M, X ; H):=\bigoplus_{\substack{f \in \mathcal{C}(H) \\ \mu_{H}(f)=k}} \mathbb{Z}_{2}\langle f\rangle .
$$

This group is finitely generated by Theorem 3.6. It is graded by the index function in Equation (50)

$$
\mu_{H}: \mathcal{C}(H) \rightarrow \mathbb{Z} .
$$

Since $H \in \mathcal{H}^{\text {reg }}$, Theorem 4.3 asserts that the moduli space $\mathcal{M}\left(f^{-}, f^{+} ; H\right)$ is a smooth manifolds of dimension $\mu_{H}\left(f^{-}\right)-\mu_{H}\left(f^{+}\right)$for every pair $f^{ \pm} \in \mathcal{C}(H)$. The real numbers act on these spaces by time shift and it follows from Theorem 3.15 that

$$
\mu_{H}\left(f^{-}\right)-\mu_{H}\left(f^{+}\right)=1 \quad \Longrightarrow \quad \# \mathcal{M}\left(f^{-}, f^{+} ; H\right) / \mathbb{R}<\infty .
$$

Thus we can use the numbers

$$
n_{2}\left(f^{-}, f^{+}\right):=\# \mathcal{M}\left(f^{-}, f^{+} ; H\right) / \mathbb{R} \text { (modulo 2) }
$$

to define a boundary operator $\partial^{H}: \mathrm{CF}_{k}(M, X ; H) \rightarrow \mathrm{CF}_{k-1}(M, X ; H)$ by

$$
\partial^{H}\left\langle f^{-}\right\rangle:=\sum_{\substack{f \in \mathcal{C}(H) \\ \mu_{H}(f+)=k-1}} n_{2}\left(f^{-}, f^{+}\right)\left\langle f^{+}\right\rangle
$$

for $f^{-} \in \mathcal{C}(H)$ with $\mu_{H}\left(f^{-}\right)=k$.

Theorem 5.1 For every $H \in \mathcal{H}^{\text {reg }}$ we have $\partial^{H} \circ \partial^{H}=0$.

To prove this one just needs to observe that the standard Floer gluing argument [6; $22 ; 27]$ carries over verbatim to the present setting. The Floer homology groups of $(M, X ; H)$ are now defined by

$$
\mathrm{HF}_{k}(M, X ; H):=\frac{\operatorname{ker} \partial^{H}: \mathrm{CF}_{k}(M, X ; H) \rightarrow \mathrm{CF}_{k-1}(M, X ; H)}{\operatorname{im} \partial^{H}: \mathrm{CF}_{k+1}(M, X ; H) \rightarrow \mathrm{CF}_{k}(M, X ; H)} .
$$

It follows again from the familiar arguments in symplectic Floer theory that these Floer homology groups are independent of the choice of the Hamiltonian perturbation 
$H \in \mathcal{H}^{\text {reg }}$. Here one can follow verbatim the discussion in [11;28], using the solutions of (22) with $H$ depending on $s$, to prove the following theorem.

Theorem 5.2 There is a natural family of isomorphisms

$$
\Phi^{\beta \alpha}: \operatorname{HF}_{*}\left(M, X ; H^{\alpha}\right) \rightarrow \operatorname{HF}_{*}\left(M, X ; H^{\beta}\right),
$$

one for each pair $H^{\alpha}, H^{\beta} \in \mathcal{H}^{\text {reg }}$, such that

$$
\Phi^{\gamma \beta} \circ \Phi^{\beta \alpha}=\Phi^{\gamma \alpha}, \quad \Phi^{\alpha \alpha}=\mathrm{id} .
$$

Theorem 5.3 Let $X$ be a compact flat hyperkähler manifold. Then there is a natural family of isomorphisms

$$
\Phi^{\alpha}: H_{*}\left(X ; \mathbb{Z}_{2}\right) \rightarrow \mathrm{HF}_{*}\left(M, X ; H^{\alpha}\right),
$$

one for every $H^{\alpha} \in \mathcal{H}^{\text {reg }}$, such that

$$
\Phi^{\beta}=\Phi^{\beta \alpha} \circ \Phi^{\alpha}
$$

The proof of Theorem 5.3 is based on the following result which asserts that the Floer chain complex agrees with the Morse complex for a special class of perturbations.

Theorem 5.4 Let $M$ be a compact Cartan hypercontact 3-manifold and $X$ be a compact flat hyperkähler manifold. Let $H: X \rightarrow \mathbb{R}$ be a Morse function whose gradient flow is Morse-Smale. Then there is a constant $\varepsilon_{0}>0$ such that the following holds for $0<\varepsilon \leq \varepsilon_{0}$. If $x^{ \pm}$are critical points of $H$ with index difference $\operatorname{ind}_{H}\left(x^{+}\right)-$ $\operatorname{ind}_{H}\left(x^{-}\right) \leq 1$ and $u$ : $\mathbb{R} \times M \rightarrow X$ is a finite energy solution of the Floer equation

$$
\partial_{s} u+\varepsilon^{-1} \not \partial(u)=\nabla H(u), \quad \lim _{s \rightarrow \pm \infty} u(s, y)=x^{ \pm},
$$

then $\operatorname{ind}_{H}\left(x^{+}\right)-\operatorname{ind}_{H}\left(x^{-}\right)=1$, the function $u(s, y)$ is independent of $y \in M$, and the operator $\mathcal{D}_{u, \varepsilon}:=\nabla_{s}+\varepsilon^{-1} \mathcal{D}-\nabla \nabla H(u)$ is surjective.

Remark 5.5 Equation (53) is equivalent, via rescaling, to the equation

$$
\partial_{s} \tilde{u}+\not(\tilde{u})=\varepsilon \nabla H(\tilde{u}), \quad \lim _{s \rightarrow \pm \infty} \tilde{u}(s, y)=x^{ \pm},
$$

for the function $\tilde{u}(s, y):=u(\varepsilon s, y)$. Since the limit points $x^{ \pm}$are constant (as functions of $y$ ) the energy is

$$
\frac{1}{\varepsilon} \mathcal{E}_{\varepsilon H}(\widetilde{u})=\mathcal{E}_{H}(u)=\int_{-\infty}^{\infty} \int_{M}\left|\partial_{s} u\right|^{2} \kappa=\kappa \operatorname{Vol}(M)\left(H\left(x^{+}\right)-H\left(x^{-}\right)\right) .
$$


The solutions of (54), and hence also those of (53), determine the boundary operator on $\operatorname{CF}(M, X ; \varepsilon H)$. Moreover, $\mathcal{D}_{u, \varepsilon}$ is surjective if and only if the operator $\mathcal{D}_{\widetilde{u}, \varepsilon H}$ in Proposition 4.2 is surjective.

The proof of Theorem 5.4 needs some preparations.

Lemma 5.6 Let $M$ be a compact hypercontact 3-manifold and $X$ be a flat hyperkähler manifold. If $H: M \times X \rightarrow \mathbb{R}$ is any smooth function and $u: \mathbb{R} \times M \rightarrow X$ is a solution of (22) then

$$
\int_{s_{0}}^{s_{1}} \int_{M}\left(\left|\nabla_{s} \partial_{s} u\right|^{2}+\left|\not D \partial_{s} u\right|^{2}\right) \kappa \leq\left(C+\frac{4}{r^{2}}\right) \int_{s_{0}-r}^{s_{1}+r} \int_{M}\left|\partial_{s} u\right|^{2} \kappa
$$

for all $s_{0}<s_{1}$ and $r>0$, where

$$
C:=2\|H\|_{C^{3}}\left\|\partial_{s} u\right\|_{L^{\infty}}+2\|H\|_{C^{2}}^{2} .
$$

Proof For $s \in \mathbb{R}$ define

$$
\phi(s):=\frac{1}{2} \int_{M}\left|\partial_{s} u\right|^{2} \kappa, \quad \psi(s):=\frac{1}{2} \int_{M}\left(\left|\nabla_{s} \partial_{s} u\right|^{2}+\left|\mathcal{D} \partial_{s} u\right|^{2}\right) \kappa .
$$

Then

$$
\begin{aligned}
\phi^{\prime \prime}(s) & =\int_{M}\left|\nabla_{s} \partial_{s} u\right|^{2} \kappa+\int_{M}\left\langle\nabla_{s} \nabla_{s} \partial_{s} u, \partial_{s} u\right\rangle \kappa \\
& =\int_{M}\left|\nabla_{s} \partial_{s} u\right|^{2} \kappa+\int_{M}\left\langle\nabla_{s} \nabla_{s} \nabla H(u), \partial_{s} u\right\rangle \kappa-\int_{M}\left\langle\nabla_{s} \partial_{s} u, \mathcal{D} \partial_{s} u\right\rangle \kappa .
\end{aligned}
$$

Here we have used the fact that $\mathcal{D}$ commutes with $\nabla_{s}$, because $X$ is flat, and that $\mathscr{D}$ is self-adjoint with respect to the $L^{2}$ inner product with weight $\kappa$. Since $\nabla_{s} \partial_{s} u=$ $\nabla_{s} \nabla H(u)-\not D D \partial_{s} u$ we obtain

$$
\phi^{\prime \prime}(s)=2 \psi(s)+\int_{M}\left\langle\nabla_{s} \nabla_{s} \nabla H(u), \partial_{s} u\right\rangle \kappa-\int_{M}\left\langle\nabla_{s} \nabla H(u), \mathbb{D} \partial_{s} u\right\rangle \kappa .
$$

Using the two inequalities $\left|\nabla_{s} \nabla_{s} \nabla H(u)\right| \leq\|H\|_{C^{3}}\left|\partial_{s} u\right|^{2}+\|H\|_{C^{2}}\left|\nabla_{s} \partial_{s} u\right|$ and $\left|\nabla_{s} \nabla H(u)\right| \leq\|H\|_{C^{2}}\left|\partial_{s} u\right|$ we obtain

$$
\begin{aligned}
\phi^{\prime \prime}(s) & \geq 2 \psi(s)-\int_{M}\left|\nabla_{s} \nabla_{s} \nabla H(u)\right|\left|\partial_{s} u\right| \kappa-\int_{M}\left|\nabla_{s} \nabla H(u)\right|\left|\mathcal{D} \partial_{s} u\right| \kappa \\
& \geq 2 \psi(s)-\|H\|_{C^{3}} \int_{M}\left|\partial_{s} u\right|^{3} \kappa-\|H\|_{C^{2}} \int_{M}\left(\left|\nabla_{s} \partial_{s} u\right|+\left|\mathcal{D} \partial_{s} u\right|\right)\left|\partial_{s} u\right| \kappa \\
& \geq \psi(s)-\left(\|H\|_{C^{3}}\left\|\partial_{s} u\right\|_{L^{\infty}}+\|H\|_{C^{2}}^{2}\right) \int_{M}\left|\partial_{s} u\right|^{2} \kappa \\
& =\psi(s)-C \phi(s) .
\end{aligned}
$$


Now let $r, R>0$. Then, for $0 \leq s \leq r$, we have

$$
\begin{aligned}
\int_{s_{0}}^{s_{1}} \psi-C \int_{s_{0}-r}^{s_{1}+r} & \leq \int_{s_{0}-s}^{s_{1}+s}(\psi-C \phi) \leq \int_{s_{0}-s}^{s_{1}+s} \phi^{\prime \prime} \\
& =\phi^{\prime}\left(s_{1}+s\right)-\phi^{\prime}\left(s_{0}-s\right) \\
& =\frac{d}{d s}\left(\phi\left(s_{1}+s\right)+\phi\left(s_{0}-s\right)\right) .
\end{aligned}
$$

Integrating this inequality from 0 to $t$ we obtain

$$
\frac{r}{2}\left(\int_{s_{0}}^{s_{1}} \psi-C \int_{s_{0}-r}^{s_{1}+r} \phi\right) \leq \phi\left(s_{1}+t\right)+\phi\left(s_{0}-t\right)
$$

for $r / 2 \leq t \leq r$. Integrating this inequality again from $r / 2$ to $r$ gives

$$
\int_{s_{0}}^{s_{1}} \psi \leq\left(C+\frac{4}{r^{2}}\right) \int_{s_{0}-r}^{s_{1}+r} \phi .
$$

This proves the lemma.

Lemma 5.7 Let $M, X$, and $H$ be as in Theorem 5.4. Then there are positive constants $\varepsilon_{0}$ and $C$ such that every solution $u$ of (53) with $0<\varepsilon \leq \varepsilon_{0}$ satisfies

$$
\sup _{\mathbb{R} \times M}\left|\partial_{s} u\right| \leq C, \quad \sup _{\mathbb{R} \times M}\left|\partial_{v_{i}} u\right| \leq C \varepsilon
$$

for $i=1,2,3$.

Proof It is convenient to work with the solutions

$$
\tilde{u}(s, y)=u(\varepsilon s, y)
$$

of Equation (54). The function $s \mapsto \mathcal{A}_{\varepsilon H}(\widetilde{u}(s, \cdot))$ is nonincreasing along $\tilde{u}$ and converges to $-\varepsilon \kappa \operatorname{Vol}(M) H\left(x^{-}\right)$as $s \rightarrow-\infty$. Hence

$$
\mathcal{A}(\widetilde{u}(s, \cdot))=\mathcal{A}_{\varepsilon H}(\tilde{u}(s, \cdot))+\int_{M} \varepsilon H(\widetilde{u}(s, \cdot)) \kappa \leq \varepsilon \kappa \operatorname{Vol}(M)\|H\|,
$$

where

$$
\|H\|:=\max H-\min H .
$$

The energy of $u$ can be estimated by

$$
\mathcal{E}_{\varepsilon H}(\widetilde{u})=\int_{-\infty}^{\infty} \int_{M}\left|\partial_{s} \tilde{u}\right|^{2} \kappa \operatorname{dvol}_{M} \leq \varepsilon \kappa \operatorname{Vol}(M)\|H\| .
$$

By Equation (43) in Lemma 3.10, we have

$$
\frac{1}{2} \int_{M}|d \tilde{u}|^{2} \leq \mathcal{A}(\tilde{u}(s, \cdot))+\varepsilon^{2} \operatorname{Vol}(M) \sup _{\mathbb{R} \times M}|\nabla H|^{2}+\frac{3}{2} \int_{M}\left|\partial_{s} \tilde{u}\right|^{2}
$$


for every $s \in \mathbb{R}$. Integrating this inequality from $s_{0}-1$ to $s_{0}+1$, and using (56) and (57) we obtain

$$
\int_{s_{0}-1}^{s_{0}+1} \int_{M}|d \tilde{u}|^{2} \leq c \varepsilon, \quad c:=(3+4 \kappa) \operatorname{Vol}(M)\|H\|+4 \operatorname{Vol}(M) \sup _{\mathbb{R} \times M}|\nabla H|^{2} .
$$

Hence, by Lemma 3.3 and Theorem B.1, there are positive constants $c^{\prime}$ and $\varepsilon_{0}$ such that $\sup |d \tilde{u}| \leq c^{\prime}$ for every solution of (54) with $0<\varepsilon \leq \varepsilon_{0}$.

To improve this estimate we observe that the constant in Lemma 5.6 with $H$ replaced by $\varepsilon H$ is

$$
C=2 \varepsilon^{2}\|H\|_{C^{2}}^{2}+2 \varepsilon\|H\|_{C^{3}}\left\|\partial_{s} \tilde{u}\right\|_{L^{\infty}} \leq c_{1} \varepsilon,
$$

where $c_{1}$ depends only on $H$ and the bound on $|d \tilde{u}|$ established above. Hence it follows from Lemma 5.6 with $r=\infty$ that

$$
\int_{-\infty}^{\infty} \int_{M}\left(\left|\nabla_{s} \partial_{s} \tilde{u}\right|^{2}+\left|D D \partial_{s} \tilde{u}\right|^{2}\right) \kappa \leq c_{1} \varepsilon \mathcal{E}_{\varepsilon H}(\widetilde{u}) \leq c_{2} \varepsilon^{2}
$$

Here we have used the fact that the energy of $d \tilde{u}$ is bounded by a constant times $\varepsilon$. Since $\int_{M} \partial_{s} \tilde{u}=\varepsilon \int_{M} \nabla H(u)$ we obtain from (59) with $\xi=\partial_{s} \tilde{u}$ that

$$
\int_{M}\left|\partial_{s} \tilde{u}\right|^{2} \leq c_{0}\left(\int_{M}\left|\not D \partial_{s} \tilde{u}\right|^{2}+\|H\|_{C^{1}}^{2} \varepsilon^{2}\right) .
$$

Integrating this inequality from $s_{0}-1$ to $s_{0}+1$ gives

$$
\int_{s_{0}-1}^{s_{0}+1} \int_{M}\left|\partial_{s} \tilde{u}\right|^{2} \leq c_{0} \int_{-\infty}^{\infty} \int_{M}\left|\not D \partial \partial_{s} \tilde{u}\right|^{2}+2 c_{0}\|H\|_{C^{1}}^{2} \varepsilon^{2} \leq c_{3} \varepsilon^{2} .
$$

Now it follows from Lemma 3.5 and Theorem B.1 that every solution of (54) with $0<\varepsilon \leq \varepsilon_{0}$ satisfies the pointwise inequality $\left|\partial_{s} \tilde{u}\right| \leq c_{4} \varepsilon$ for a suitable constant $c_{4}>0$. Using the equation we obtain $|\partial(\tilde{u})| \leq c_{5} \varepsilon$. Using again the fact that $\partial=\not D$ (on functions with values in $\mathbb{H}^{n}$ ) is an elliptic operator whose kernel consists of the constant functions we obtain $\int_{M}|d \tilde{u}|^{2} \leq c_{6} \varepsilon^{2}$ for every $s$. Integrating this inequality from $s_{0}-1$ to $s_{0}+1$, and using Lemma 3.3 and Theorem B.1, we conclude that every solution of (54) with $0<\varepsilon \leq \varepsilon_{0}$ satisfies the pointwise inequality $|d \tilde{u}|^{2} \leq c_{7} \varepsilon^{2}$ for a suitable constant $c_{7}>0$. This proves the lemma.

Lemma 5.8 Let $M, X$, and $H$ be as in Theorem 5.4. Then there are positive constants $\varepsilon_{0}, \delta$, and $c$ such that the following holds. If $f: M \rightarrow X$ is a smooth function such that

$$
\sup _{M}\left|\varepsilon^{-1} \partial(f)-\nabla H(f)\right|<\delta
$$


then

$$
\int_{M}|\xi|^{2} \leq c \int_{M}\left|\varepsilon^{-1} \mathcal{D} \xi-\nabla_{\xi} \nabla H(f)\right|^{2}
$$

for every $\xi \in \Omega^{0}\left(M, f^{*} T X\right)$.

Proof Suppose, by contradiction, that there are sequences $\varepsilon_{v} \rightarrow 0$ and $f_{v}: M \rightarrow X$ such that the sequence

$$
\eta_{v}:=\varepsilon_{v}^{-1} \partial\left(f_{v}\right)-\nabla H\left(f_{v}\right)
$$

converges uniformly to zero and (58) does not hold for $f_{v}$. It is convenient to choose lifts of the maps with values in the universal cover $\mathbb{H}^{n}$ of $X$. These lifts will still be denoted by $f_{v}: M \rightarrow \mathbb{H}^{n}$ and we introduce the sequence of mean values

$$
\overline{f_{v}}:=\frac{1}{\operatorname{Vol}(M)} \int_{M} f_{v}
$$

Assume without loss of generality that the sequence $\bar{f}_{v} \in \mathbb{H}^{n}$ is bounded and hence, passing to a subsequence if necessary, that it converges. By elliptic regularity for the operator $\mathbb{D}$ whose kernel consists of the constant functions (Lemma 3.7), there is a constant $c_{0}>0$ such that

$$
\int_{M} \xi=0 \Longrightarrow \int_{M}|\xi|^{2} \leq c_{0} \int_{M}|\mathbb{D} \xi|^{2}, \quad \sup _{M}|\xi| \leq c_{0} \sup _{M}|\mathbb{D} \xi|
$$

for every smooth map $\xi: M \rightarrow \mathbb{H}^{n}$. To prove the second inequality in (59) one can use the Sobolev estimate $\|\xi\|_{L^{\infty}} \leq c\|\xi\|_{W^{1, p}}$ for $\underline{p}>3$ and then $L^{p}$ regularity for $\mathbb{D}$. Applying this inequality to the sequence $f_{v}-\overline{f_{v}}$ we obtain

$$
\sup _{M}\left|f_{v}-\overline{f_{v}}\right| \leq c_{0} \sup _{M}\left|\partial\left(f_{v}\right)\right|=c_{0} \varepsilon_{v} \sup _{M}\left|\nabla H\left(f_{v}\right)+\eta_{v}\right| \rightarrow 0
$$

and so $f_{v}$ converges uniformly to the same limit as $\overline{f_{v}}$. Since

$$
\lim _{\nu \rightarrow \infty} \nabla H\left(\overline{f_{v}}\right)=\lim _{\nu \rightarrow \infty} \frac{1}{\operatorname{Vol}(M)} \int_{M} \nabla H\left(f_{\nu}\right)=\lim _{\nu \rightarrow \infty} \frac{1}{\operatorname{Vol}(M)} \int_{M} \eta_{\nu}=0
$$

this limit is a critical point of $H$. Hence there is a constant $c_{1}>0$ such that, for $v$ sufficiently large and $\bar{\xi} \in \mathbb{H}^{n}$, we have

$$
|\bar{\xi}| \leq c_{1}\left|\nabla_{\xi} \nabla H\left(\overline{f_{v}}\right)\right| .
$$

Now let $\xi: M \rightarrow \mathbb{H}^{n}$ be a smooth map (thought of as a vector field along $f_{v}$ ) and denote

$$
\bar{\xi}:=\frac{1}{\operatorname{Vol}(M)} \int_{M} \xi
$$


Then $\not{D} \xi$ has mean value zero and hence is $L^{2}$ orthogonal to $\nabla_{\xi} \nabla H\left(\overline{f_{v}}\right)$. This implies

$$
\begin{aligned}
& \varepsilon_{v}^{-2}\|\mathcal{D} \xi\|^{2}+\left|\nabla_{\xi} \nabla H\left(\overline{f_{v}}\right)\right|^{2}=\left\|\varepsilon_{v}^{-1} \mathbb{D} \xi-\nabla_{\xi} \nabla H\left(\overline{f_{v}}\right)\right\|^{2} \\
& \leq 3\left\|\varepsilon_{v}^{-1} \not{D} \xi-\nabla_{\xi} \nabla H\left(f_{v}\right)\right\|^{2}+3\left\|\nabla_{\xi-\bar{\xi}} \nabla H\left(f_{v}\right)\right\|^{2} \\
& \quad+3\left\|\nabla_{\bar{\xi}} \nabla H\left(f_{v}\right)-\nabla_{\bar{\xi}} \nabla H\left(\overline{f_{v}}\right)\right\|^{2} \\
& \leq 3\left\|\varepsilon_{v}^{-1} \mathbb{D} \xi-\nabla_{\xi} \nabla H\left(f_{v}\right)\right\|^{2}+c\|\xi-\bar{\xi}\|^{2}+c|\bar{\xi}|^{2}\left\|f_{v}-\overline{f_{v}}\right\|^{2} \\
& \leq 3\left\|\varepsilon_{v}^{-1} \mathbb{D} \xi-\nabla_{\xi} \nabla H\left(f_{v}\right)\right\|^{2}+c c_{0}\|\not D \xi\|^{2}+c c_{1}\left|\nabla_{\bar{\xi}} \nabla H\left(\overline{f_{v}}\right)\right|^{2}\left\|f_{v}-\bar{f}_{\mathcal{v}}\right\|^{2}
\end{aligned}
$$

Here all norms are $L^{2}$ norms on $M$, the constant $c$ depends only on $H$, and the last inequality follows from (59) and (60). For $v$ sufficiently large the last two terms one the right are together at most one quarter of the terms on the left. For these values of $v$ we have

$$
\varepsilon_{v}^{-2}\|\mathcal{D} \xi\|^{2}+\left\|\nabla_{\xi} \nabla H\left(\overline{f_{v}}\right)\right\|^{2} \leq 4\left\|\varepsilon_{v}^{-1} \not{D} \xi-\nabla_{\xi} \nabla H\left(f_{v}\right)\right\|^{2} .
$$

Hence if follows from (59) and (60) that $f_{v}$ satisfies (58) for $v$ sufficiently large, in contradiction to our assumption. This proves the lemma.

Lemma 5.9 Let $M, X$, and $H$ be as in Theorem 5.4. Then there are positive constants $\varepsilon_{0}, \delta, \rho$, and $c$ such that the following holds. If $T>0$ and $u: \mathbb{R} \times M \rightarrow X$ is a solution of (53) with $0<\varepsilon \leq \varepsilon_{0}$ such that

then

$$
\begin{gathered}
\int_{-T}^{T} \int_{M}\left|\partial_{s} u\right|^{2}<\delta \\
\sup _{y \in M}\left|\partial_{s} u(s, y)\right|^{2} \leq c e^{-\rho(T-|s|)} \int_{-T}^{T} \int_{M}\left|\partial_{S} u\right|^{2}
\end{gathered}
$$

for $|s| \leq T-2$.

Proof The functions

$$
\begin{aligned}
\phi(s) & :=\frac{1}{2} \int_{M}\left|\partial_{s} u\right|^{2}, \\
\psi(s) & :=\int_{M}\left|\nabla_{s} \partial_{s} u\right|^{2}+\int_{M}\left|\varepsilon^{-1} \mathcal{D} \partial_{s} u-\nabla_{\partial_{s}} \nabla H(u)\right|^{2}
\end{aligned}
$$

satisfy

$$
\begin{aligned}
\phi^{\prime \prime}(s) & =\psi(s)+\int_{M}\left\langle\nabla^{2} \nabla H\left(\partial_{s} u, \partial_{s} u\right), \partial_{s} u\right\rangle \\
& \geq \psi(s)-2\|H\|_{C^{3}}\left\|\partial_{s} u\right\|_{L^{\infty}(M)} \phi(s) .
\end{aligned}
$$


Hence, by Lemma 5.7, there is a constant $B>0$ such that $\phi^{\prime \prime} \geq-B \phi$. Now apply Theorem B.1 to the function $\phi$ to obtain

$$
|s| \leq T-1 \quad \Longrightarrow \quad \phi(s) \leq c_{1} \int_{s-1}^{s+1} \phi \leq c_{1} \int_{-T}^{T} \phi \leq c_{1} \delta .
$$

Careful inspection of the proof of Theorem B.1 for $n=1$ shows that the constant can be chosen as $c_{1}=8(\sqrt{B}+1)$. This shows that the rescaled function $\tilde{u}(s, y):=u(\varepsilon s, y)$ satisfies the inequality

$$
|s| \leq \varepsilon^{-1}(T-1) \quad \Longrightarrow \quad \int_{M}\left|\partial_{s} \tilde{u}\right|^{2} \leq c_{1} \varepsilon^{2} \delta .
$$

Now integrate this inequality from $s_{0}-1$ to $s_{0}+1$. Using Lemma 3.5 (together with the uniform $C^{1}$ bound of Lemma 5.7) and Theorem B.1 we then obtain the pointwise inequality $\left|\partial_{s} \tilde{u}(s, y)\right|^{2} \leq c_{2} \varepsilon^{2} \delta$ for $|s| \leq \varepsilon^{-1}(T-1)-1$. For the function $u$ this gives

$$
|s| \leq T-2 \Longrightarrow \sup _{M}\left|\varepsilon^{-1} \not D \partial_{s} u-\nabla H(u)\right|^{2}=\sup _{M}\left|\partial_{s} u\right|^{2} \leq c_{2} \delta .
$$

If $\delta$ is chosen sufficiently small we obtain from Lemma 5.8 with $\xi=\partial_{s} u$ that

$$
\int_{M}\left|\partial_{s} u\right|^{2} \leq c_{3} \int_{M}\left|\varepsilon^{-1} \not{D} \partial_{s} u-\nabla_{\partial_{s}} \nabla H(u)\right|^{2}
$$

for $|s| \leq T-2$. Thus $\phi(s) \leq c_{3} \psi(s)$ and, putting things together, we have

$$
\begin{aligned}
\phi^{\prime \prime}(s) & \geq \psi(s)-2\|H\|_{C^{3}}\left\|\partial_{s} u\right\|_{L^{\infty}(M)} \phi(s) \\
& \geq\left(\frac{1}{c_{3}}-2\|H\|_{C^{3}} \sqrt{c_{2} \delta}\right) \phi(s)
\end{aligned}
$$

for $|s| \leq T-2$. With $\delta$ sufficiently small this gives $\phi^{\prime \prime}(s) \geq \rho^{2} \phi(s)$ and hence the function $s \mapsto e^{-\rho s}\left(\phi^{\prime}(s)+\rho \phi(s)\right)$ is nondecreasing. If $\phi^{\prime}\left(s_{0}\right) \geq 0$ we then obtain $e^{-\rho s_{0}} \rho \phi\left(s_{0}\right) \leq e^{-\rho s_{0}}\left(\phi^{\prime}\left(s_{0}\right)+\rho \phi\left(s_{0}\right)\right) \leq e^{-\rho s}\left(\phi^{\prime}(s)+\rho \phi(s)\right)$ for $s_{0} \leq s \leq T-2$. Thus $\rho e^{\rho\left(s-s_{0}\right)} \phi\left(s_{0}\right) \leq \phi^{\prime}(s)+\rho \phi(s)$ and integrating this inequality gives

$$
e^{\rho\left(T-s_{0}-2\right)} \phi\left(s_{0}\right) \leq \phi(T-2)+\rho \int_{s_{0}}^{T-2} \phi \leq\left(c_{1}+\rho\right) \int_{-T}^{T} \phi .
$$

If $\phi^{\prime}\left(s_{0}\right) \leq 0$ we obtain a similar inequality by reversing time. Thus we have proved that $e^{\rho(T-|s|)} \phi(s) \leq c_{4} \int_{-T}^{T} \phi$ for $|s| \leq T-2$, where $c_{4}:=e^{2 \rho}\left(c_{1}+\rho\right)$. The pointwise estimate for $\left|\partial_{s} u\right|^{2}$ follows by the same argument as above from Lemma 3.5 and Theorem B.1 via rescaling. This proves the lemma.

Proof of Theorem 5.4 The proof has four steps. It is modelled on the adiabatic limit argument in [8]. 
Step 1 There exists a constant $\varepsilon_{0}>0$ with the following significance. If $0<\varepsilon \leq \varepsilon_{0}$, $x^{ \pm}$are critical points of $H$ with $\operatorname{ind}_{H}\left(x^{+}\right)-\operatorname{ind}_{H}\left(x^{-}\right)=1$, and $u_{0}: \mathbb{R} \rightarrow X$ is a gradient trajectory from $x^{-}$to $x^{+}$for $\varepsilon H$, ie

$$
\frac{d}{d s} u_{0}(s)=\nabla H\left(u_{0}(s)\right), \quad \lim _{s \rightarrow \pm \infty} u_{0}(s)=x^{ \pm},
$$

then the function $\mathbb{R} \times M \rightarrow X:(s, y) \mapsto u_{0}(s)$ is a regular solution of (53), ie the operator $\mathcal{D}_{u_{0}, \varepsilon}$ is surjective.

Let $\xi \in W^{1, p}\left(\mathbb{R} \times M, u_{0}^{*} T X\right)$ and define $\bar{\xi} \in W^{1, p}\left(\mathbb{R}, u_{0}^{*} T X\right)$ by

$$
\bar{\xi}(s):=\frac{1}{\operatorname{Vol}(M)} \int_{M} \xi(s, y) \operatorname{dvol}_{M}(y)
$$

for $s \in \mathbb{R}$. Then

$$
\mathcal{D}_{u_{0}, \varepsilon} \xi=\partial_{s} \bar{\xi}+\nabla_{\bar{\xi}} \nabla H\left(u_{0}\right)+\mathcal{D}_{u_{0}, \varepsilon}(\xi-\bar{\xi}) .
$$

Denote by $W_{0}^{1, p}\left(\mathbb{R} \times M, u_{0}^{*} T X\right) \subset W^{1, p}\left(\mathbb{R} \times M, u_{0}^{*} T X\right)$ the subspace of all functions $\xi$ such that $\xi(s, \cdot)$ has mean value zero on $M$ for every $s$ and similarly for $L_{0}^{p}\left(\mathbb{R} \times M, u_{0}^{*} T X\right) \subset L^{p}\left(\mathbb{R} \times M, u_{0}^{*} T X\right)$. Then the operator

$$
\mathcal{D}_{u_{0}, \varepsilon}: W_{0}^{1, p}\left(\mathbb{R} \times M, u_{0}^{*} T X\right) \rightarrow L_{0}^{p}\left(\mathbb{R} \times M, u_{0}^{*} T X\right)
$$

is equivalent to the operator

$$
\mathcal{D}_{\tilde{u}_{0}, \varepsilon H}=\nabla_{s}+\not D D-\varepsilon \nabla \nabla H\left(\tilde{u}_{0}\right)
$$

associated to the rescaled function $\tilde{u}_{0}(s):=u_{0}(\varepsilon s)$. This operator is bijective for $\varepsilon=0$ and hence also for $\varepsilon>0$ sufficiently small. Hence Step 1 follows from the above decomposition of the operator $\mathcal{D}_{u_{0}, \varepsilon}$ (and the fact that there are only finitely many index one gradient trajectories up to time shift).

Step 2 There is a constants $\varepsilon_{0}>0$ with the following significance. If $x^{ \pm}$are critical points of $H$ such that $\operatorname{ind}_{H}\left(x^{+}\right)-\operatorname{ind}_{H}\left(x^{-}\right)=1$, and $u$ : $\mathbb{R} \times M \rightarrow X$ and $u_{0}: \mathbb{R} \rightarrow X$ are solutions of (53) and (61), respectively, such that

$$
0<\varepsilon \leq \varepsilon_{0}, \quad \sup _{\mathbb{R} \times M} d\left(u, u_{0}\right)<\delta
$$

then there is an $s_{0} \in \mathbb{R}$ such that $u\left(s+s_{0}, y\right)=u_{0}(s)$ for all $s$ and $y$.

We wish to find a real number $s_{0}$ close to zero such that

$$
\frac{1}{\operatorname{Vol}(M)} \int_{M} u\left(s_{0}, \cdot\right)-u_{0}(0) \perp \nabla H\left(u_{0}(0)\right) \text {. }
$$


To prove that $s_{0}$ exists we consider the function

$$
\phi(s):=\frac{1}{\operatorname{Vol}(M)} \int_{M}\left\langle u(s, \cdot)-u_{0}(0), \nabla H\left(u_{0}(0)\right)\right\rangle \mathrm{dvol}_{M} .
$$

It satisfies

$$
|\phi(0)| \leq \delta \mu, \quad \mu:=\left|\nabla H\left(u_{0}(0)\right)\right|>0 .
$$

Choose a constant $\rho>0$ so small that

$$
\left|x-u_{0}(0)\right| \leq \rho \quad \Longrightarrow \quad\left\langle\nabla H(x), \nabla H\left(u_{0}(0)\right)\right\rangle>\frac{\mu^{2}}{2} .
$$

Let $C$ be the constant of Lemma 5.7 so that $\sup _{\mathbb{R} \times M}\left|\partial_{s} u\right| \leq C$. Then we have $\left|u(s, y)-u_{0}(0)\right| \leq|u(s, y)-u(0, y)|+\delta \leq C|s|+\delta$, and hence

$$
C|s|+\delta \leq \rho \quad \Longrightarrow \quad\left|u(s, y)-u_{0}(0)\right| \leq \rho .
$$

Combining the last two inequalities we have, for $C|s|+\delta<\rho$, that

$$
\begin{aligned}
\dot{\phi}(s) & =\frac{1}{\operatorname{Vol}(M)} \int_{M}\left\langle\partial_{s} u(s, \cdot), \nabla H\left(u_{0}(0)\right)\right\rangle \\
& =\frac{1}{\operatorname{Vol}(M)} \int_{M}\left\langle\nabla H(u(s, \cdot)), \nabla H\left(u_{0}(0)\right)\right\rangle \\
& \geq \frac{\mu^{2}}{2} .
\end{aligned}
$$

To obtain a zero of $\phi$ we need this inequality on an interval of length $T$ (on either side of zero) where $\frac{1}{2} \mu^{2} T \geq \delta \mu$, or equivalently $T \geq 2 \delta / \mu$. On the other hand, the interval at our disposal has length at most $(\rho-\delta) / C$. Thus we must impose the condition $(\rho-\delta) / C>2 \delta / \mu$, or equivalently

$$
\delta\left(1+\frac{2 C}{\mu}\right)<\rho
$$

Under this assumption there is a real number $s_{0}$ with $\left|s_{0}\right| \leq 2 \delta / \mu$ such that (62) holds. We can still control the distance of $u\left(s+s_{0}, y\right)$ and $u_{0}(s)$ by a fixed multiple of $\delta$. We assume from now on that $\left|u\left(s+s_{0}, y\right)-u_{0}(s)\right| \leq c \delta$ for all $s$ and $y$ and that (62) holds.

Consider the functions

$$
\begin{aligned}
& \xi(s, y):=u\left(s+s_{0}, y\right)-u_{0}(s), \\
& \eta(s, y):=\nabla H\left(u\left(s+s_{0}, y\right)\right)-\nabla H\left(u_{0}(s)\right)-\nabla_{\xi(s, y)} \nabla H\left(u_{0}(s)\right) .
\end{aligned}
$$


Then $|\eta(s, y)| \leq\|H\|_{C^{3}}|\xi(s, y)|^{2}$ and

$$
\partial_{s} \xi+\varepsilon^{-1} \mathcal{D} \xi-\nabla_{\xi} \nabla H\left(u_{0}\right)=\eta .
$$

Hence the functions

$$
\bar{\xi}(s):=\frac{1}{\operatorname{Vol}(M)} \int_{M} \xi(s, \cdot), \quad \bar{\eta}(s):=\frac{1}{\operatorname{Vol}(M)} \int_{M} \eta(s, \cdot)
$$

satisfy

$$
\partial_{s} \bar{\xi}-\nabla_{\bar{\xi}} \nabla H\left(u_{0}\right)=\bar{\eta}, \quad\left\langle\bar{\xi}(0), \nabla H\left(u_{0}(0)\right)\right\rangle=0 .
$$

Since the gradient flow of $H$ is Morse-Smale and $u_{0}$ is an index-1 gradient trajectory of $H$ the kernel of the operator

$$
D_{u_{0}}:=\partial_{s}+\nabla \nabla H\left(u_{0}\right): W^{1, p}\left(\mathbb{R}, \mathbb{H}^{n}\right) \rightarrow L^{p}\left(\mathbb{R}, \mathbb{H}^{n}\right)
$$

is 1 -dimensional and is spanned by $\partial_{s} \tilde{u}_{0}$. Since $\partial_{s} u_{0}(0)=\nabla H\left(u_{0}(0)\right)$ the restriction of $D_{u_{0}}$ to the codimension-1 subspace of all $\zeta \in W^{1, p}\left(\mathbb{R}, \mathbb{H}^{n}\right)$ that satisfy $\left\langle\zeta(0), \nabla H\left(u_{0}(0)\right)\right\rangle=0$ is a Banach space isomorphism. This implies that there is a constant $c_{0}>0$, depending only on $u_{0}$, such that

$$
\left\langle\zeta(0), \nabla H\left(u_{0}(0)\right)\right\rangle=0 \quad \Longrightarrow \quad\|\zeta\|_{W^{1, p}} \leq c_{0}\left\|\partial_{s} \zeta-\nabla_{\zeta} \nabla H\left(u_{0}\right)\right\|_{L^{p}} .
$$

Applying this to the elements $\zeta=\bar{\xi}$ we have $D_{u_{0}} \bar{\xi}=\bar{\eta}$ and hence

$$
\|\bar{\xi}\|_{L^{p}} \leq c_{0}\|\bar{\eta}\|_{L^{p}} \leq \frac{c_{0}}{\operatorname{Vol}(M)^{1 / p}}\|\eta\|_{L^{p}} \leq \frac{c_{0} c\|H\|_{C^{3}}}{\operatorname{Vol}(M)^{1 / p}} \delta \xi \|_{L^{p}}
$$

Here we have used the inequality $|\eta| \leq\|H\|_{C^{3}}|\xi|^{2} \leq c\|H\|_{C^{3}} \delta|\xi|$. Now it follows from (63) and the discussion in the proof of Step 1 for the rescaled operator $\mathcal{D}_{\widetilde{u}_{0}, \varepsilon H}$ that, for a suitable constant (still denoted by $c_{0}$ ) and $\varepsilon>0$ sufficiently small, we have

$$
\begin{aligned}
\|\xi-\bar{\xi}\|_{L^{p}} & \leq c_{0} \varepsilon\|\eta-\bar{\eta}\|_{L^{p}} \\
& \leq c_{0} \varepsilon\left(1+\frac{1}{\operatorname{Vol}(M)^{1 / p}}\right)\|\eta\|_{L^{p}} \\
& \leq \frac{c_{0} c\|H\|_{C^{3}}}{\operatorname{Vol}(M)^{1 / p}}\left(\varepsilon+\varepsilon \operatorname{Vol}(M)^{1 / p}\right) \delta\|\xi\|_{L^{p}} .
\end{aligned}
$$

If $\delta\left(1+\varepsilon+\varepsilon \operatorname{Vol}(M)^{1 / p}\right)<\operatorname{Vol}(M)^{1 / p} / c_{0} c\|H\|_{C^{3}}$ then $\xi$ must vanish and this proves Step 2 .

Step 3 There are positive constant $\varepsilon_{0}$ and $c$ such that the following holds. If $x^{ \pm}$are critical points of $H$ and $u: \mathbb{R} \times M \rightarrow \mathbb{H}^{n}$ is a lift of a solution of (53) (with $0<\varepsilon \leq \varepsilon_{0}$ ) 
to the universal cover $\mathbb{H}^{n}$ of $X$, then the function

$$
\bar{u}(s):=\frac{1}{\operatorname{Vol}(M)} \int_{M} u(s, \cdot) \mathrm{dvol}_{M}
$$

satisfies the inequalities

$$
\int_{-\infty}^{\infty}\left|\partial_{s} \bar{u}(s)\right|^{2} d s \leq H\left(x^{+}\right)-H\left(x^{-}\right)+c \varepsilon^{2}, \quad \int_{-\infty}^{\infty}\left|\nabla_{s} \partial_{s} \bar{u}(s)\right|^{2} d s \leq c,
$$

and $\left|\partial_{s} \bar{u}(s)-\nabla H(\bar{u}(s))\right| \leq c \varepsilon$ for every $s \in \mathbb{R}$.

First note that

$$
\partial_{s} \bar{u}(s)-\varepsilon \nabla H(\bar{u}(s))=\frac{1}{\operatorname{Vol}(M)} \int_{M}(\nabla H(u(s, \cdot))-\nabla H(\bar{u}(s))) \operatorname{dvol}_{M}
$$

and hence

$$
\begin{aligned}
\left|\partial_{s} \bar{u}(s)-\nabla H(\bar{u}(s))\right|^{2} & \leq \frac{\|H\|_{C^{2}}}{\operatorname{Vol}(M)} \int_{M}|u(s, \cdot)-\bar{u}(s)|^{2} \operatorname{dvol}_{M} \\
& \leq \frac{c_{1}\|H\|_{C^{2}} \varepsilon^{2}}{\operatorname{Vol}(M)} \int_{M}|\partial(u)|^{2} \operatorname{dvol}_{M} \\
& \leq c_{2} \varepsilon^{4} .
\end{aligned}
$$

Here the second inequality follows from (59) and the last from Lemma 5.7. Second, the function $\bar{u}$ satisfies

$$
\int_{-\infty}^{\infty}\left|\nabla_{s} \partial_{s} \bar{u}(s)\right|^{2} d s \leq \frac{1}{\operatorname{Vol}(M)} \int_{-\infty}^{\infty} \int_{M}\left|\nabla_{s} \partial_{s} u\right|^{2} \operatorname{dvol}_{M} d s \leq c_{3}
$$

Here we have used Lemma 5.7 and Lemma 5.6 for the rescaled function $\widetilde{u}(s, y):=$ $u(\varepsilon s, y)$ with $C$ equal to a constant times $\varepsilon^{2}$. Third, we have

$$
\begin{aligned}
\int_{-\infty}^{\infty}\left|\partial_{s} \bar{u}(s)\right|^{2} d s & =\frac{1}{\operatorname{Vol}(M)} \int_{-\infty}^{\infty} \int_{M}\left(\left|\partial_{s} u\right|^{2}-\left|\partial_{s} u-\partial_{s} \bar{u}\right|^{2}\right) \\
& \leq H\left(x^{+}\right)-H\left(x^{-}\right)+\frac{c_{0}}{\operatorname{Vol}(M)} \int_{-\infty}^{\infty} \int_{M}\left|\mathcal{D} \partial_{s} u\right|^{2} \\
& \leq H\left(x^{+}\right)-H\left(x^{-}\right)+c_{4} \varepsilon^{2} .
\end{aligned}
$$

Here we have used (59) and Lemma 5.6, again for the rescaled function $\widetilde{u}(s, y):=$ $u(\varepsilon s, y)$. This proves Step 3 .

Step 4 We prove the theorem. 
Let $x^{ \pm}$be a pair of critical points of $H$ of index difference less than or equal to 1 . Suppose, by contradiction, that there is a sequence of solutions $u_{v}: \mathbb{R} \times M \rightarrow X$ of (53) associated to a sequence $\varepsilon_{v} \rightarrow 0$ such that $u_{v}(s, y)$ is not independent of $y$. Replace each $u_{v}$ by a lift to the universal cover $\mathbb{H}^{n}$ of $X$ (still denoted by $u_{v}$ ) with the same limit point $\lim _{s \rightarrow-\infty} u_{v}(s, y)$.

First, by Lemma 5.9, the functions $s \mapsto \partial_{s} u_{v}(s, y)$ satisfy a uniform $L^{1}$ bound. Namely, if $\delta$ is the constant of Lemma 5.9 and $N>\operatorname{Vol}(M)\left(H\left(x^{+}\right)-H\left(x^{-}\right)\right) / \delta$ is an integer then, for each $v$, the real axis can be divided into $N$ intervals such that the energy of $u_{\nu}$ on each of these intervals is less than $\kappa \delta$ and hence, by Lemma 5.9, $\partial_{s} u_{v}$ satisfies uniform exponential estimates on all these intervals. This shows that the images of the functions $u_{v}$ are contained in a fixed compact subset of $\mathbb{H}^{n}$.

Now consider the associated functions

$$
\bar{u}_{v}(s):=\frac{1}{\operatorname{Vol}(M)} \int_{M} u(s, \cdot) \mathrm{dvol}_{M} .
$$

Normalize the sequence such that $H\left(\bar{u}_{v}(0)\right)=2^{-1}\left(H\left(x^{+}\right)+H\left(x^{-}\right)\right)$. The $W^{2,2}-$ bound of Step 3 guarantees the existence of a subsequence (still denoted by $\bar{u}_{v}$ ) that converges in the $C^{1}$-norm on every compact subset of $\mathbb{R}$ to a gradient trajectory $\bar{u}_{\infty}$ of $H$. The energy bound of Step 3 shows that the limit sequence has energy at most $H\left(x^{+}\right)-H\left(x^{-}\right)$. We claim that $\bar{u}_{\infty}$ connects $x^{-}$to $x^{+}$. Otherwise, the standard compactness argument would give a subsequence converging to a catenation of at least two gradient trajectories running from $x^{-}$to $x^{+}$, contradicting the Morse-Smale property of the gradient flow. Now it follows from Step 3 that

$$
\int_{-\infty}^{\infty}\left|\partial_{s} \bar{u}_{\infty}\right|^{2}=H\left(x^{+}\right)-H\left(x^{-}\right)=\lim _{\nu \rightarrow \infty} \int_{-\infty}^{\infty}\left|\partial_{s} \bar{u}_{\nu}\right|^{2} .
$$

This implies that $\bar{u}_{v}\left(s_{v}\right)$ must converge to $x^{ \pm}$for every sequence $s_{v} \rightarrow \pm \infty$. Hence $\bar{u}_{v}$ converges uniformly to $\bar{u}_{\infty}$ on all of $\mathbb{R}$. Now it follows from the Sobolev inequality and the elliptic estimate for the operator $\mathbb{D}$ that

$$
\left\|u_{v}(s, \cdot)-\bar{u}_{v}(s)\right\|_{L^{\infty}(M)} \leq c_{1}\left\|\not D u_{v}(s, \cdot)\right\|_{L^{p}(M)} \leq c_{2} \varepsilon_{v}
$$

for $p>3$. Here the last inequality follows from Lemma 5.7. Hence

$$
\lim _{\nu \rightarrow \infty} \sup _{s, y}\left|u_{\nu}(s, y)-\bar{u}_{\infty}\left(\varepsilon_{v} s\right)\right|=0 .
$$

By Step 2 this implies that, for $v$ sufficiently large, $u_{v}(s, y)$ agrees with $\bar{u}_{\infty}(s)$ up to a time shift and hence is independent of $y$. This contradicts our assumption and proves the theorem. 
Lemma 5.10 Let $M, X$, and $H$ be as in Theorem 5.4. Then there is a constant $\varepsilon_{0}>0$ such that every smooth solution $f: M \rightarrow X$ of the equation

$$
\not(f)=\varepsilon \nabla H(f)
$$

with $0<\varepsilon<\varepsilon_{0}$ is constant.

Proof Since $X$ is flat we may replace $X$ by its universal cover $\mathbb{H}^{n}$ and use the fact that $\partial(f)=\not D D$ in this setting. It follows from Lemmas 2.2 and 3.7 that there is a constant $c_{1}>0$ such that

$$
\|d f\|_{L^{2}} \leq c_{1}\|\mathcal{D} f\|_{L^{2}}
$$

for every smooth map $f: M \rightarrow \mathbb{H}^{n}$. Moreover, Equation (42) shows that the second order differential operator $\mathbb{D} \mathbb{D}: W^{2,2}\left(M, \mathbb{H}^{n}\right) \rightarrow L^{2}\left(M, \mathbb{H}^{n}\right)$ is Fredholm and has index zero. Its kernel agrees with the kernel of $\mathscr{D}$. Hence there is a constant $c_{2}>0$ such that

$$
\|\not D f\|_{L^{2}} \leq c_{2}\|\not D D D f\|_{L^{2}}
$$

for every smooth map $f: M \rightarrow \mathbb{H}^{n}$. If $\mathcal{D} f=\varepsilon \nabla H(f)$ we obtain

$$
\not D D D f=\varepsilon\left(I \nabla_{\partial_{v_{1}}} \nabla H(f)+J \nabla_{\partial_{v_{2}} f} \nabla H(f)+K \nabla_{\partial_{v_{3}} f} \nabla h(f)\right)
$$

and hence

$$
\|\not D D D f\|_{L^{2}} \leq \varepsilon\left\|\nabla^{2} H\right\|_{L^{\infty}}\|d f\|_{L^{2}} .
$$

This gives

$$
\|d f\|_{L^{2}} \leq \varepsilon c_{1} c_{2}\left\|\nabla^{2} H\right\|_{L^{\infty}}\|d f\|_{L^{2}}
$$

for every solution $f: M \rightarrow \mathbb{H}^{n}$ of (64). With $\varepsilon c_{1} c_{2}\left\|\nabla^{2} H\right\|_{L^{\infty}}<1$ this implies that every solution is constant as claimed.

Proof of Theorem 5.3 Let $H: X \rightarrow \mathbb{R}$ be as in Theorem 5.4. If $\varepsilon>0$ is sufficiently small then, by Lemma 5.10, all critical points of $\mathcal{A}_{\varepsilon H}$ are constant and, by Theorem 5.4, each Floer trajectory for $\varepsilon H$ of index 1 is a Morse gradient line and there are no nontrivial Floer trajectories with index less than 1 . Thus, for $H^{\prime} \in \mathcal{H}^{\text {reg }}$ sufficiently $C^{2}$ close to $\varepsilon H$, the Floer chain complex $\left(\mathrm{CF}\left(M, X ; H^{\prime}\right), \partial^{H^{\prime}}\right)$ coincides with the Morse complex of $\varepsilon H$. Hence the Floer homology group $\operatorname{HF}\left(M, X ; H^{\prime}\right)$ is naturally isomorphic to the Morse homology of $(X, \varepsilon H)$. This gives rise to an isomorphism $H_{*}\left(X ; \mathbb{Z}_{2}\right) \rightarrow \operatorname{HF}\left(M, X ; H^{\prime}\right)$ and composition with the isomorphisms $\operatorname{HF}\left(M, X ; H^{\prime}\right) \rightarrow \operatorname{HF}\left(M, X ; H^{\alpha}\right)$ of Theorem 5.2 gives a family of isomorphisms satisfying the requirements of Theorem 5.3.

Proof of Theorem 2.7 Assume $X$ is a compact flat hyperkähler manifold and let $H \in \mathcal{H}^{\text {morse }}$. Then, by Theorem 3.6, the number of critical points of $\mathcal{A}_{H}$ remains unchanged under any perturbation of $H$ that is sufficiently small in the $C^{2}$ norm. 
Hence, by Theorem 4.3, we may assume without loss of generality that $H \in \mathcal{H}^{\text {reg }}$. By Theorem 5.3, we then have

$$
\# \mathcal{C}(H)=\operatorname{dim} \mathrm{CF}_{*}(M, X ; H) \geq \operatorname{dim} \mathrm{HF}_{*}(M, X ; H)=\operatorname{dim} H_{*}\left(X ; \mathbb{Z}_{2}\right) .
$$

This proves the theorem.

Remark 5.11 An alternative proof of Theorem 5.3 can be given along the lines of [24], avoiding the adiabatic limit argument of Theorem 5.4. This would involve Morse-Bott exponential decay for finite energy solutions of (22) with $H=0$ on a half cylinder $[0, \infty) \times M$ respectively $(-\infty, 0] \times M$. Since $X$ is flat, such solutions converge to a point in $X$ as $s \rightarrow \pm \infty$, and one can then study solutions where this limit point lies on a gradient trajectory of a Morse function on $X$, as in [24], to obtain the desired isomorphism from Morse to Floer homology, respectively its inverse.

If $M:=S^{3}$ with the standard hypercontact structure, the Morse-Bott exponential decay as $s \rightarrow+\infty$ can be reduced to the removable singularity Theorem C.1: If $u: \mathbb{R} \times S^{3} \rightarrow X$ is a solution of (22) with $H=0$ and $w: \mathbb{H} \backslash\{0\} \rightarrow X$ is given by $w\left(e^{-s} y\right):=u(s, y)$ then

$$
\partial_{0} w-I \partial_{1} w-J \partial_{2} w-K \partial_{3} w=0 .
$$

Moreover, the energy of $w$ on a ball of radius $r=e^{-s_{0}}$ is given by

$$
r^{2} \int_{B_{r}}|d w|^{2}=\mathcal{A}\left(u\left(s_{0}, \cdot\right)\right)=2 \int_{s_{0}}^{\infty} \int_{S^{3}}\left|\partial_{S} u\right|^{2} .
$$

(Here we use $\kappa=2$ for $M=S^{3}$.) We emphasize that no such argument is available for the limit $s \rightarrow-\infty$. This reflects a fundamental asymmetry in Equation (22) related to the noncommutativity of the quaternions.

\section{Appendix A Hypercontact manifolds}

Let $M$ be an oriented 3-manifold. Three contact structures $\xi_{1}, \xi_{2}, \xi_{3}$ on $M$ are said to form a hypercontact structure if there exists a 1 -form $\alpha=\left(\alpha_{1}, \alpha_{2}, \alpha_{3}\right) \in \Omega^{1}\left(M, \mathbb{R}^{3}\right)$ such that $\alpha_{i} \wedge d \alpha_{i}>0, \xi_{i}=\operatorname{ker} \alpha_{i}$, and

$$
\alpha_{i} \wedge d \alpha_{i}=\alpha_{j} \wedge d \alpha_{j}=: \sigma, \quad \alpha_{i} \wedge d \alpha_{j}+\alpha_{j} \wedge d \alpha_{i}=0
$$

for $i \neq j$. The 1 -form $\alpha$ is determined by the contact structures $\xi_{i}$ up to multiplication by a positive function on $M$. We shall sometimes abuse notation and refer to the 
1 -form $\alpha \in \Omega^{1}\left(M, \mathbb{R}^{3}\right)$ as the hypercontact structure. Associated to $\alpha$ is a family of contact forms

$$
\alpha_{\lambda}:=\langle\lambda, \alpha\rangle=\lambda_{1} \alpha_{1}+\lambda_{2} \alpha_{2}+\lambda_{3} \alpha_{3}
$$

parametrized by the standard 2-sphere $S^{2} \subset \mathbb{R}^{3}$. In this formulation equations (66) hold if and only the volume form $\alpha_{\lambda} \wedge d \alpha_{\lambda}$ is independent of $\lambda$. Hypercontact structures were introduced and studied by Geiges-Gonzalo [13; 14]. They used the term taut contact sphere for the map $\lambda \mapsto \alpha_{\lambda}$. The term hypercontact structure was used with a different meaning in [15].

Lemma A.1 Let $\alpha \in \Omega^{1}\left(M, \mathbb{R}^{3}\right)$ be a hypercontact structure. Then the associated Reeb vector fields $v_{1}, v_{2}, v_{3} \in \operatorname{Vect}(M)$ are everywhere linearly independent.

Proof Since $\alpha_{\lambda} \wedge d \alpha_{\lambda}=|\lambda|^{2} \sigma$ for $\lambda \in \mathbb{R}^{3}$ the 2-forms $d \alpha_{1}, d \alpha_{2}, d \alpha_{3}$ are everywhere linearly independent. Since $d \alpha_{i}=\iota\left(v_{i}\right) \sigma$ this shows that $v_{1}, v_{2}, v_{3}$ are everywhere linearly independent.

Remark A.2 If the 1-forms $\alpha_{1}, \alpha_{2}, \alpha_{3}$ form a hypercontact structure then, by Lemma A.1, the Reeb vector fields $v_{1}, v_{2}, v_{3}$ form a global framing of the tangent bundle. Call the hypercontact structure positive if this framing is compatible with the orientation. This can be achieved by reversing the sign of all three 1-forms, if necessary. In the positive case the function

$$
\kappa:=d \alpha_{1}\left(v_{2}, v_{3}\right)=d \alpha_{2}\left(v_{3}, v_{1}\right)=d \alpha_{3}\left(v_{1}, v_{2}\right)
$$

on $M$ is positive. Moreover, it is convenient to choose a Riemannian metric on $M$ in which the $v_{i}$ form an orthonormal basis. The associated volume form is given by

$$
\operatorname{dvol}_{M}=\frac{\alpha_{i} \wedge d \alpha_{i}}{\kappa}, \quad i=1,2,3
$$

Remark A.3 Let $\alpha_{1}, \alpha_{2}, \alpha_{3}$ be a hypercontact structure with Reeb vector fields $v_{1}, v_{2}, v_{3}$ and, for $\lambda \in S^{2}$, denote $v_{\lambda}:=\lambda_{1} v_{1}+\lambda_{2} v_{2}+\lambda_{3} v_{3}$. Then $v_{\lambda}$ is the Reeb vector field of $\alpha_{\lambda}$. To see this note that

$$
\alpha_{i}\left(v_{j}\right)+\alpha_{j}\left(v_{i}\right)=0, \quad d \alpha_{i}\left(v_{j}, \cdot\right)+d \alpha_{j}\left(v_{i}, \cdot\right)=0
$$

for $i \neq j$, by (66) and Lemma A.1. Hence $\alpha_{\lambda}\left(v_{\lambda}\right)=1$ and $d \alpha_{\lambda}\left(v_{\lambda}, \cdot\right)=0$.

Lemma A.4 Let $\alpha$ be a hypercontact structure on $M$ with Reeb vector fields $v_{1}, v_{2}, v_{3}$. Let $\kappa: M \rightarrow \mathbb{R}$ be defined by (67) and $\mu: M \rightarrow \mathbb{R}^{3}$ by

$$
\mu_{1}:=\alpha_{2}\left(v_{3}\right), \quad \mu_{2}:=\alpha_{3}\left(v_{1}\right), \quad \mu_{3}:=\alpha_{1}\left(v_{2}\right) .
$$

Let $e_{1}, e_{2}, e_{3}$ denote the standard basis of $\mathbb{R}^{3}$. Then the following holds. 
(i) The Lie brackets of the Reeb vector fields satisfy

$$
\left[v_{2}, v_{3}\right]=\kappa v_{1}, \quad\left[v_{3}, v_{1}\right]=\kappa v_{2}, \quad\left[v_{1}, v_{2}\right]=\kappa v_{3}
$$

if and only if

$$
d \mu\left(v_{i}\right)=\kappa e_{i} \wedge \mu, \quad i=1,2,3
$$

(ii) If (69) and (70) hold then $\kappa$ is constant. Conversely, if $\kappa$ and $\mu$ are constant then $\mu \equiv 0$.

(iii) The function $\mu$ vanishes if and only if $\alpha_{i} \wedge d \alpha_{j}=0$ for $i \neq j$, or equivalently $d \alpha_{i}=\kappa * \alpha_{i}$ for $i=1,2,3$. Here $*$ denotes the Hodge $*$-operator.

Definition A.5 A positive hypercontact structure $\alpha$ with $\mu \equiv 0$ is called a Cartan structure.

Corollary A.6 If $\alpha$ is a Cartan structure then $\kappa$ is constant, the $\alpha_{i}$ form the dual basis of the $v_{i}$, the $v_{i}$ satisfy (69), $\alpha_{i} \wedge d \alpha_{j}=0$ for $i \neq j$, and $d^{*} \alpha_{i}=0$ for $i=1,2,3$.

Proof of Lemma A.4 We introduce the 1-form $\rho \in \Omega^{1}\left(M, \mathbb{R}^{3}\right)$ and the vector fields $w_{1}, w_{2}, w_{3} \in \operatorname{Vect}(M)$ by

$$
\rho:=\frac{1}{\kappa}\left(\begin{array}{c}
d \alpha_{2}\left(v_{3}, \cdot\right) \\
d \alpha_{3}\left(v_{1}, \cdot\right) \\
d \alpha_{1}\left(v_{2}, \cdot\right)
\end{array}\right), \quad \begin{aligned}
& w_{1}:=\left[v_{2}, v_{3}\right] \\
& w_{2}:=\left[v_{3}, v_{1}\right] \\
& w_{3}:=\left[v_{1}, v_{2}\right] .
\end{aligned}
$$

Then $\rho$ satisfies

$$
\rho_{i}\left(v_{j}\right)=\delta_{i j}, \quad \alpha(\xi)=\rho(\xi)+\rho(\xi) \wedge \mu
$$

We also introduce the matrices

$$
\begin{aligned}
& A:=\left(\begin{array}{lll}
\alpha_{1}\left(w_{1}\right) & \alpha_{1}\left(w_{2}\right) & \alpha_{1}\left(w_{3}\right) \\
\alpha_{2}\left(w_{1}\right) & \alpha_{2}\left(w_{2}\right) & \alpha_{2}\left(w_{3}\right) \\
\alpha_{3}\left(w_{1}\right) & \alpha_{3}\left(w_{2}\right) & \alpha_{3}\left(w_{3}\right)
\end{array}\right), \quad S:=\left(\begin{array}{lll}
\rho_{1}\left(w_{1}\right) & \rho_{1}\left(w_{2}\right) & \rho_{1}\left(w_{3}\right) \\
\rho_{2}\left(w_{1}\right) & \rho_{2}\left(w_{2}\right) & \rho_{2}\left(w_{3}\right) \\
\rho_{3}\left(w_{1}\right) & \rho_{3}\left(w_{2}\right) & \rho_{3}\left(w_{3}\right)
\end{array}\right), \\
& \Phi:=\left(\begin{array}{ccc}
1 & \mu_{3} & -\mu_{2} \\
-\mu_{3} & 1 & \mu_{1} \\
\mu_{2} & -\mu_{1} & 1
\end{array}\right), \quad B:=\left(\begin{array}{ccc}
d \mu_{1}\left(v_{1}\right) & d \mu_{1}\left(v_{2}\right) & d \mu_{1}\left(v_{3}\right) \\
d \mu_{2}\left(v_{1}\right) & d \mu_{2}\left(v_{2}\right) & d \mu_{2}\left(v_{3}\right) \\
d \mu_{3}\left(v_{1}\right) & d \mu_{3}\left(v_{2}\right) & d \mu_{3}\left(v_{3}\right)
\end{array}\right) .
\end{aligned}
$$

Then the second equation in (71) implies $\Phi S=A$. Next we observe that

$$
\alpha_{i}\left(\left[v_{j}, v_{k}\right]\right)=d \alpha_{i}\left(v_{j}, v_{k}\right)-\mathcal{L}_{v_{j}} \alpha_{i}\left(v_{k}\right)+\mathcal{L}_{v_{k}} \alpha_{i}\left(v_{j}\right)
$$


Hence $\alpha_{i}\left(\left[v_{j}, v_{k}\right]\right)=\kappa+d \mu_{j}\left(v_{j}\right)+d \mu_{k}\left(v_{k}\right)$ whenever $i, j, k$ is a cyclic permutation of $1,2,3$, and $\alpha_{i}\left(\left[v_{i}, v_{k}\right]\right)=-\mathcal{L}_{v_{i}} \alpha_{i}\left(v_{k}\right)$. These identities can be summarized in the form $\alpha_{i}\left(w_{j}\right)+d \mu_{j}\left(v_{i}\right)=\left(\kappa+\sum_{k} d \mu_{k}\left(v_{k}\right)\right) \delta_{i j}$ or

$$
\Phi S=A=\left(\kappa+\sum_{k} d \mu_{k}\left(v_{k}\right)\right) \mathbb{1}-B^{T}
$$

Moreover, we have

$$
\begin{aligned}
0= & d d \alpha_{1}\left(v_{1}, v_{2}, v_{3}\right) \\
= & \mathcal{L}_{v_{1}} d \alpha_{1}\left(v_{2}, v_{3}\right)+\mathcal{L}_{v_{2}} d \alpha_{1}\left(v_{3}, v_{1}\right)+\mathcal{L}_{v_{3}} d \alpha_{1}\left(v_{1}, v_{2}\right) \\
& -d \alpha_{1}\left(v_{1},\left[v_{2}, v_{3}\right]\right)-d \alpha_{1}\left(v_{2},\left[v_{3}, v_{1}\right]\right)-d \alpha_{1}\left(v_{3},\left[v_{1}, v_{2}\right]\right) \\
= & d \kappa\left(v_{1}\right)-d \alpha_{1}\left(v_{2},\left[v_{3}, v_{1}\right]\right)-d \alpha_{1}\left(v_{3},\left[v_{1}, v_{2}\right]\right)
\end{aligned}
$$

Repeating the argument for $\alpha_{2}$ and $\alpha_{3}$ and using Equation (68) we obtain

$$
\begin{aligned}
& d \kappa\left(v_{1}\right)=\kappa\left(\rho_{3}\left(w_{2}\right)-\rho_{2}\left(w_{3}\right)\right), \\
& d \kappa\left(v_{2}\right)=\kappa\left(\rho_{1}\left(w_{3}\right)-\rho_{3}\left(w_{1}\right)\right), \\
& d \kappa\left(v_{3}\right)=\kappa\left(\rho_{2}\left(w_{1}\right)-\rho_{1}\left(w_{2}\right)\right),
\end{aligned}
$$

Hence $\kappa$ is constant if and only if the matrix $S$ is symmetric.

We prove (i). Equation (69) is equivalent to $S=\kappa \mathbb{1}$ and Equation (70) to $B=\kappa(\Phi-\mathbb{1}$ ). If $S=\kappa \mathbb{1}$ then it follows from (72) that

$$
B^{T}=\kappa(\mathbb{1}-\Phi)+\sum_{k} d \mu_{k}\left(v_{k}\right) \mathbb{1}
$$

Examining the diagonal entries we find that $d \mu_{k}\left(v_{k}\right)=0$ for $k=1,2,3$ and hence $B^{T}=\kappa(\mathbb{1}-\Phi)$. This in turn implies that $B^{T}=-B$ and thus $B=\kappa(\Phi-\mathbb{1})$. Conversely, if $B=\kappa(\Phi-\mathbb{1})$ then $B$ is skew symmetric and $d \mu_{k}\left(v_{k}\right)=0$ for all $k$. So it follows from (72) that $\Phi S=\kappa \mathbb{1}+B=\kappa \Phi$ and hence $S=\kappa \mathbb{1}$.

We prove (ii). If (69) holds then $S=\kappa \mathbb{1}$ is symmetric and so $\kappa$ is constant, by (73). Conversely, if $\kappa$ and $\mu$ are constant then, by (72), we have $\Phi S=\kappa \mathbb{1}$ and, by (73), $S=S^{T}$. Hence $\Phi$ is symmetric and so $\mu \equiv 0$.

To prove (iii) we observe that, for every cyclic permutation $i, j, k$ of $1,2,3$, we have $\alpha_{i} \wedge d \alpha_{j}=\kappa \mu_{k} \operatorname{dvol}_{M}$ and $\kappa * \alpha_{i}=d \alpha_{i}+\mu_{k} d \alpha_{j}-\mu_{j} d \alpha_{k}$. (Take the product with a 1 -form $\beta$ and use the identity $\left(\beta \wedge d \alpha_{i}\right)\left(v_{1}, v_{2}, v_{3}\right)=\kappa \beta\left(v_{i}\right)$.) This proves the lemma. 
Example A.7 The standard hypercontact structure on the unit sphere $S^{3} \subset \mathbb{R}^{4}$ with coordinates $y=\left(y_{0}, y_{1}, y_{2}, y_{3}\right)$ is given by the 1 -forms

$$
\begin{aligned}
& \alpha_{1}:=y_{0} d y_{1}-y_{1} d y_{0}+y_{2} d y_{3}-y_{3} d y_{2}, \\
& \alpha_{2}:=y_{0} d y_{2}-y_{2} d y_{0}+y_{3} d y_{1}-y_{1} d y_{3}, \\
& \alpha_{3}:=y_{0} d y_{3}-y_{3} d y_{0}+y_{1} d y_{2}-y_{2} d y_{1} .
\end{aligned}
$$

Identify $\mathbb{R}^{4}$ with the quaternions via

$$
y=y_{0}+\mathbf{i} y_{1}+\mathbf{j} y_{2}+\mathbf{k} y_{3}
$$

and $\mathbb{R}^{3}$ with the imaginary quaternions via $\lambda=\mathbf{i} \lambda_{1}+\mathbf{j} \lambda_{2}+\mathbf{k} \lambda_{3}$. Then the 1 -form $\alpha_{\lambda}:=\lambda_{1} \alpha_{1}+\lambda_{2} \alpha_{2}+\lambda_{3} \alpha_{3}$ and its Reeb vector field $v_{\lambda}$ are given by

$$
\alpha_{\lambda}(y ; \eta)=\operatorname{Re}(\lambda y \bar{\eta}), \quad v_{\lambda}(y)=\lambda y
$$

for $\lambda \in S^{2} \subset \operatorname{Im}(\mathbb{H})$ and $\eta \in T_{y} S^{3}$. We emphasize that in this example $\mu \equiv 0$ and $\kappa \equiv 2$.

The standard hypercontact structure on $S^{3}$ is preserved by the right action of the unit quaternions via $\operatorname{Sp}(1) \times S^{3} \rightarrow S^{3}:(a, y) \mapsto y a$. For the left action of $\operatorname{Sp}(1)$ on $S^{3}$ we have

$$
\phi_{a}^{*} \alpha_{\lambda}=\alpha_{a^{-1} \lambda a}, \quad \phi_{a}^{*} v_{\lambda}=v_{a^{-1} \lambda a}
$$

where $\phi_{a} \in \operatorname{Diff}\left(S^{3}\right)$ is given by $\phi_{a}(y):=a y$ for $a \in \operatorname{Sp}(1)$ and $y \in S^{3}$.

Proposition A.8 (Geiges-Gonzalo [13; 14]) Every Cartan hypercontact 3-manifold $(M, \alpha)$ is diffeomorphic to a quotient of the 3-sphere (with the standard hypercontact structure up to scaling) by a finite subgroup of $\mathrm{Sp}(1)$.

Proof By rescaling, if necessary, we may assume that $\kappa=2$. Then there is a unique Lie algebra homomorphism $\operatorname{Lie}(\operatorname{Sp}(1))=\operatorname{Im}(\mathbb{H}) \rightarrow \operatorname{Vect}(M): \xi \mapsto v_{\xi}$ such that $v_{\mathbf{i}}, v_{\mathbf{j}}, v_{\mathbf{k}}$ are the Reeb vector fields of $\alpha_{1}, \alpha_{2}, \alpha_{3}$, respectively. Since $M$ is compact and $\mathrm{Sp}(1)$ is simply connected, this Lie algebra homomorphism integrates to a unique Lie group homomorphism

$$
\operatorname{Sp}(1) \rightarrow \operatorname{Diff}(M): x \mapsto \phi_{x} .
$$

This group action of $\mathrm{Sp}(1)$ on $M$ is transitive, because $M$ is connected, and it has finite isotropy subgroups. Fix an element $y_{0} \in M$ and define the map $\psi: \operatorname{Sp}(1) \rightarrow M$ by $\psi(x):=\phi_{x}\left(y_{0}\right)$. This map induces a diffeomorphism $\mathrm{Sp}(1) / \mathrm{G}_{0} \rightarrow M$, where $\mathrm{G}_{0}:=\left\{x \in \mathrm{Sp}(1) \mid \phi_{x}\left(y_{0}\right)=y_{0}\right\}$ denotes the stabilizer of $y_{0}$. This diffeomorphism identifies the vector field $x \mapsto \mathbf{i} x$ on $\mathrm{Sp}(1) / \mathrm{G}_{0}$ with the vector field $v_{\mathbf{i}}$ on $M$ and similarly for $\mathbf{j}$ and $\mathbf{k}$. 


\section{Appendix B The Heinz trick for subcritical exponents}

Let $M$ be a smooth Riemannian $n$-manifold (not necessarily compact) and let $\mathcal{L}$ be a scalar second order elliptic operator. We assume that $\mathcal{L}$ differs from the Laplace Beltrami operator $\Delta:=-d^{*} d$ by a first order operator. We study nonnegative solutions $e: M \rightarrow \mathbb{R}$ of the differential inequality

$$
\begin{gathered}
\mathcal{L} e \geq-A-B e^{\mu} \\
1 \leq \mu \leq \frac{n+2}{n} .
\end{gathered}
$$

where

In the critical case $\mu=(n+2) / n$ the Heinz trick gives a mean value inequality for nonnegative solutions $e: B_{r}\left(p_{0}\right) \rightarrow[0, \infty)$ of (74) with sufficiently small $L^{1}$ norm (see for example Robbin-Salamon [26] and Wehrheim [30]). For $\mu<(n+2) / n$ the same proof shows that the condition on the $L^{1}$ norm can be dropped and one obtains a global estimate for the sup-norm in terms of the $L^{1}$ norm of $e$.

Theorem B.1 Let $K \subset M$ be a compact set and let $1 \leq \mu \leq(n+2) / n$.

(i) Assume $\mu<(n+2) / n$. Then there is a constant $c>0$ with the following significance. If $e: M \rightarrow \mathbb{R}$ is a nonnegative $C^{2}$ function satisfying (74) then

$$
\sup _{K} e \leq c\left(A+\int_{M} e \operatorname{dvol}_{M}+\left(B^{n / 2} \int_{M} e \mathrm{dvol}_{M}\right)^{\alpha}\right),
$$

where $\alpha:=2 /(2+n-n \mu)$.

(ii) Assume $\mu=(n+2) / n$. Then there are positive constants $\hbar, \delta, c$ with the following significance. If $e: M \rightarrow \mathbb{R}$ is a nonnegative $C^{2}$ function satisfying (74) then, for $x \in K$ and $0<r \leq \delta$, we have

$$
B^{n / 2} \int_{B_{r}(x)} e<\hbar \quad \Longrightarrow \quad e(x) \leq c\left(A r^{2}+\frac{1}{r^{n}} \int_{B_{r}(x)} e \operatorname{dvol}_{M}\right) .
$$

Proof The proof has three steps. For $p_{0} \in M$ and $r>0$ we denote by $B_{r}\left(p_{0}\right) \subset M$ the closed ball of radius $r$ about $p_{0}$. The first step restates Theorem 9.20 in [16].

Step 1 There are constants $c_{1}>0$ and $\delta>0$ with the following significance. If $p_{0} \in K$ and $0<r \leq \delta$ then every nonnegative $C^{2}$ function $e: B_{r}\left(p_{0}\right) \rightarrow \mathbb{R}$ satisfies

$$
\Delta e \geq 0 \quad \Longrightarrow \quad e\left(p_{0}\right) \leq \frac{c_{1}}{r^{n}} \int_{B_{r}\left(p_{0}\right)} e \operatorname{dvol}_{M}
$$


Step 2 There are constants $c_{2}>0$ and $\delta>0$ with the following significance. If $p_{0} \in K, 0<r \leq \delta$, and $A \geq 0$, then every nonnegative $C^{2}$ function $e: B_{r}\left(p_{0}\right) \rightarrow \mathbb{R}$ satisfies

$$
\mathcal{L} e \geq-A \quad \Longrightarrow \quad e\left(p_{0}\right) \leq c_{2}\left(A r^{2}+\frac{1}{r^{n}} \int_{B_{r}\left(p_{0}\right)} e \mathrm{dvol}_{M}\right) .
$$

Let $\delta$ be smaller than the injectivity radius of $M$ and than the constant in Step 1 . Choose geodesic coordinates $y^{1}, y^{2}, \ldots, y^{n}$ in $B_{r}\left(y_{0}\right)$ with $y^{i}\left(p_{0}\right)=0$. Then

$$
\mathcal{L}=\sum_{\mu, v} a^{\mu \nu} \frac{\partial^{2}}{\partial y^{\mu} \partial y^{v}}+\sum_{\nu} b^{v} \frac{\partial}{\partial y^{v}}
$$

with $a^{\mu \nu}(0)=\delta^{\mu \nu}$. Choose $\delta$ so small that

$$
|y| \leq \delta \quad \Longrightarrow \quad\left|a^{v v}(y)-1\right|+\delta\left|b^{v}(y)\right| \leq \frac{1}{n}
$$

for $v=1, \ldots, n$. Denote by $\Delta_{0}=\sum_{v}\left(\frac{\partial}{\partial y^{v}}\right)^{2}$ the standard Laplace operator and consider the function

$$
u(y):=\frac{A}{2}|y|^{2} .
$$

This function satisfies $\Delta_{0} u=n A$ and

$$
\left(\mathcal{L} u-\Delta_{0} u\right)(y)=A\left(\sum_{v}\left(a^{\nu v}(y)-1\right)+\sum_{v} b^{v}(y) y^{v}\right) \geq-A .
$$

Hence $\mathcal{L} u \geq(n-1) A$ and

$$
\mathcal{L}(e+u) \geq \mathcal{L} e+(n-1) A \geq 0 .
$$

By Step 1, this implies

$$
e(0)=e(0)+u(0) \leq \frac{c_{1}}{r^{n}} \int_{B_{r}}(e+u) \operatorname{dvol}_{M} .
$$

Hence the assertion follows from the fact that

$$
\int_{B_{r}} u \operatorname{dvol}_{M} \leq \omega_{n} A \int_{0}^{r} \rho^{n+1} d \rho=\frac{\omega_{n} A r^{n+2}}{n+2} .
$$

Here $\omega_{n}$ denotes the area of the unit sphere in $\mathbb{R}^{n}$ and $\delta$ is chosen so small that $\operatorname{dvol}_{M}$ and the volume form of the flat metric differ by a factor at most 2 . This proves Step 2. 
Step 3 There is a constant $c_{3}>0$ with the following significance. If $e: M \rightarrow \mathbb{R}$ is a nonnegative $C^{2}$ function satisfying

$$
\mathcal{L} e \geq-A-B e^{\mu}
$$

for some constants $A, B \geq 0$ then

$$
\sup _{K} e \leq c_{3}\left(A+\int_{M} e \operatorname{dvol}_{M}+\left(B^{n / 2} \int_{M} e \operatorname{dvol}_{M}\right)^{2 /(2+n-n \mu)}\right) .
$$

Let $\delta$ be as in Step 2 and assume $c_{2} \delta^{2} \leq \frac{1}{4}$. Fix a point $p_{0} \in K$. Define $h:[0, \delta] \rightarrow \mathbb{R}$ by

$$
h(s):=\left(\frac{\delta-s}{\delta}\right)^{n} \max _{B_{s}\left(p_{0}\right)} e .
$$

Then

$$
h(0)=e\left(p_{0}\right), \quad h(\delta)=0 .
$$

Since $h$ is nonnegative there is an $s^{*} \in[0, \delta)$ and a $p^{*} \in B_{s^{*}}\left(p_{0}\right)$ such that

$$
h\left(s^{*}\right)=\max _{0 \leq s \leq \delta} h(s), \quad c:=e\left(p^{*}\right)=\max _{B_{s^{*}}\left(p_{0}\right)} e .
$$

Denote

$$
\varepsilon:=\frac{\delta-s^{*}}{2}
$$

Then $\max _{B_{\varepsilon}\left(p^{*}\right)} e \leq \max _{B_{s^{*}+\varepsilon}\left(p_{0}\right)} e=\frac{\delta^{n} h\left(s^{*}+\varepsilon\right)}{\left(\delta-s^{*}-\varepsilon\right)^{n}} \leq \frac{2^{n} \delta^{n} h\left(s^{*}\right)}{\left(\delta-s^{*}\right)^{n}}=2^{n} \max _{B_{s^{*}}\left(p_{0}\right)} e=2^{n} c$.

Hence in $B_{\varepsilon}\left(p^{*}\right)$ we have the inequality

$$
\mathcal{L} e \geq-A-B e^{\mu} \geq-A-B\left(2^{n} c\right)^{\mu} .
$$

By Step 2 this implies

$$
c=e\left(p^{*}\right) \leq c_{2}\left(\left(A+B\left(2^{n} c\right)^{\mu}\right) r^{2}+\frac{1}{r^{n}} \int_{M} e \operatorname{dvol}_{M}\right)
$$

for $0 \leq r \leq \varepsilon$. Now comes the crucial case distinction.

Case 1 If $c \leq A$ then we have

$$
e\left(p_{0}\right) \leq c \leq A
$$

and so the desired estimate holds with $c_{3}=1$. Thus we may assume $A \leq c$.

Case 2 Assume

$$
A \leq c, \quad c_{2} B 2^{n \mu} c^{\mu-1} \varepsilon^{2} \geq \frac{1}{4}
$$


Then we may choose $r \leq \varepsilon<\delta$ such that $c_{2} B 2^{n \mu} c^{\mu-1} r^{2}=\frac{1}{4}$ and obtain

$$
c_{2}\left(A+B\left(2^{n} c\right)^{\mu}\right) r^{2} \leq c_{2} c \delta^{2}+c_{2} B\left(2^{n} c\right)^{\mu} r^{2} \leq \frac{c}{2} .
$$

Hence, by (77), we have

$$
c \leq \frac{2 c_{2}}{r^{n}} \int_{M} e \operatorname{dvol}_{M}=2 c_{2}\left(4 c_{2} B 2^{n \mu}\right)^{n / 2} c^{(n \mu-n) / 2} \int_{M} e \operatorname{dvol}_{M} .
$$

Since $\mu<(n+2) / n$ we have $2+n-n \mu>0$ and hence

$$
e\left(p_{0}\right) \leq c \leq c_{3}\left(B^{n / 2} \int_{M} e \operatorname{dvol}_{M}\right)^{2 /(2+n-n \mu)},
$$

with $c_{3}:=\left(2 c_{2}\left(c_{2} 2^{n \mu+2}\right)^{n / 2}\right)^{2 /(2+n-n \mu)}$. (For the critical exponent we have that $(n \mu-n) / 2=1$. In this situation Case 2 can be excluded by the assumption of a sufficiently small upper bound on $B^{n / 2} \int e \operatorname{dvol}_{M}$.)

Case 3 Assume

$$
A \leq c, \quad c_{2} B 2^{n \mu} c^{\mu-1} \varepsilon^{2}<\frac{1}{4} .
$$

Then we may choose $r=\varepsilon$ and obtain $c_{2}\left(A+B\left(2^{n} c\right)^{\mu}\right) \varepsilon^{2} \leq \frac{c}{2}$ as before. Hence, by (77), we have

$$
c \leq \frac{2 c_{2}}{\varepsilon^{n}} \int_{M} e \operatorname{dvol}_{M} .
$$

Since $\delta-s^{*}=2 \varepsilon$ this gives

$$
e\left(p_{0}\right)=h(0) \leq h\left(s^{*}\right)=c\left(\frac{\delta-s^{*}}{\delta}\right)^{n}=\frac{2^{n} c \varepsilon^{n}}{\delta^{n}} \leq \frac{2^{n+1} c_{2}}{\delta^{n}} \int_{M} e \operatorname{dvol}_{M} .
$$

Thus in this case the estimate of Step 3 holds with $c_{3}=2^{n+1} c_{2} / \delta^{n}$. This proves the theorem.

\section{Appendix C A removable singularity theorem}

Denote by $B \subset \mathbb{R}^{4}$ the unit ball with coordinates $t=\left(t_{0}, t_{1}, t_{2}, t_{3}\right)$ and by

$$
B_{r}:=\left\{t \in \mathbb{R}^{4}|| t \mid \leq r\right\}, \quad S_{r}:=\left\{t \in \mathbb{R}^{4}|| t \mid=r\right\},
$$

the ball and sphere of radius $r$. Let $X$ be a hyperkähler manifold with complex structures $I, J, K$. Let $w: B \rightarrow \operatorname{Vect}(X)$ and $\Xi=\left(\xi_{i}^{j}\right): B \rightarrow \mathbb{R}^{4 \times 4}$ be smooth maps 
such that $\Xi(0)=\mathbb{1}$ is the identity matrix and $\Xi(t)$ is nonsingular for every $t \in B$. We examine solutions of the equation

$$
\sum_{i=0}^{3}\left(\xi_{0}^{i}(t) \partial_{i} u+\xi_{1}^{i}(t) I \partial_{i} u+\xi_{2}^{i}(t) J \partial_{i} u+\xi_{3}^{i}(t) K \partial_{i} u\right)=\nabla w(t, u) .
$$

Associated to Equation (78) is the elliptic operator

$$
L:=\sum_{i, j=0}^{k} a^{i j} \partial_{i} \partial_{j}+\sum_{j=0}^{3} b^{j} \partial_{j}, \quad a^{i j}:=\sum_{v} \xi_{v}^{i} \xi_{v}^{j}, \quad b^{j}:=\sum_{v, i}\left(\partial_{i} \xi_{v}^{j}\right) \xi_{v}^{i} .
$$

Theorem C.1 Assume $X$ is a compact flat hyperkähler manifold (possibly with boundary). If $u: B \backslash\{0\} \rightarrow X$ is a solution of (78) on the punctured disc and

$$
\int_{B}|d u|^{2}=\sum_{i=0}^{3} \int_{B}\left|\partial_{i} u\right|^{2}<\infty
$$

then $u$ extends to a smooth function from $B$ to $X$.

Remark C.2 In Theorem C.1 the condition that $X$ is flat cannot be omitted. For example, let $f: S^{3} \rightarrow X$ be a nonconstant critical point of the hypersymplectic action functional $\mathcal{A}$. Such critical points are described in the introduction (compositions of rational curves with Hopf fibrations) and they do not exist in the flat case, by Lemma 3.7. Identify $S^{3}$ with the unit sphere in $\mathbb{H}$ and define $u: \mathbb{H} \backslash\{0\} \rightarrow X$ by

$$
u(t):=f\left(|t|^{-1} t\right) .
$$

Then $u$ satisfies the equation $\partial_{0} u-I \partial_{1} u-J \partial_{2} u-K \partial_{3} u=0$. Moreover, we have $|d u(t)|^{2}=|t|^{-2}\left|d f\left(|t|^{-1} t\right)\right|^{2}$ and hence

$$
\int_{B_{r}}|d u|^{2}=\frac{r^{2}}{2} \int_{S^{3}}|d f|^{2}=r^{2} \mathcal{A}(f)
$$

for every $r>0$. However, the singularity of $u$ at the origin cannot be removed.

Lemma C.3 Assume $X$ is a compact flat hyperkähler manifold. Then there is a constant $C>0$ with the following significance. If $u: B \backslash\{0\} \rightarrow X$ is a solution of (78) then the function $e=e_{u}: B \rightarrow[0, \infty)$ defined by

$$
e(t):=\frac{1}{2} \sum_{j=0}^{3}\left|\sum_{i=0}^{3} \xi_{j}^{i}(t) \partial_{i} u(t)\right|^{2}
$$

satisfies the inequality

$$
L e \geq-C\left(1+e^{3 / 2}\right) .
$$


Proof The proof uses word by word the same arguments as in Lemma 3.3 and will be omitted.

The exponent $\frac{3}{2}=\frac{n+2}{n}$ in Lemma C.3 is the critical exponent of Theorem B. 1 for $n=4$. Hence every solution $u: B \backslash\{0\} \rightarrow X$ of (78) satisfies an inequality of the form

$$
|t|=r \quad \Longrightarrow \quad|d u(t)|^{2} \leq c r^{2}+\frac{c}{r^{4}} \int_{B_{2 r}}|d u|^{2}
$$

for $r$ sufficiently small and a suitable constant $c$. Thus $|t|^{4}|d u(t)|^{2}$ converges to zero as $t$ tends to zero.

It is convenient to introduce the 1 -forms $\theta_{1}, \theta_{2}, \theta_{3}$ and the vector fields $v_{0}, v_{1}, v_{2}, v_{3}$ on $B$ by

$$
\begin{aligned}
& \theta_{1}:=t_{0} d t_{1}-t_{1} d t_{0}-t_{2} d t_{3}+t_{3} d t_{2}, \\
& \theta_{2}:=t_{0} d t_{2}-t_{2} d t_{0}-t_{3} d t_{1}+t_{1} d t_{3}, \\
& \theta_{3}:=t_{0} d t_{3}-t_{3} d t_{0}-t_{1} d t_{2}+t_{2} d t_{1}, \\
& v_{0}:=t_{0} \partial_{0}+t_{1} \partial_{1}+t_{2} \partial_{2}+t_{3} \partial_{3}, \\
& v_{1}:=t_{0} \partial_{1}-t_{1} \partial_{0}-t_{2} \partial_{3}+t_{3} \partial_{2}, \\
& v_{2}:=t_{0} \partial_{2}-t_{2} \partial_{0}-t_{3} \partial_{1}+t_{1} \partial_{3}, \\
& v_{3}:=t_{0} \partial_{3}-t_{3} \partial_{0}-t_{1} \partial_{2}+t_{2} \partial_{1} .
\end{aligned}
$$

Note that the $v_{i}$ are orthogonal and $\left|v_{i}(t)\right|=|t|$. In particular, for $t \in S_{r}$ the vectors $r^{-1} v_{1}(t), r^{-1} v_{2}(t), r^{-1} v_{3}(t)$ form an orthonormal basis of the tangent space $T_{t} S_{r}=$ $t^{\perp}$. The energy and the hypersymplectic action of a smooth map $u: S_{r} \rightarrow X$ are defined by

$$
\mathcal{E}_{r}(u):=\frac{1}{r^{2}} \int_{S_{r}} \sum_{i=1}^{3}\left|d u\left(v_{i}\right)\right|^{2}, \quad \mathcal{A}_{r}(u):=\int_{S_{r}} \sum_{i} \theta_{i} \wedge u^{*} \omega_{i} .
$$

Lemma C.4 The energy and hypersymplectic action satisfy the isoperimetric inequality

$$
\mathcal{A}_{r}(u) \leq r \mathcal{E}_{r}(u)
$$

and the energy identities

$$
\mathcal{E}_{r}(u)+\frac{2}{r} \mathcal{A}_{r}(u)=\frac{1}{r^{2}} \int_{S_{r}}\left|I d u\left(v_{1}\right)+J d u\left(v_{2}\right)+K d u\left(v_{3}\right)\right|^{2} \mathrm{dvol}_{S_{r}}
$$


for every smooth map $u: S_{r} \rightarrow X$ and

$$
\int_{B_{r}}|d u|^{2}=\mathcal{A}_{r}(u)+\int_{B_{r}}\left|\partial_{0} u+I \partial_{1} u+J \partial_{2} u+K \partial_{3} u\right|^{2}
$$

for every smooth map $u$ : $B_{r} \backslash\{0\} \rightarrow X$ satisfying $\lim _{t \rightarrow 0}|t|^{4}|d u(t)|^{2}=0$.

Proof We have $\theta_{i}\left(v_{j}\right)=r^{2} \delta_{i j}$ and so the standard volume form on $S_{r}$ is dvol $S_{r}=$ $r^{-3} \theta_{1} \wedge \theta_{2} \wedge \theta_{3}$. Hence $\theta_{i} \wedge u^{*} \omega_{i}=r^{-1} u^{*} \omega_{i}\left(v_{j}, v_{k}\right) \mathrm{dvol}_{S_{r}}$ for every cyclic permutation $i, j, k$ of $1,2,3$. This implies

$$
\mathcal{A}_{r}(u)=\frac{1}{r} \int_{S_{r}}\left(u^{*} \omega_{1}\left(v_{2}, v_{3}\right)+u^{*} \omega_{2}\left(v_{3}, v_{1}\right)+u^{*} \omega_{3}\left(v_{1}, v_{2}\right)\right) \operatorname{dvol}_{S_{r}}
$$

and hence the isoperimetric inequality (80). The energy identity (81) is an adaptation of Lemma 2.2 to the present notation. To prove (82) we assume that $u: B_{r} \backslash\{0\} \rightarrow X$ satisfies $\lim _{t \rightarrow 0}|t|^{4}|d u(t)|^{2}=0$. Then it follows from (80) that $\lim _{\rho \rightarrow 0} \mathcal{A}_{\rho}(u)=0$. Moreover, by direct computation, we have

$$
\int_{B_{r} \backslash B_{\rho}}\left(|d u|^{2}-\left|\partial_{0} u+I \partial_{1} u+J \partial_{2} u+K \partial_{3} u\right|^{2}\right)=\mathcal{A}_{r}(u)-\mathcal{A}_{\rho}(u)
$$

for $0<\rho \leq r$. The assertion follows by taking the limit $\rho \rightarrow 0$. This proves the lemma.

Lemma C.5 Assume $X$ is compact and fix any real number $0<\mu<4$. Let $u: B \backslash\{0\} \rightarrow X$ be a solution of (78) satisfying $\lim _{t \rightarrow 0}|t|^{4}|d u(t)|^{2}=0$. Then there are positive constants $r_{0}$ and $c$ such that

$$
0<r \leq r_{0} \quad \Longrightarrow \quad \int_{B_{r}}|d u|^{2} \leq c r^{\mu}
$$

Proof Since $\Xi(0)$ is the identity matrix, there is a constant $C>0$ such that every solution of (78) satisfies the estimate

$$
\left|\partial_{0} u(t)+I \partial_{1} u(t)+J \partial_{2} u(t)+K \partial_{3} u(t)\right|^{2} \leq C^{2}\left(|t|^{2}|d u(t)|^{2}+1\right) .
$$

Combining this with (82) we obtain

$$
\begin{aligned}
\int_{B_{r}}|d u|^{2} & =\mathcal{A}_{r}(u)+\int_{B_{r}}\left|\partial_{0} u+I \partial_{1} u+J \partial_{2} u+K \partial_{3} u\right|^{2} \\
& \leq \mathcal{A}_{r}(u)+C^{2} r^{2} \int_{B_{r}}|d u|^{2}+C^{2} \operatorname{Vol}(B) r^{4} .
\end{aligned}
$$


Since

$$
\left|d u\left(v_{0}\right)+I d u\left(v_{1}\right)+J d u\left(v_{2}\right)+K d u\left(v_{3}\right)\right|=r\left|\partial_{0} u+I \partial_{1} u+J \partial_{2} u+K \partial_{3} u\right|
$$

on $S_{r}$ and $r^{2}|d u|^{2}+1 \leq(r|d u|+1)^{2}$, it follows also from (83) that

$$
\begin{aligned}
\left|d u\left(v_{0}\right)\right| & \geq\left|I d u\left(v_{1}\right)+J d u\left(v_{2}\right)+K d u\left(v_{3}\right)\right|-C r^{2}|d u|-C r \\
\left|d u\left(v_{0}\right)\right|^{2} & \geq\left|I d u\left(v_{1}\right)+J d u\left(v_{2}\right)+K d u\left(v_{3}\right)\right|^{2}-6 C r^{3}|d u|^{2}-6 C r^{2}|d u| .
\end{aligned}
$$

This implies

$$
\begin{aligned}
\int_{S_{r}}|d u|^{2}= & \frac{1}{r^{2}} \int_{S_{r}} \sum_{i=0}^{3}\left|d u\left(v_{i}\right)\right|^{2}=\mathcal{E}_{r}(u)+\frac{1}{r^{2}} \int_{S_{r}}\left|d u\left(v_{0}\right)\right|^{2} \\
\geq & \mathcal{E}_{r}(u)+\frac{1}{r^{2}} \int_{S_{r}}\left|I d u\left(v_{1}\right)+J d u\left(v_{2}\right)+K d u\left(v_{3}\right)\right|^{2} \\
& -6 C r \int_{S_{r}}|d u|^{2}-6 C \int_{S_{r}}|d u| \\
\geq & 2 \mathcal{E}_{r}(u)+\frac{2}{r} \mathcal{A}_{r}(u)-6 C(r+\delta) \int_{S_{r}}|d u|^{2}-\frac{3 C}{2 \delta} \int_{S_{r}} 1 .
\end{aligned}
$$

Here we have dropped the volume form $\mathrm{dvol}_{S_{r}}$ in the notation. The last step follows from (81). Since $\mathcal{E}_{r}(u) \geq r^{-1} \mathcal{A}_{r}(u)$ and the area of the 3 -sphere is $4 \operatorname{Vol}(B)$ this gives

$$
(1+6 C(r+\delta)) \int_{S_{r}}|d u|^{2} \geq \frac{4}{r} \mathcal{A}_{r}(u)-\frac{6 C \operatorname{Vol}(B) r^{3}}{\delta} .
$$

On the other hand, by (84) we have

$$
\left(1-C^{2} r^{2}\right) \int_{B_{r}}|d u|^{2} \leq \mathcal{A}_{r}(u)+C^{2} \operatorname{Vol}(B) r^{4} .
$$

Combining these two inequalities we obtain

$$
\int_{B_{r}}|d u|^{2} \leq \frac{1+6 C(r+\delta)}{1-C^{2} r^{2}} \frac{r}{4} \int_{S_{r}}|d u|^{2}+\left(\frac{C^{2}}{1-C^{2} r^{2}}+\frac{3 C}{2 \delta}\right) \operatorname{Vol}(B) r^{4}
$$

for $r<1 / C$. Choose $\delta$ so small that $(1+6 C \delta) \mu<4$. Then, for $r$ sufficiently small and a suitable constant $c>0$, we have

$$
\int_{B_{r}}|d u|^{2} \leq r \mu^{-1} \int_{S_{r}}|d u|^{2}+c r^{4} .
$$


Define the function $\phi:(0,1] \rightarrow \mathbb{R}$ by

$$
\phi(r):=r^{-\mu} \int_{B_{r}}|d u|^{2}+\frac{\mu c}{4-\mu} r^{4-\mu} .
$$

Then the derivative of $\phi$ is

$$
\begin{aligned}
\frac{d}{d r} \phi(r) & =r^{-\mu} \int_{S_{r}}|d u|^{2}-\mu r^{-\mu-1} \int_{B_{r}}|d u|^{2}+\mu c r^{3-\mu} \\
& =\mu r^{-\mu-1}\left(r \mu^{-1} \int_{S_{r}}|d u|^{2}-\int_{B_{r}}|d u|^{2}+c r^{4}\right) \geq 0 .
\end{aligned}
$$

The last inequality follows from (85) and holds for $r$ sufficiently small, say for $0<$ $r \leq r_{0}$. Hence

$$
\int_{B_{r}}|d u|^{2} \leq \phi(r) r^{\mu} \leq \phi\left(r_{0}\right) r^{\mu}
$$

for $0<r \leq r_{0}$. This proves the lemma.

Proof of Theorem C.1 Choose a real number $\mu$ such that $2<\mu<4$. Combining Lemma C.5 with the inequality (79) we obtain

$$
|d u(t)|^{2} \leq \frac{c}{|t|^{4-\mu}}
$$

for a suitable constant $c>0$. For $4<p<8 /(4-\mu)$ this implies

$$
\int_{B}|d u|^{p}=\int_{0}^{1} \int_{S_{r}}|d u|^{p} \leq 4 \operatorname{Vol}(B) c^{p} \int_{0}^{1} r^{3-(4-\mu) p / 2} d r<\infty .
$$

That the integral is finite follows from the fact that $3-\frac{1}{2}(4-\mu) p>-1$. By the Sobolev embedding theorem our function $u: B \backslash\{0\} \rightarrow X$ is Hölder continuous and extends to a $W^{1, p}$ function on $B$. Now it follows from the standard elliptic bootstrapping techniques that the extended function $u$ is smooth. This proves the theorem.

\section{References}

[1] N Aronszajn, A unique continuation theorem for solutions of elliptic partial differential equations or inequalities of second order, J. Math. Pures Appl. (9) 36 (1957) 235-249 MR0092067

[2] MF Atiyah, V K Patodi, I M Singer, Spectral asymmetry and Riemannian geometry. III, Math. Proc. Cambridge Philos. Soc. 79 (1976) 71-99 MR0397799

[3] T D Cochran, N Habegger, On the homotopy theory of simply connected four manifolds, Topology 29 (1990) 419-440 MR1071367 
[4] C C Conley, E Zehnder, The Birkhoff-Lewis fixed point theorem and a conjecture of VI Arnol' d, Invent. Math. 73 (1983) 33-49 MR707347

[5] S K Donaldson, Complex cobordism, Ashtekar's equations and diffeomorphisms, from: "Symplectic geometry", (D Salamon, editor), London Math. Soc. Lecture Note Ser. 192, Cambridge Univ. Press (1993) 45-55 MR1297129

[6] S K Donaldson, Floer homology groups in Yang-Mills theory, Cambridge Tracts in Math. 147, Cambridge Univ. Press (2002) MR1883043 With the assistance of M Furuta and D Kotschick

[7] S K Donaldson, R P Thomas, Gauge theory in higher dimensions, from: "The geometric universe (Oxford, 1996)", (S A Huggett, L J Mason, K P Tod, S T Tsou, N M J Woodhouse, editors), Oxford Univ. Press, Oxford (1998) 31-47 MR1634503

[8] S Dostoglou, D A Salamon, Self-dual instantons and holomorphic curves, Ann. of Math. (2) 139 (1994) 581-640 MR1283871

[9] A Floer, An instanton-invariant for 3-manifolds, Comm. Math. Phys. 118 (1988) 215-240 MR956166

[10] A Floer, The unregularized gradient flow of the symplectic action, Comm. Pure Appl. Math. 41 (1988) 775-813 MR948771

[11] A Floer, Symplectic fixed points and holomorphic spheres, Comm. Math. Phys. 120 (1989) 575-611 MR987770

[12] A Floer, H Hofer, D Salamon, Transversality in elliptic Morse theory for the symplectic action, Duke Math. J. 80 (1995) 251-292 MR1360618

[13] H Geiges, J Gonzalo, Contact geometry and complex surfaces, Invent. Math. 121 (1995) 147-209 MR1345288

[14] H Geiges, J Gonzalo Pérez, Contact spheres and hyperkähler geometry, Comm. Math. Phys. 287 (2009) 719-748 MR2481757

[15] H Geiges, C B Thomas, Hypercontact manifolds, J. London Math. Soc. (2) 51 (1995) 342-352 MR1325577

[16] D Gilbarg, NS Trudinger, Elliptic partial differential equations of second order, second edition, Grund. der Math. Wissenschaften 224, Springer, Berlin (1983) MR737190

[17] M Gromov, Partial differential relations, Ergebnisse der Math. und ihrer Grenzgebiete (3) 9, Springer, Berlin (1986) MR864505

[18] A Haydys, Generalized Seiberg-Witten equations and hyperkähler geometry, $\mathrm{PhD}$ thesis, Universtät Göttingen (2006)

[19] A Haydys, Nonlinear Dirac operator and quaternionic analysis, Comm. Math. Phys. 281 (2008) 251-261 MR2403610

[20] P B Kronheimer, The construction of ALE spaces as hyper-Kähler quotients, J. Differential Geom. 29 (1989) 665-683 MR992334 
[21] P B Kronheimer, A Torelli-type theorem for gravitational instantons, J. Differential Geom. 29 (1989) 685-697 MR992335

[22] D McDuff, D A Salamon, J-holomorphic curves and symplectic topology, Amer. Math. Soc. Coll. Publ. 52, Amer. Math. Soc. (2004) MR2045629

[23] J D Moore, Lectures on Seiberg-Witten invariants, second edition, Lecture Notes in Math. 1629, Springer, Berlin (2001) MR1830497

[24] S Piunikhin, D A Salamon, M Schwarz, Symplectic Floer-Donaldson theory and quantum cohomology, from: "Contact and symplectic geometry (Cambridge, 1994)", (C B Thomas, editor), Publ. Newton Inst. 8, Cambridge Univ. Press (1996) 171-200 MR1432464

[25] J W Robbin, D A Salamon, The spectral flow and the Maslov index, Bull. London Math. Soc. 27 (1995) 1-33 MR1331677

[26] J W Robbin, D A Salamon, Asymptotic behaviour of holomorphic strips, Ann. Inst. H. Poincaré Anal. Non Linéaire 18 (2001) 573-612 MR1849689

[27] D A Salamon, Lectures on Floer homology, from: "Symplectic geometry and topology (Park City, UT, 1997)", (Y Eliashberg, L Traynor, editors), IAS/Park City Math. Ser. 7, Amer. Math. Soc. (1999) 143-229 MR1702944

[28] D A Salamon, E Zehnder, Morse theory for periodic solutions of Hamiltonian systems and the Maslov index, Comm. Pure Appl. Math. 45 (1992) 1303-1360 MR1181727

[29] CH Taubes, Nonlinear generalizations of a 3-manifold's Dirac operator, from: "Trends in mathematical physics (Knoxville, TN, 1998)", (V Alexiades, G Siopsis, editors), AMS/IP Stud. Adv. Math. 13, Amer. Math. Soc. (1999) 475-486 MR1708781

[30] K Wehrheim, Energy quantization and mean value inequalities for nonlinear boundary value problems, J. Eur. Math. Soc. (JEMS) 7 (2005) 305-318 MR2156603

School of Mathematical Sciences, Tel Aviv University

Ramat Aviv, Tel Aviv 69978, Israel

Mathematisches Institut, Universität Leipzig

Johannisgasse 26, 04103 Leipzig, Germany

Departement Mathematik, ETH Zentrum

Rämistrasse 101, CH-8092 Zürich, Switzerland

hohloch@post.tau.ac.il, Gregor.Noetzel@math.uni-leipzig.de, salamon@math.ethz.ch

Proposed: Simon Donaldson

Seconded: Leonid Polterovich, Yasha Eliashberg
Received: 24 October 2008

Revised: 15 April 2009 